\title{
DYNAMICS OF INELASTIC AND REACTIVE GAS-SURFACE COLLISIONS
}

\author{
LAURA ANN SMOLIAR \\ Ph.D. Thesis \\ DEPARTMENT OF CHEMISTRY \\ University of California, Berkeley \\ and \\ CHEMICAL SCIENCES DIVISION \\ Lawrence Berkeley Laboratory \\ University of California \\ Berkeley, CA 94720
}

APRIL 1995

This work was supported by the Director, Office of Energy Research, Office of Basic Energy Sciences, Materials Sciences Division, of the U.S. Department of Energy under Contract No. DE-AC03-76SF00098. 


\section{DISCLAIMER}

This report was prepared as an account of work sponsored by an agency of the United States Government. Neither the United States Government nor any agency thereof, nor any of their employees, make any warranty, express or implied, or assumes any legal liability or responsibility for the accuracy, completeness, or usefulness of any information, apparatus, product, or process disclosed, or represents that its use would not infringe privately owned rights. Reference herein to any specific commercial product, process, or service by trade name, trademark, manufacturer, or otherwise does not necessarily constitute or imply its endorsement, recommendation, or favoring by the United States Government or any agency thereof. The views and opinions of authors expressed herein do not necessarily state or reflect those of the United States Government or any agency thereof. 


\section{DISCLAIMER}

Portions of this document may be illegible in electronic image products. Images are produced from the best available original document. 
Dynamics of Inelastic and Reactive Gas-Surface Collisions

by

Laura Ann Smoliar

A.B. (Columbia University) 1990

A dissertation submitted in partial satisfaction of the requirements for the degree of

Doctor of Philosophy

in

Chemistry

in the

GRADUATE DIVISION

of the

UNIVERSITY of CALIFORNIA at BERKELEY

1995

Committee in charge:

Professor Yuan T. Lee, Chair

Professor Gabor. A. Somorjai

Professor D. R. Olander 


\author{
Abstract \\ Dynamics of Inelastic and Reactive Gas-Surface Collisions \\ by \\ Laura Ann Smoliar \\ Doctor of Philosophy in Chemistry \\ University of California at Berkeley \\ Professor Yuan T. Lee, Chair
}

The dynamics of inelastic and reactive collisions in atomic beam-surface scattering are presented. The inelastic scattering of hyperthermal rare gaseous atoms from three alkali halide surfaces was studied to understand mechanical energy transfer in unreactive systems. The dynamics of the chemical reaction in the scattering of $\mathrm{H}(\mathrm{D})$ atoms from the surfaces of $\mathrm{LiF}(001)$ and the basal plane of graphite were also studied.

First, the energy transfer from hyperthermal He atoms and $\mathrm{Ne}$ atoms to the (001) faces of $\mathrm{LiF}$ and $\mathrm{NaCl}$ single crystals was determined by measuring time-offlight spectra for the incident and scattered atomic beams. In addition, the angular dependence of scattering was explored with respect to two angles: $\theta_{i}$, the angle between the beam and the surface; and $\phi_{\mathrm{i}}$, the angle of the beam with respect to the [100] surface direction. To extend this study, the hyperthermal scattering of the series of rare gas atoms: $\mathrm{Ne}, \mathrm{Ar}, \mathrm{Kr}$, and $\mathrm{Xe}$ from a rough CsI surface is 
discussed. The experimental results are compared to the predictions of a classical impulsive collision model, where energy transfer is dependent on the masses of the collision partners and not on the collision energy.

Second, the dynamics of the formation of products in the reactions of $H(D)$ $+\operatorname{LiF}(001)$ and $\mathrm{D}+$ graphite are discussed. The last step in the reaction and subsequent desorption of the newly formed product are probed by measuring the time-of-flight spectra of the HF, DF, $C_{4}, C_{2} D_{2}$, and $C_{2} D_{4}$ product molecules as they leave the surface. There is evidence for exit channels in the potential energy surfaces of all the products observed, and in addition two types of surface reaction mechanisms are seen: Eley-Rideal and Langmuir-Hinshelwood. 
For my family. 


\section{Table of Contents}

\begin{tabular}{|c|c|c|c|c|c|c|}
\hline Abstract & - & - & - & - & - & \\
\hline Dedication & . & . & . & $\cdot$ & $\cdot$ & \\
\hline List of Figures & . & . & $\cdot$ & $\cdot$ & $\cdot$ & \\
\hline List of Tables & - & . & . & . & - & \\
\hline Acknowledgements & - & • & . & - & * & \\
\hline Chapter 1 & - & $\cdot$ & $\cdot$ & ${ }^{\circ}$ & $\cdot$ & \\
\hline
\end{tabular}

Chapter 2 Scattering of Hyperthermal Rare Gas Beams from Two Alkali

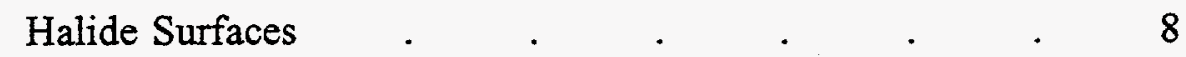

Introduction . $\quad . \quad . \quad . \quad . \quad . \quad . \quad 8$

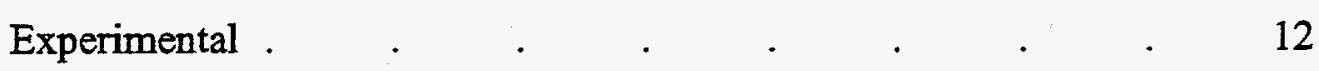

Results . $\quad . \quad . \quad . \quad . \quad . \quad . \quad . \quad 14$

Scattering from $\operatorname{LiF}(001) \quad$. $\quad . \quad$. $\quad . \quad$. 15

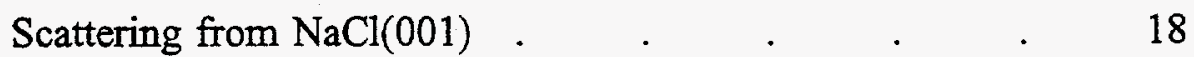

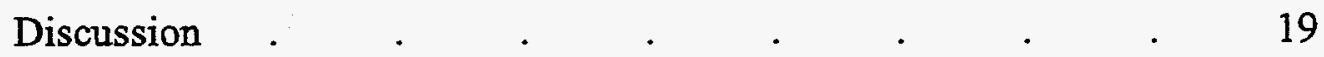

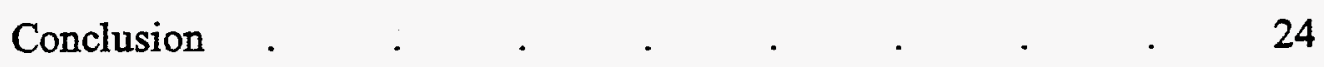

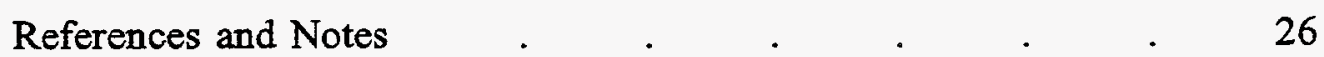

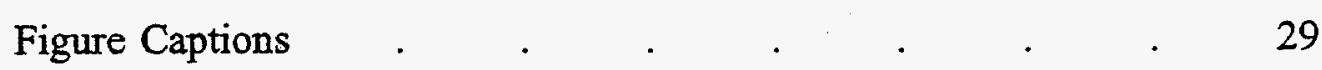


Figures

Chapter 3 Energy Transfer in the Scattering of Hyperthermal Rare Gas

Beams from a CsI Surface . $\quad . \quad \ldots \quad$. $\quad . \quad 4 \quad 49$

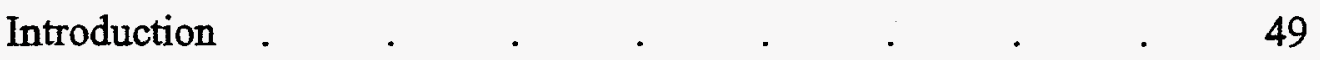

Experimental . $\quad . \quad \ldots \quad \ldots \quad$. $\quad . \quad \ldots \quad$. 51

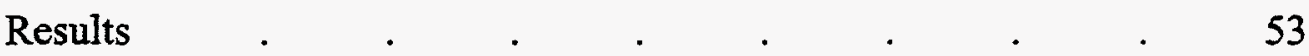

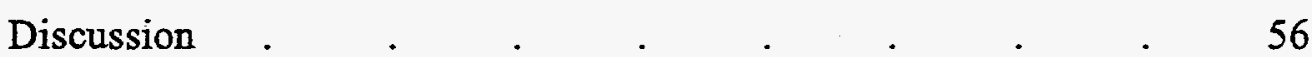

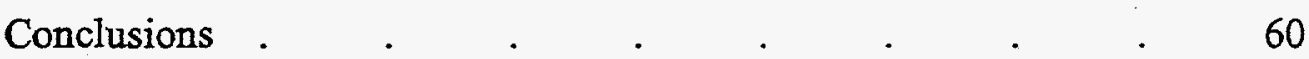

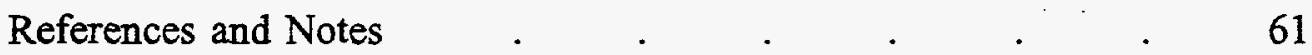

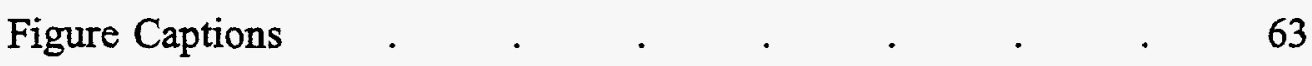

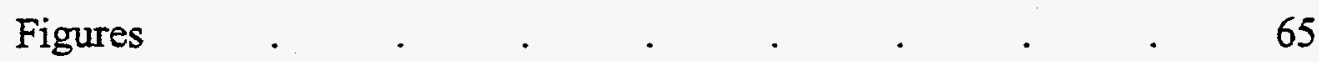

Chapter 4 Atomic Beam-Surface Scattering Study of HF(DF) Produced in the Reaction: $\mathrm{H}(\mathrm{D})+\operatorname{LiF}(001)$. . . . . $\quad 72$

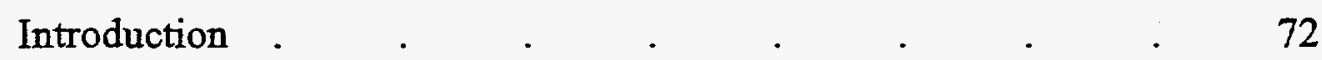

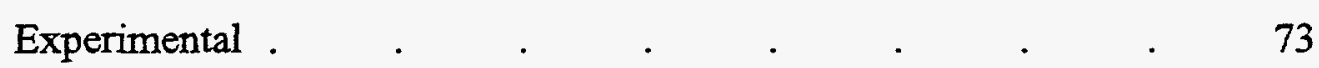

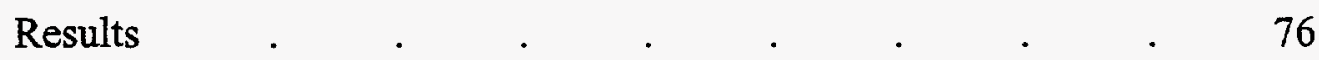

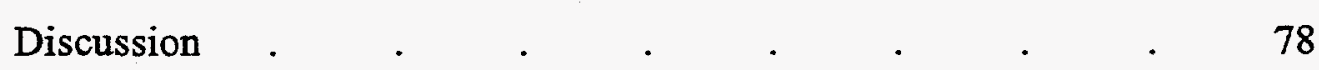

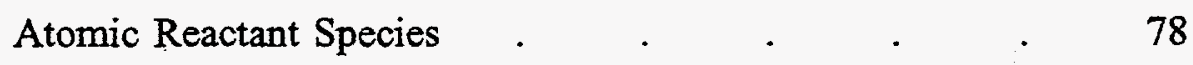

Origin of the Fast Time-of-Flight Peak . . . . 79

Dynamics of HF and DF Products . $\quad . \quad \ldots \quad$. $\quad . \quad 82$

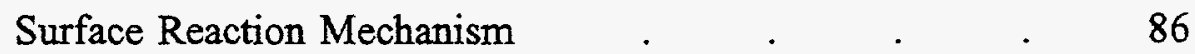




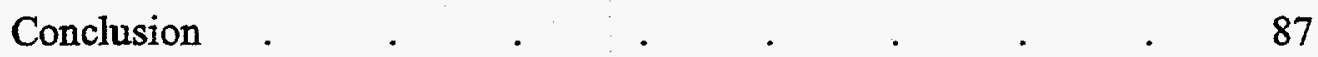

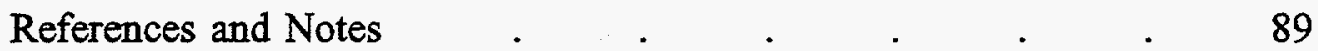

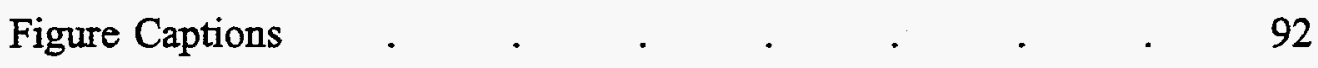

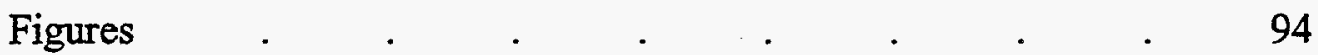

Chapter 5 Dynamics of the Acetylene- $\mathrm{d}_{2}$, Ethylene- $\mathrm{d}_{4}$, and

Tetradeuteromethane Products from the Reaction

of D-Atoms with a Graphite Surface _ . . . . 103

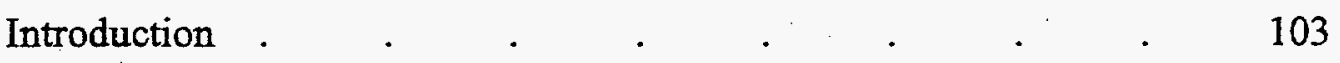

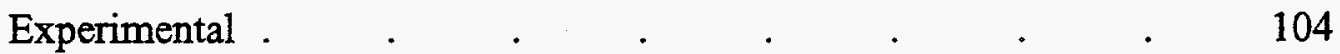

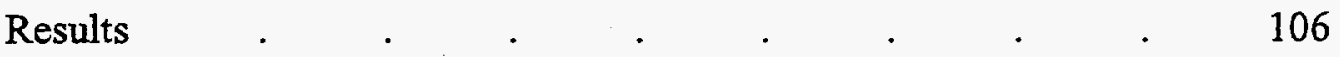

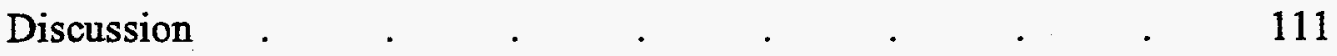

Product Translational Energy $\quad . \quad \ldots \quad$. $\quad . \quad$. $\quad 111$

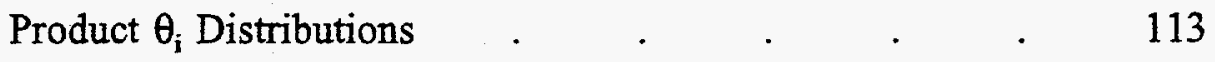

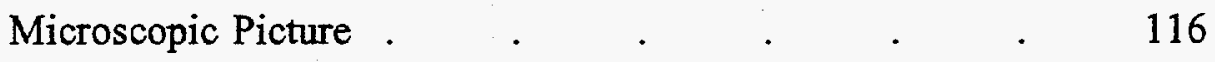

Comparison with other Molecular Beam Etching Studies . 118

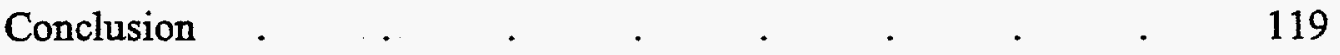

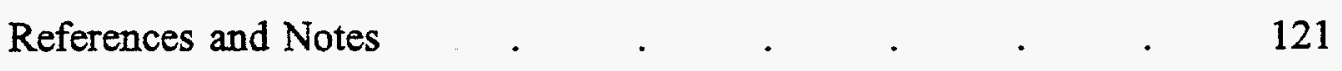

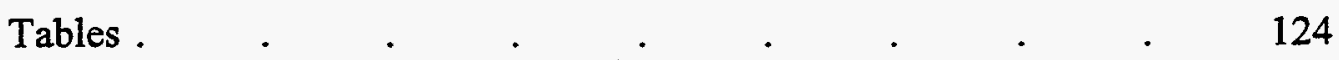

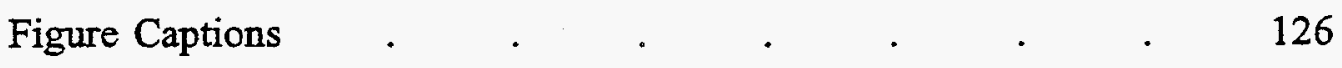

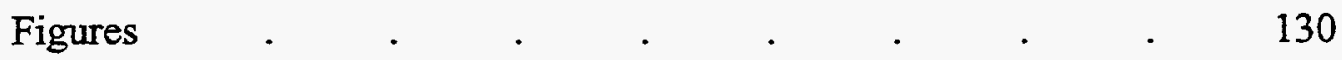




\section{$\underline{\text { List of Figures }}$}

Fig. 2-1 Gas-Surface Scattering Apparatus . . . . . 34

Fig. 2-2 Beam Characterization for $0.6 \% \mathrm{Ne} / \mathrm{H}_{2}$ and $1 \% \mathrm{He} / \mathrm{H}_{2} \quad$. 35

Fig. 2-3 Time-of-Flight Data from 3 and $20 \mathrm{kcal} / \mathrm{mole}$ He Atoms

Scattered from $\operatorname{LiF}(001) \quad$. $\quad . \quad$. $\quad$. $\quad$. 36

Fig. 2-4 Translational Energy Distributions for the

Fits in Fig. 2-3 $\quad$. $\quad . \quad$. $\quad$. $\quad$. $\quad$. 37

Fig. 2-5 Energy Transfer as a Function of Collision Energy - 38

Fig. 2-6 The $\phi_{i}$ Dependence of He Atom Scattering

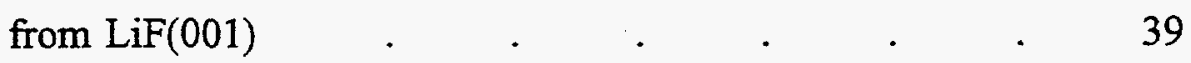

Fig. 2-7 Ne Atoms Scattered from $\operatorname{LiF}(001)$ with

$\mathrm{E}_{\mathrm{coll}}=45 \mathrm{kcal} / \mathrm{mole} \quad . \quad . \quad . \quad . \quad . \quad . \quad .40$

Fig. 2-8 The $\phi_{i}$ Dependence of Ne Atom Scattering

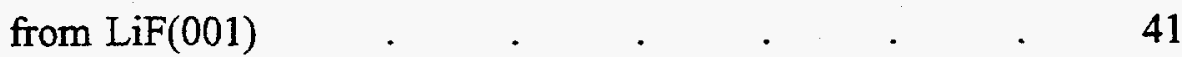

Fig. 2-9 Time-of-Flight data from 3 and $20 \mathrm{kcal} / \mathrm{mole}$ He Atoms

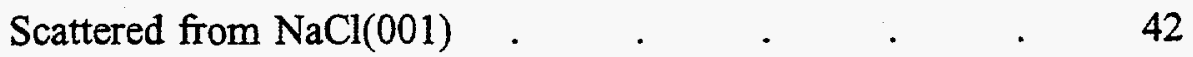

Fig. 2-10 Translational Energy Distributions for the

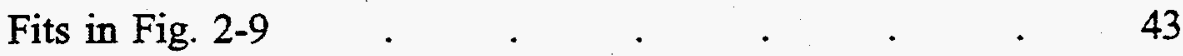

Fig. 2-11 Ne Atoms. Scattered from $\mathrm{NaCl}(001)$ with

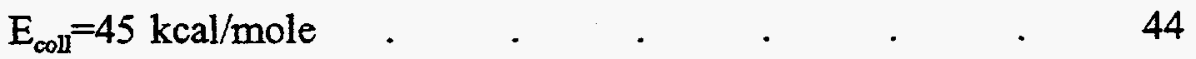

Fig. 2-12 The $\phi_{i}$ Dependence of Ne Atom Scattering 


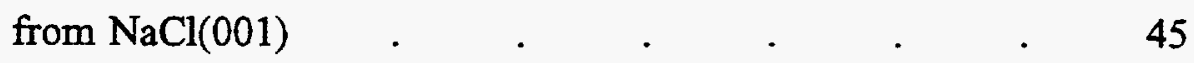

Fig. 2-13 $\theta_{\mathrm{i}}$ Distribution for $13 \mathrm{kcal} / \mathrm{mole} \mathrm{Ne}$ Atoms

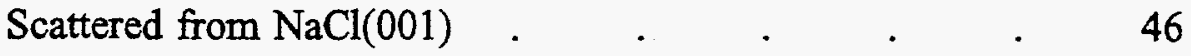

Fig. 2-14 Relative Ion Sizes in $\mathrm{LiF}(001)$ and $\mathrm{NaCl}(001) \quad$. $\quad 47$

Fig. 2-15 Three Types of Scattering, $\theta_{\mathrm{i}}$ Dependence . $\quad . \quad$. $\quad 48$

Fig. 3-1 Beam Characterization $\quad . \quad$. $\quad . \quad . \quad . \quad 65$

Fig. 3-2 Time-of-Flight Data for $\mathrm{Ne}, \mathrm{Ar}, \mathrm{Kr}$, and $\mathrm{Xe}$ Atoms Scattered from CsI with $\mathrm{E}_{\text {coll }}=20 \mathrm{kcal} / \mathrm{mole} \quad$. $\quad . \quad . \quad . \quad 66$

Fig. 3-3 Time-of-Flight Data for Ar Atoms with

Three Incident Energies _ $\quad \ldots \quad$. $\quad . \quad 67$

Fig. 3-4 Time-of-Flight Data for Scattered $\mathrm{Kr}$ Atoms with

$E_{\text {coll }}=50 \mathrm{kcal} / \mathrm{mole}$ and $\mathrm{Xe}$ Atoms with $E_{\text {coll }}=65 \mathrm{kcal} / \mathrm{mole}$. $\quad 68$

Fig. 3-5 Translational Energy Distribution for Scattered $\mathrm{Kr}$ Atoms

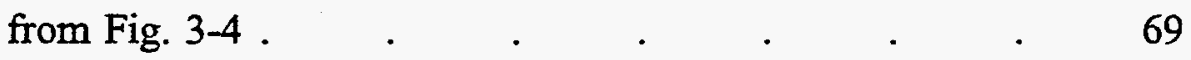

Fig. 3-6 Translational Energy Distribution for Scattered Xe Atoms from Fig. 3-4 . $\quad$. $\quad . \quad$. $\quad . \quad$. 70

Fig. 3-7 Energy Transfer as a Function of Collision Energy $\quad$ - 71

Fig. 4-1 Nozzle Temperature as a Function of Power . . $\quad 94$

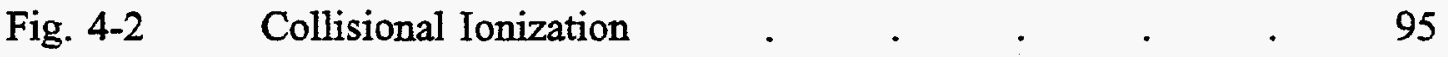

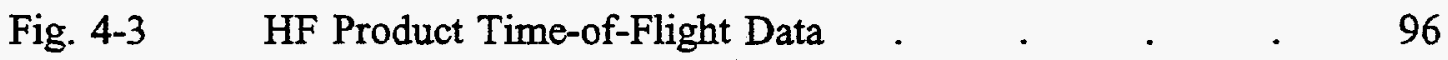

Fig. 4-4 DF Product Time-of-Flight Data $\quad . \quad$. $\quad . \quad . \quad 97$

Fig. 4-5 HF and DF Translational Energy Distribution _ . $\quad . \quad 98$ 
Fig. 4-6 $\quad \theta_{i}$ Distributions for HF and DF $\quad . \quad \ldots \quad . \quad . \quad . \quad 99$

Fig. 4-7 Evidence for Exit Channel Barrier . . . . . 100

Fig. 4-8 Microscopic Picture . $\quad . \quad \ldots \quad$. $\quad . \quad \ldots \quad . \quad 101$

Fig. 4-9 Polar Plot of $\theta_{i}$ Distribution for HF . $\quad$. $\quad . \quad$. 102

Fig. 5-1 Time-of-Flight Data for $\mathrm{m} / \mathrm{e}=18,20 \quad$. $\quad . \quad . \quad 130$

Fig. 5-2 Tetradeuteromethane Translational Energy

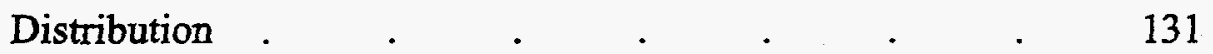

Fig. 5-3 Time-of-Flight Data for $\mathrm{m} / \mathrm{e}=20$ at Different $\theta_{\mathrm{i}} \quad$. $\quad . \quad 132$

Fig. 5-4 Time-of-Flight Data for $\mathrm{m} / \mathrm{e}=32$ at Different $\theta_{i} \quad . \quad$. 133

Fig. 5-5 Ethylene-d $d_{4}$ Translational Energy Distribution $\quad . \quad$. $\quad 134$

Fig. 5-6 Time-of-Flight Data for $m / e=26$ at Different $\theta_{i} \ldots \quad$. $\quad 135$

Fig. 5-7 Acetylene- $\mathrm{d}_{2}$ Translational Energy Distribution . . 136

Fig. 5-8 Time-of-Flight Data for $\mathrm{m} / \mathrm{e}=16$ at Different $\theta_{\mathrm{i}} \quad . \quad$. $\quad 137$

Fig. 5-9 Time-of-Flight Data for $\mathrm{m} / \mathrm{e}=14 \quad \ldots \quad \ldots \quad \ldots \quad \ldots$

Fig. 5-10 Time-of-Flight Data for $\mathrm{m} / \mathrm{e}=30 \quad \ldots \quad \ldots \quad \ldots \quad \ldots$

Fig. 5-11 Evidence for Exit Channel Barriers . $\quad . \quad$. $\quad . \quad 140$

Fig. 5-12 Polar Plot of $\theta_{i}$ Distribution for Acetylene- $d_{2} \quad \ldots \quad$. $\quad 141$

Fig. 5-13 Polar Plot of $\theta_{\mathrm{i}}$ Distribution for Ethylene-d $\mathrm{d}_{4} \quad \ldots \quad$. $\quad 142$

Fig. 5-14 Polar Plot of $\theta_{\mathrm{i}}$ Distribution for

Tetradeuteromethane . $\quad . \quad . \quad . \quad . \quad . \quad . \quad 143$ 


\section{List of Tables}

\section{Chapter 5}

Table I Assignment of Detected Ions from Reaction _ . . $\quad 124$

$\begin{array}{lllllllllll}\text { Table II } & \text { Fragmentation Patterns } & & & & & & & & & \end{array}$ 


\section{Acknowledgements}

Professor Yuan T. Lee has truly been an inspiration to work for, and I will never be able to thank him enough for the many unusual opportunities that he gave me in addition to wonderful scientific guidance. The most exciting of these was spending seven months in Taipei at the Institute of Atomic and Molecular Sciences, Academia Sinica, where I wrote this thesis. I also appreciated the copious advice that rolled out of the FAX machine when I was really stuck, especially during the time I was trying to assemble the Gas Surface Machine (GSM). Yuan always supported me through the tough times at Berkeley, and taught me a lot about people in the process.

When I started in the Lee Group, Dr. Deon Anex, David Gosalvez, and Mathew Lewin were my co-workers in $\mathrm{D} 43$, and they introduced me to many resources at Berkeley. In D43, I also had the pleasure of working with Nancy Chu, Cindy Berrie, and Dr. Gang-Yu Liu.

Almost everyone in the group has made a contribution to the running of the GSM. Floyd Davis was full of great advice about detectors and beams and had the idea of using $\mathrm{Cl}_{2}$ and $\mathrm{Br}_{2}$ photodissociation to calibrate the detector. He also had the great talent of making everything look easy and was always happy to talk about experiments. Jim Myers taught me about interfacing and programming in $\mathrm{C}++$ and was instrumental in getting the data acquisition working. We had many useful discussions about ways to approach data fitting. Tzong-Tsong Miau helped me 
with calibrating the rhenium nozzle on the $35^{\prime \prime}$ machine, brought me food when I was running the experiment for long periods of time, and would stay up late to help me fix broken equipment. Miau is a master of machines and what I know about building, fixing, and maintaining the GSM I learned from him. Arthur Suits was instrumental in convincing me to NOT use an LSI-11 computer to take data, for which I am forever grateful. It was nice to have him return recently since he is always happy to discuss data (and I finally had some to discuss when he got back).

I enjoyed late night discussions about science with my officemates, Allan Bracker and James Chesko, who could always make me laugh. Dr. Kim Prather was my colleague and roommate during my most difficult time in graduate school. She was extremely supportive during my second year orals. Thanks also to Jingsong Zhang, Doo Wan Boo, Hongtao Hou, and Marcus J. J. Vrakking for their help and equipment loans. Ann Lawhead helped me get through all the bureaucracy of LBL and Berkeley, and is always a source of useful information. Thanks to the machinists who did an excellent job on the GSM parts, Bob Conroy of the LBL machine shop who welded the rhenium nozzles, and the electronics and glass shops.

In preparation for Taiwan, Xueming Yang was helpful to consult about mechanical drawing and tolerances on the rotating source machine. Zhifeng Liu helped me master data transfer over the Internet to Taiwan and he, Miau, Jinchun Xie and K. T. Liu attempted to teach me some Mandarin.

My experience in Taiwan was wonderful because of the many generous 
people at the Institute of Atomic and Molecular Sciences (IAMS) that made us feel at home. Professor Shen-maw Lin let me monopolize his best computer to write this thesis, and I enjoyed my daily discussions with him about science, Chinese food, Taiwan, and Berkeley in the 1960's. Dr. Y. R. Lee was a tremendous help with experiments in Taiwan and knew all the best places to eat around the National Taiwan University. Tiffany Yeh helped us with experiments and all kinds of daily tasks that I thought were trivial until I tried to do them in Mandarin. The surface group at IAMS was great to talk to. In particular I would like to thank Dr. T. J. Chuang and Dr. J. C. Lin for the helpful ideas that came out of our discussions. Cheryl Longfellow was my constant companion in and out of the lab, and I want to thank her for asking me to go to Taiwan with her and teaching me interesting things about IRMPD and photodissociation in general. Mrs. Hsui-Chuan Shaw made sure that we always saw Yuan when he came to visit IAMS, and helped us track him down in different parts of the world when necessary.

While in Asia, I had the opportunity to visit research institutes in China and Japan. I would like to thank Professor Fanao Kong for hosting us in Beijing and Professor Toshinori Suzuki for inviting me to the Institute for Molecular Science in Okazaki, where I had many helpful discussions about gas-surface dynamics. I have enjoyed my collaboration with Dr. Robert Sinkovits, from whom I learned about molecular dynamics simulations. I would like to thank Dr. Mark Cardillo, Dr. John Tully, and Dr. Alex Harris of AT\&T Bell Laboratories for support and guidance throughout my last 5 years. I found it particularly helpful to 
talk to them when deciding what experiments would be feasible on the GSM, and Dr. Cardillo's visits to Berkeley always gave me a boost of confidence when I needed it most. I would also like to thank AT\&T Bell Laboratories for a Ph.D. Scholarship.

I was first introduced to the field of surface chemistry in Professor Brian Bent's lab at Columbia University. Brian taught me everything from tightening conflat bolts and swagelocks to how to plan a good experiment and think about data. Everything I learned from Brian and his two senior graduate students, Cindy Jenks and Chao-Ming Chiang, was immensely useful in graduate school. Professor George Flynn inspired me to change my major from math to chemistry in college and continues to be full of great "free advice". He has also been a great role model, and I have tried to emulate him when teaching at Berkeley. Hannah Sevian was first my physical chemistry TA and then one of my closest friends and has always given me a good perspective on life in graduate school at Berkeley.

During my last years in Berkeley, Nerine Cherepy and Erica Kuo gave me something to look forward to when coming home from the lab. I also enjoyed our lunches with Marcia Ziegeweid, who was always full of information about postdocs and jobs as well as funny stories.

I owe my education to my family. My Mother first introduced me to fun toys in her lab when I was a child, and I want her to know that all those late nights she spent teaching me physics and chemistry have indeed paid off! My Father taught me math and writing and kept me from giving up at times when my morale 
was low. My Uncle, Gerald Smoliar, always kept up with my research and was the first person to teach me about computers.

This project was generously supported by the Office of Naval Research through Dr. Richard Miller. 


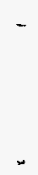




\section{Chapter 1}

\section{Introduction}

The dynamics of collisions between gaseous molecules and a solid surface are of fundamental importance for understanding gas-surface reactions. ${ }^{1}$ The work presented here explores gas-surface interactions at the molecular level, both unreactive and reactive, with a focus on the role of energy transfer between the gas and the surface, energy partitioning in reaction products in the case of reactive collisions, and a mechanistic picture of the events taking place on the surface.

The use of supersonic atomic or molecular beams to study gas-surface

dynamics has been well established. ${ }^{2,3}$ The supersonic beam introduces gaseous atoms or molecules into the collision zone with a well defined velocity. In the hyperthermal scattering of rare gaseous atoms of Chapters 2 and 3 , the incident energy of the atoms is well controlled by using the seeded beam technique and adjusting the temperature of a resistively heated rhenium nozzle. ${ }^{4}$ In addition, beams of reactive radical species can be introduced with well-defined incident energies. Reactive $\mathrm{H}$ and $\mathrm{D}$ atoms are used in the scattering experiments described in Chapters 4 and 5.

We built a machine called the "Gas-Surface Machine" (GSM) to do the experiments presented in this thesis. The machine, which is described in detail in 
Chapter 2, allows for direct velocity measurement of particles as they evolve from the surface without undergoing any other collisions. The mass of the particles is also known, and so the translational energy is obtained. In the case of reactions, the translational energy distributions of individual reaction products from a complex system can be measured individually (Chapter 5). The translational energy distribution of the products reflects the dynamics of the last step in the gas-surface interaction. In the case of a inelastic scattering, the energy lost to the surface from different types of collisions is obtained. In the case of reactions, important features of the exit channel of the potential energy surface are revealed, such as whether there is a barrier and how energy is partitioned. The geometry of the GSM is such that the angle between the incident beam and the detector axis is always fixed at $90^{\circ}$. The angles $\theta_{\mathrm{i}}$, between the incident beam and the surface plane, and $\phi_{\mathrm{i}}$, the angle with respect to a direction along the lattice of the crystal (in the case where it is well defined, i.e. the [100] direction in $\operatorname{LiF}(001))$, were varied so that $\theta_{i}+\theta_{s}=$ $90^{\circ}$ and $\phi_{s}=\phi_{i}+180^{\circ}$. The change in scattered product flux with these angles gives a better understanding of the mechanisms for either energy loss (Chapters 2 and 3 ) or product formation (Chapters 4 and 5) occurring at the surface.

The ultimate goal we strived to achieve was to learn about the dynamics of individual reaction products from a gas-surface reaction. However, as we were working with a new machine and also wanted to understand the role of energy transfer in gas-surface collisions, we decided to start with a less complex system. Thus, we chose to start with hyperthermal scattering of rare gaseous atoms from 
various alkali halide surfaces: $\mathrm{LiF}(001), \mathrm{NaCl}(001)$, and $\mathrm{CsI}$ (rough). The rationale was that an understanding of energy transfer in collisions of unreactive systems would be important for later sorting out chemical and non-chemical effects in more complex reactive systems. In other words, we wanted to be able to later distinguish true chemical effects from purely mechanical ones when looking at the different features in a measured time-of-flight spectrum. As it turned out, the reactive systems studied involved $\mathrm{H}$ and $\mathrm{D}$ atoms, which are quite light, and so the mechanical effects are minimal. However, it is anticipated that if scattering with heavier atoms such as $\mathrm{Cl}$ or other halogens is explored in the future, mechanical energy transfer will be an important aspect of the observed dynamics when the collision energy is in the hyperthermal regime.

Chapter 2 describes the hyperthermal scattering of $\mathrm{He}$ atoms and $\mathrm{Ne}$ atoms from $\mathrm{LiF}(001)$ and $\mathrm{NaCl}(001)$. Time-of-flight spectra are measured for the atoms as they leave the crystal surface, and translational energy distributions are obtained that reflect how much energy the atom has left over after the collision event at the surface. The series of rare gas atoms: $\mathrm{Ne}, \mathrm{Ar}, \mathrm{Kr}$, and $\mathrm{Xe}$ was used for hyperthermal scattering off a rough surface of CsI, and these experiments are reported in Chapter 3. In both these experiments, the ideas behind the binary collision models often used to describe classical collisions are tested. ${ }^{1,5}$ In particular, the idea that the energy transfer in the collision is solely dependent on the masses of the collision partners and not the incident energy can be well tested by looking at a range of rare gases on different alkali halide surfaces. 
The real goal of this project was to study reactive systems, and so the second half of this thesis describes the reaction dynamics of $\mathrm{H}$ or $\mathrm{D}$ atoms scattered from two different surfaces, $\operatorname{LiF}(001)$ and the basal plane of HOPG graphite. The kinetics of many gas-surface reactions have been studied effectively using molecular beams, ${ }^{6-12}$ but the dynamics have not been as frequently explored. The dynamics of some reactions important to catalysis, such as the oxidation of $\mathrm{CO}$ on platinum and the oxidation of deuterium on platinum have been studied. ${ }^{13-14}$ However, the reactions reported in Chapters 4 and 5 are etching reactions, where the substrate itself is one of the reactants. The dynamics of etching reactions have not been studied extensively. F. A. Houle has studied the dynamics of silicon etching by $\mathrm{XeF}_{2}$, but in that case the reactant is introduced using an effusive beam and modulated beam techniques are used to monitor product evolution. ${ }^{15}$ The dynamics of etching products from $\mathrm{Cl}_{2}$ etching of $\mathrm{GaAs}(110),{ }^{16} \mathrm{Cl}_{2}$ etching of $\mathrm{Si}(111),{ }^{17}$ and $\mathrm{Cl}_{2}$ etching of $\mathrm{GaAs}(100){ }^{18}$ have been reported using similar time-offlight techniques to what is used in this thesis.

In Chapter 4, the reaction of $\mathrm{H}(\mathrm{D})+\mathrm{LiF}(001)$ is studied by measuring the time-of-flight of the HF or DF product of the gas-surface reaction. In addition to learning about the exit channel of the potential energy surface for this reaction, which has a barrier, the mechanism of the reaction is determined to be of the EleyRideal type. The Eley-Rideal mechanism refers to reactions that occur between a gaseous atom and a surface atom or surface adsorbate. The other common mechanism, more commonly reported for catalysis reactions, is the Langmuir- 
Hinshelwood mechanism, which is a reaction between two adsorbed species on the surface to form a product that is then released into the gas phase. ${ }^{19,20}$

In Chapter 5 , the reaction of $\mathrm{D}$ atoms with a graphite surface, producing $\mathrm{CD}_{4}, \mathrm{C}_{2} \mathrm{D}_{2}$, and $\mathrm{C}_{2} \mathrm{D}_{4}$ is reported. $\mathrm{C}_{2} \mathrm{H}_{4}$ was not seen previously in modulated molecular beam work of the $\mathrm{H}+$ graphite reaction when an effusive source of $\mathrm{H}$ atoms was used. ${ }^{11}$ Here the D-atoms, produced in a supersonic beam, have an incident kinetic energy of $\sim 7.5 \mathrm{kcal} /$ mole and the flux is two orders of magnitude higher. The translational energy distributions for all three products are obtained, giving information about the potential energy surfaces involved in the three reactions. In addition, evidence for both the Eley-Rideal and LangmuirHinshelwood mechanisms is presented. In the case of $\mathrm{CD}_{4}$ formation, there is evidence that both mechanisms operate simultaneously, while for $C_{2} D_{2}$ and $C_{2} D_{4}$, the Langmuir-Hinshelwood mechanism governs the reaction. 


\section{References}

1. C. T. Rettner and M. N. R. Ashfold (Ed.), Dynamics of Gas-Surface Interactions (The Royal Society of Chemistry, London, 1991).

2. J. A. Barker and D. J. Auerbach, Surf. Sci. Rep. 4, 1 (1984).

3. S. T. Ceyer, D. G. Gladstone, M. McGonigal, and M. T. Schulberg, Investigations of Surfaces and Interfaces-PartA (John Wiley and Sons, Inc., New York, 1993), pp. 383-452.

4. Details of the nozzle construction are presented in Chapter 4.

5. F. O. Goodman and H. Y. Wachmann, Dynamics of Gas-Surface Scattering (Academic Press, New York, 1976).

6. C. Su, Z. G. Dai, W. L. Luo, D. H. Sun, M. F. Vernon, and B. E. Bent, Surf. Sci. 312, 181 (1994).

7. C. Su, H. Q. Hou, G. H. Lee, Z. G. Dai, W. Luo, M. F. Vernon, and B.E. Bent, J. Vac. Sci. Technol. B 11, 1222 (1993).

8. C. Su, M. Xi, Z. G Dai, M. F. Vernon, and B. E. Bent, Surf. Sci. 282, 357 (1993).

9. D. R. Olander, W. Siekhaus, R. Jones, and J. A. Schwarz, J. Chem. Phys. 57, 408 (1972).

10. D. R. Olander, R. H. Jones, J. A. Schwarz, and W. J. Siekhaus, J. Chem. Phys. 57, 421 (1972). 
11. M. Balooch and D. R. Olander, J. Chem. Phys. 63, 4772 (1975).

12. M. J. Vasile and F. A. Stevie, J. Appl. Phys. 53, 3799 (1982).

13. C. A. Becker, J. P. Cowin, L. Wharton, and D. J. Auerbach, J. Chem. Phys. 67, 3394 (1977).

14. S. T. Ceyer, W. L. Guthrie, T.-H. Lin, and G. A. Somorjai, J. Chem. Phys. 78, 6982 (1983).

15. F. A. Houle, J. Chem. Phys. 87, 1866 (1987).

16. L. A. DeLouise, J. Chem. Phys. 94, 1528 (1991).

17. H. Yoshikawa and K. Shobatake, Chem. Phys. Lett. 223, 341 (1994).

18. P. Bond, P. N. Brier, J. Fletcher, P. A. Gorry, and M. E. Pemble, Chem. Phys. Lett. 208, 269 (1993).

19. J. I. Steinfeld, J. S. Francisco, and W. L. Hase, Chemical Kinetics and Dynamics (Prentice Hall International, Inc., London, 1989) p.196.

20. M. L. Yu and L. A. DeLouise, Surf. Sci. Rep. 19, 285 (1994). 


\section{Chapter 2}

\section{Scattering of Hyperthermal Rare Gas Beams from Two Alkali Halide Surfaces}

\section{Introduction}

We have investigated the energy transfer in collisions between hyperthermal rare gas atoms and the surfaces of alkali halide crystals using atomic beam surface scattering. In this chapter, the experimental results for the following four systems are addressed: $\mathrm{He}-\mathrm{LiF}(001), \mathrm{Ne}-\mathrm{LiF}(001), \mathrm{He}-\mathrm{NaCl}(001)$, and $\mathrm{Ne}-\mathrm{NaCl}(001)$. He atoms, with collision energies in the range $\left[3<\mathrm{E}_{i}(\mathrm{kcal} / \mathrm{mole})<20\right]$, and Ne atoms, with collision energies in the range $\left[13<\mathrm{E}_{\mathrm{i}}(\mathrm{kcal} / \mathrm{mole})<66\right]$, were incident on each surface, and the time-of-flight spectra of the scattered atoms were recorded under single-collision conditions. The angles $\theta_{\mathrm{i}}$ between the incident beam and the surface plane, and $\phi_{\mathrm{i}}$ the angle with respect to the [100] surface direction, were varied with the detector fixed at $90^{\circ}$ from the beam so that $\theta_{i}+\theta_{s}=90^{\circ}$ and $\phi_{s}=$ $\phi_{\mathrm{i}}+180^{\circ}$. By measuring the time-of-flight spectra of the recoiling atoms for a given incident beam energy, details about the dynamics and the energy transfer in the atom-surface collision are obtained.

Gas phase investigations of collisions between rare gas atoms and alkali halide molecules showed that the energy transfer could be distinguished for two different near collinear configurations and modelled by the impulse approximation. ${ }^{1}$ 
An important feature of the impulse approximation for the fractional energy transfer, $\Delta \mathrm{E} / \mathrm{E}$, is the dependence on the masses of the colliding species and the independence of collision energy:

$$
\frac{\Delta E}{E}=\frac{4 M_{a} M_{b} M_{c}\left(M_{a}+M_{b}+M_{c}\right)}{\left(M_{a}+M_{b}\right)^{2}\left(M_{b}+M_{c}\right)^{2}} \leq 1,
$$

where $M_{a}$ is the mass of the free atom, $M_{b}$ is the mass of the target atom in the diatomic molecule, and $M_{c}$ is the mass of the second atom in the diatomic molecule. For example, the energy transfer in collisions between $\mathrm{Xe}$ and $\mathrm{CsBr}$ in the following two collinear configurations was quite different:

$\mathrm{Xe}+\mathrm{BrCs}$,

$X e+C s B r$

The experimental results showed $95 \%$ energy transfer for (2) and $61 \%$ energy transfer for (3), and predictions based on the impulse approximation were $95.0 \%$ for (2) and $60.7 \%$ for (3). ${ }^{2}$

To predict the energy transfer in the case of a free atom $\left(\mathrm{M}_{\mathrm{a}}\right)$ hitting a surface atom $\left(M_{b}\right)$, we let $M_{c} \rightarrow \infty$. The equation for energy transfer then becomes: 
and is valid when $M_{a} \leq M_{b}{ }^{3,4}$ We have studied the scattering of He atoms and Ne

$$
\frac{\Delta E}{E}=\frac{4 M_{a} M_{b}}{\left(M_{a}+M_{b}\right)^{2}} \leq 1,
$$

atoms from $\mathrm{LiF}(001)$ and $\mathrm{NaCl}(001)$ surfaces to determine if 1) the energy transfer is dominated by the impulsive collisions between the rare gas atoms and individual atoms comprising the alkali halide surface, 2) $\Delta \mathrm{E} / \mathrm{E}$ is independent of collision energy as predicted by this simple model, and 3) the model predicts the energy transfer from rare gas atoms of different masses to alkali halide salts containing different masses for the anion and cation. It is our hope that a full understanding of energy transfer in the hyperthermal regime of these non-reactive systems will help in future studies of reactive systems where chemical effects and non-chemical effects both play a role in the dynamics of the gas-surface interaction. ${ }^{5}$

While there is a wealth of data on the scattering of light rare gases, most frequently $\mathrm{He}$ atoms, from the surfaces of $\mathrm{LiF}$ and $\mathrm{NaCl}^{6}$, at low collision energies (on the order of several $\mathrm{meV}$ ), the scattering in the energy range of up to $3 \mathrm{eV}$ has not been widely studied. Low collision energies were used to study diffraction, elastic, and inelastic scattering from these surfaces. The goal of these low energy inelastic scattering experiments was to understand the energy transfer to and from single phonon modes, and great pains were taken to minimize multi-phonon 
scattering. ${ }^{7}$ Our experiments, on the other hand, are in an energy regime where highly inelastic scattering events, involving the creation of a large number of phonons, can be studied. Such hyperthermal scattering has been studied for Xe scattered from the surfaces of $\operatorname{GaAs}(110), \mathrm{Ag}(100)$, and $\mathrm{Ge}(100),{ }^{4}$ but there is no systematic study of the alkali halide salts. Saecker and Nathanson ${ }^{8}$ have investigated the scattering of $\mathrm{Ne}, \mathrm{CH}_{4}, \mathrm{NH}_{3}$, and $\mathrm{D}_{2} \mathrm{O}$ with surfaces of perfluorinatedpolyether, glycerol, and squalane using similar beam-surface scattering experiments. These studies of atomic and molecular collisions with liquid surfaces show impulsive collision behavior and give insight into what functional groups the incoming gas particles see in collisions with the liquid surface.

In the experiments described in this chapter, the mechanisms of energy transfer from the incoming rare gas atom to the alkali halide surface are described. The time-of-flight spectrum of the recoiling atom reflects the amount of energy the particles retain after collision, and since the incident energy of the atomic beam is known, the energy transfer to the surface is easily calculated. The features in the time-of-flight spectrum reveal what types of energy loss occurred at the surface. It will be shown, for example, that $\mathrm{Ne}$ atom collisions with each surface show two distinct features whereas He atom scattering shows only one. From the large amounts of energy transferred to $\mathrm{LiF}(001)$ and $\mathrm{NaCl}(001)$, especially in the case of Ne atom scattering, it can be concluded that these crystals are not behaving as rigid walls. Rather, such highly inelastic collisions suggest that the crystal is 
flexible, which is not surprising if many phonon modes are excited in the energy transfer process.

\section{Experimental}

The atomic beam surface scattering apparatus, shown schematically in Fig. 1, was used for the experiment. A continuous supersonic beam of rare gas atoms was produced and their time-of-flight after scattering from the surface of an alkali halide single crystal mounted on a three-axis rotatable manipulator was measured. The source-detector angle is fixed at $90^{\circ}$, and the crystal can be rotated to change the incident angle between the beam and the surface, $\theta_{i}$, as well as the orientation of the [100] direction of the crystal, $\phi_{i}$.

A resistively heated rhenium nozzle with a .007 " diameter hole, described elsewhere, ${ }^{9}$ is used to generate a supersonic beam of rare gas mixtures. Seeded beams were made with $1 \% \mathrm{He}$ in $\mathrm{H}_{2}$ and $0.6 \% \mathrm{Ne}$ in $\mathrm{H}_{2}$. The beam velocity and speed ratio were characterized for each mixture as a function of 1) stagnation pressure, and 2) the power used to heat the nozzle, on a crossed molecular beams apparatus by measuring beam time-of-flight using a spinning slotted wheel chopper and with the detector looking directly into the beam. ${ }^{10}$ The time-of-flight data were fit to the following equation, which is appropriate for a supersonic beam:

$$
N(v)=v^{2} \exp \left(-\beta\left(v-v_{0}\right)^{2}\right),
$$

where $N(v)$ is a number density distribution of speeds; $\beta=m / 2 k T_{s}$, in $(\mathrm{m} / \mathrm{s})^{-2}$, 
characterizes the width of the distribution (where $T_{s}$ is the "translational temperature" of the beam); and $\mathrm{v}_{0}$ is the most probable velocity in the distribution, $N(v)$. The beam energy is calculated from $v_{0}$. The speed ratio, $S=v_{0}(\beta)^{1 / 2}>1$, characterizes the quality of the supersonic expansion. ${ }^{11}$ The nozzle temperature was determined by measuring beam time-of-flight for pure Ne and also using optical pyrometry. The results of the characterization using beam time-of-flight measurements are shown in Fig. 2. The stagnation pressure was adjusted to create a good expansion as the temperature of the nozzle was increased. Speed ratios of 5-8 and 6-11 were obtained for the $1 \% \mathrm{He} / \mathrm{H}_{2}$ and $0.6 \% \mathrm{Ne} / \mathrm{H}_{2}$ mixtures, respectively.

The atomic beam source is differentially pumped by a $6,600 \mathrm{l} / \mathrm{s}$ diffusion pump backed by a $169 \mathrm{cfm}$ mechanical booster pump (Edwards EH250F/E2M40F) in the source region, ${ }^{12}$ which contains a $.017^{\prime \prime}$ tungsten skimmer, and a $1,500 \mathrm{l} / \mathrm{s}$ diffusion pump in the differential region containing a $.065^{\prime \prime}$ stainless steel skimmer. The main chamber is pumped by two liquid nitrogen baffled $3,000 \mathrm{l} / \mathrm{s}$ diffusion pumps. A three-axis rotatable manipulator equipped with a surface heater (Vacuum Generators) is mounted in the main chamber so that the axis of rotation lies perpendicular to and at the intersection of the molecular beam and the axis of the detector. A cross-correlation mechanical chopper wheel lies in between the surface and the detector to chop the atoms recoiling from the surface, allowing for time-offlight measurements. ${ }^{13}$ The flight length from the chopper wheel to the ionizer is $23.9 \mathrm{~cm} .{ }^{14}$ The UHV detector is a triply differentially pumped quadrupole mass 
spectrometer (Extrel), with each region pumped by a $400 \mathrm{l} / \mathrm{s}$ magnetically suspended turbomolecular pump (Seiko-Seiki) collectively backed with a Diffstak (Edwards Model 63). A Brink type electron bombardment ionizer with an effective length of $1.5 \mathrm{~cm}$, housed in a liquid nitrogen cooled copper insert, ionizes atoms (ionization efficiency is $10^{-4}$ ) as they fly in from the interaction region. Electrostatic lenses guide newly formed ions entering and exiting the quadrupole filter, and a channeltron electron multiplier (Galileo) multiplies the ion signal. The pulses from the channeltron are sent to a multichannel scaler (Stanford Research SR430), which is interfaced through a GPIB interface (National Instruments) to a 486-25 PC, where time-of-flight data can be co-added and uncorrelated from the pseudo-random sequence of the cross-correlation chopper wheel. ${ }^{13}$

\section{Results}

For each surface, the time-of-flight spectra of recoiling $\mathrm{He}$ atoms and $\mathrm{Ne}$ atoms were taken as a function of incident energy, while holding the angles $\theta_{\mathrm{i}}$ and $\phi_{\mathrm{i}}$ fixed at $45^{\circ}$ and $0^{\circ}$, respectively. The energy transfer as a function of collision energy was investigated in this measurement. The time-of-flight spectra were also taken at fixed collision energy and $\theta_{\mathrm{i}}$ for varying $\phi_{\mathrm{i}}$. Some features of the time-offlight spectra are more distinguishable at certain values of $\phi_{\mathrm{i}}$. Time-of-flight spectra were also measured at a fixed collision energy and $\phi_{\mathrm{i}}$ over a range of $\theta_{\mathrm{i}}$, to determine whether energy is preferentially lost in the parallel or perpendicular directions to the surface. In all the following experiments, the integrated signal 
from the time-of-flight spectra as a function of $\theta_{i}$ has a maximum at $\theta_{i}=45^{\circ}$, indicating that energy is lost equally in the parallel and perpendicular directions to the surface. The results for the $\theta_{\mathrm{i}}$ dependence of the time-of-flight spectra are independent of collision energy. Since the systems have the same $\theta_{\mathrm{i}}$ dependence, only one example, the case of $\mathrm{Ne}$ atoms scattered from $\mathrm{NaCl}$, is illustrated below.

\section{Scattering from LiF(001)}

$\mathrm{He}$ atom scattering from $\mathrm{LiF}(001)$ was measured in the range from 3-20 $\mathrm{kcal} / \mathrm{mole}$ for $\theta_{\mathrm{i}}=45^{\circ}$ and $\phi_{\mathrm{i}}=0^{\circ}$. The crystal temperature was maintained at $656-$ $666 \mathrm{~K}$ to prevent adsorption of water or other background gases. Two time-offlight specta are shown in Fig. 3 , one for $E_{\text {coll }}=3 \mathrm{kcal} / \mathrm{mole}$ (Fig. $3 \mathrm{a}$ ) and one for $E_{\text {coll }}=20 \mathrm{kcal} / \mathrm{mole}$ (Fig. $3 \mathrm{~b}$ ). The time-of-flight spectra for $\mathrm{He}$ atoms scattered from $\mathrm{LiF}(001)$ show only one peak. When the time-of-flight spectra from Fig. 3 are converted into translational energy distributions, Fig. 4, the peak energy and the maximum energy of the scattered beam can be determined by the leading edge in the time-of-flight spectrum. The translational energy distribution is calculated using the method of forward convolution, taking into account instrumental parameters such as the spread in the time-of-flight due to the width of the ionizer. ${ }^{15}$

In the low collision energy He atom time-of-flight spectra, it is clear that the maximum energy extends out beyond the incident beam energy, Fig.3a. In Figs.4a and $4 \mathrm{~b}$, two translational energy distributions are shown, corresponding to the two channels used in constructing the fit to the time-of-flight spectrum. The second 
channel (Fig.4b) is a Maxwell-Boltzmann distribution for the surface temperature of the crystal, and the first channel (Fig.4a) is adjusted so that the total fit matches the data. Notice that for the second channel, the Maxwell-Boltzmann distribution, the translational energy distribution extends out to energies corresponding to the leading edge of the time-of-flight spectrum. However, to get a good total fit, the first channel must also extend out to energies beyond the collision energy. There are therefor atoms that gain energy from the hot surface. This effect is reproduced in molecular dynamics simulations that were performed to try and better understand the experimental results. ${ }^{16}$ The percentage of the energy transferred, $\% \mathrm{E}_{\text {trans. }}$, based on the collision energy and the peak maximum in the translational energy distribution for recoiling atoms is calculated according to :

$$
E_{\text {trans. }}=\frac{E_{\text {coll }}-E_{P(E)}}{E_{\text {coll }}},
$$

and the results are in displayed as the open circles in Fig. 5.

The variation of the time-of-flight spectrum. with $\phi_{\mathrm{i}}$ was investigated at $\mathrm{E}_{\text {coll }}=$ $12 \mathrm{kcal} /$ mole for $\theta_{\mathrm{i}}=20^{\circ}, 45^{\circ}$, and $60^{\circ}$. There is not much of a shift in the leading edge of the time-of-flight with changing $\phi_{i}$, however the intensity of the scattering peak decreases as $\phi_{i}$ is increased, Fig. 6 . The change in the time-of-flight peak maximum with $\phi_{i}$ is quite small, especially for $\theta_{i}=45^{\circ}$, in both the experiments and the simulations for He atom scattering. Variations of the spectra with $\phi_{i}$ are generally more clearly seen at small and large values of $\theta_{\mathrm{i}}$, i.e. away from $\theta_{\mathrm{i}}=45^{\circ}$. 
$\mathrm{Ne}$ atom scattering from $\operatorname{LiF}(001)$ was investigated in the range of beam energies $13-66 \mathrm{kcal} / \mathrm{mole}$. Time-of-flight spectra for the $\mathrm{Ne}$ atoms have a fast sharp peak and a slow tail. When these time-of-flight spectra are transformed into translational energy distributions, it is clear that there are two energy loss peaks, one that involves a relatively small energy loss (fast time-of-flight peak) and one that is almost $100 \%$ energy loss (slow time-of-flight peak). In Fig. 7a, a $\mathrm{Ne}$ atom time-of-flight is shown with a corresponding translational energy distribution for $\theta_{i}=20^{\circ}$ (Fig. $7 \mathrm{~b}$ ). At $\theta_{i}=20^{\circ}$ slow Ne atoms are clearly seen in the time-of-flight spectrum. The slow peak is not as clear in the time-of-flight spectrum when $\theta_{\mathrm{i}}$ is closer to $45^{\circ}$, but is still seen when transforming the time-of-flight data into a translational energy distribution. The slow part of the time-of-flight spectrum cannot simply be fit to a Maxwell-Boltzmann distribution for the surface temperature in this case. The energy loss of $\mathrm{Ne}$ atoms was measured as a function of collision energy for fixed $\theta_{i}=45^{\circ}$ and $\phi_{i}=0^{\circ}$ with crystal temperatures in the range of $659-664 \mathrm{~K}$. The results are presented as the open squares and diamonds in Fig. 5.

Time-of-flight data for $E_{\text {coll }}=45 \mathrm{kcal} / \mathrm{mole}$ were taken at $\theta_{i}=20^{\circ}, 45^{\circ}$, and $60^{\circ}$ for $\phi_{\mathrm{i}}$ values between $0^{\circ}$ and $40^{\circ}$. The experimental findings show that at $\theta_{\mathrm{i}}=$ $20^{\circ}$ and $45^{\circ}$ there is no noticeable $\phi_{i}$ dependence, but for $\theta_{i}=60^{\circ}$, the peak position and leading edge of the time-of-flight change with $\phi_{i}$, Fig 8 . There is a small peak shift to longer times as $\phi_{\mathrm{i}}$ increases from $0^{\circ}$ to $40^{\circ}$. In addition, the intensity for 
the $\phi_{i}=0^{\circ}$ peak is larger than the intensities for the larger values of $\phi_{i}$, which are similar to one another.

\section{Scattering from $\mathrm{NaCl}(001)$}

The collisional energy dependence of $\mathrm{He}$ atom scattering from $\mathrm{NaCl}(001)$ was investigated for beam energies in the range $3-20 \mathrm{kcal} / \mathrm{mole}$. As in the case of scattering from $\mathrm{LiF}(001)$, only one time-of-flight peak is seen, corresponding to one

peak in the translational energy distribution. Fig. 5 (filled circles) shows the results of the collisional energy dependence study for $\theta_{i}=45^{\circ}$ and $\phi_{i}=0^{\circ}$ with crystal temperatures from 640-654 K. Figs. $9 a$ and $9 b$ show example time-of-flight specta at 3 and $20 \mathrm{kcal} / \mathrm{mole}$ collision energies, respectively, and corresponding translational energy distributions are shown in Figs. 10a, 10b, and 10c. Again, the behavior of the He atoms scattered at low collision energies indicates that some energy is gained from the surface, just as in the case of He atoms scattered from $\operatorname{LiF}(001)$

The scattering of $\mathrm{Ne}$ atoms from $\mathrm{NaCl}(001)$ in the range of 13-66 $\mathrm{kcal} / \mathrm{mole}$ results in time-of-flight spectra with two contributions as seen for $\mathrm{Ne}$ atoms scattered from $\operatorname{LiF}(001)$, Fig. 11. The energy loss as $E_{\text {coll }}$ is varied, determined by translational energy distributions, is summarized in Fig. 5 (filled squares and diamonds) for fixed $\theta_{i}=45^{\circ}$ and $\phi_{i}=0^{\circ}$ with crystal temperatures of $640-651 \mathrm{~K}$.

The $\phi_{\mathrm{i}}$ dependence of $\mathrm{Ne}$ atom scattering from $\mathrm{NaCl}(001)$ was investigated for $\theta_{i}=20^{\circ}, 45^{\circ}$, and $60^{\circ}$ at a collision energy of $E_{\text {coll }}=45 \mathrm{kcal} / \mathrm{mole}$. The $\phi_{i}$ 
dependence is shown in Fig. 12 for $\theta_{i}=60^{\circ}$, where the time-of-flight peak is shifted (both the peak maximum and leading edge) to shorter times for $\phi_{i}=0^{\circ}$ as compared to $\phi_{i}=40^{\circ}$. Also, the peak intensity is greatest for $\phi_{i}=0^{\circ}$ and least for $\phi_{i}=40^{\circ}$.

Time-of-flight spectra taken for $\mathrm{Ne}$ atoms scattered from $\mathrm{NaCl}(001)$ for a range of $\theta_{i}$ and a collision energy of $13 \mathrm{kcal} / \mathrm{mole}$ was measured by integrating the area under each time-of-flight spectrum for every value of $\theta_{\mathrm{i}}$ while holding $\phi_{i}=0^{\circ}$ and the crystal temperature at $654-656 \mathrm{~K}$. As can be seen from Fig. 13, the maximum intensity is at $45^{\circ}$. This behavior was seen for the other systems as well. Keep in mind that the source-to-detector angle is fixed at $90^{\circ}$, so that the angular distribution is not the total flux as would be the case if the detector measured scattering over the entire solid angle that originates from the interaction region.

\section{Discussion}

In the classical impulsive collision model, energy transfer is dependent on the masses of the collision partners involved and not on the collision energy. The energy transfer to a surface based on Eqn. 4 results from a simple billiard ball type picture. In this simple picture the energy transfer between the incoming atom and an ion sitting on the surface of the crystal can be calculated. The predicted energy transfer from this calculation for different atom-ion collisions is shown on the right side of Fig. 5 (93\% for $\mathrm{He}-\mathrm{Li} ; 57 \%$ for $\mathrm{He}-\mathrm{F} ; 50 \%$ for $\mathrm{He}-\mathrm{Na} ; 36 \%$ for $\mathrm{He}-\mathrm{Cl}$; $\sim 100 \%$ for $\mathrm{Ne}-\mathrm{F} ; 99 \%$ for $\mathrm{Ne}-\mathrm{Na}$; and $93 \%$ for $\mathrm{Ne}-\mathrm{Cl} ; \mathrm{M}_{\mathrm{a}}>\mathrm{M}_{\mathrm{b}}$ for $\mathrm{Ne}-\mathrm{Li}$ ). However, if the geometrical constraints on the incoming atom as it approaches the 
salt ions are considered, it is clear that the incoming $\mathrm{He}$ atom or $\mathrm{Ne}$ atom will not easily access an exposed cation in the salt in the case of a perfectly flat surface, Fig. 14. In both $\mathrm{LiF}(001)$ and $\mathrm{NaCl}(001)$, the anion eclipses the cation. The ionic radii in $\mathrm{LiF}$ are $\mathrm{r}_{\mathrm{Lit}}=0.60 \AA$ and $\mathrm{r}_{\mathrm{F}-}=1.36 \AA$; in $\mathrm{NaCl}$ they are $\mathrm{r}_{\mathrm{Na}+}=0.95 \AA$ and $\mathrm{r}_{\mathrm{Cl}}=$ $1.81 \AA .{ }^{17}$ The incoming particles, He atoms and Ne atoms, have atomic radii of $\sim 1.5$ and $1.58 \AA$, respectively. Thus, an incoming $\mathrm{He}$ atom or Ne atom will not see an exposed $\mathrm{Li}^{+}$or $\mathrm{Na}^{+}$unless the cation is sitting at an exposed terrace site, where it is not eclipsed by any anion neighbors. This explains why two distinct peaks are not seen for He atoms scattered from $\operatorname{LiF}(001)$ with one peak corresponding to impulsive He-Li collisions, for example.

He atom scattering from $\mathrm{LiF}(001)$ and $\mathrm{NaCl}(001)$ shows similar patterns of energy transfer, as seen in Fig. 5. The energy transfer for He atom scattered from $\mathrm{LiF}(001)$ falls short of the $57 \%$ predicted for an impulsive He-F collision, which could be due to two factors. ${ }^{18}$ First of all, the surface is hot, and thus the energy transfer is expected to be reduced by an amount on the order of $k_{B} T_{s}$, where $T_{s}$ is the surface temperature. Second, the predictions from Eqn. (4) result from a one dimensional model, and extending to three dimensions and averaging over impact parameter will reduce $\Delta \mathrm{E} / \mathrm{E}$. The energy transfer for He atoms scattered from $\mathrm{NaCl}(001)$ goes above the $36 \%$ predicted for a $\mathrm{He}-\mathrm{Cl}$ collision. If Eqn. (4) is used to try to correlate the observed $\Delta \mathrm{E} / \mathrm{E}$ with multiple collisions, the observed $\Delta \mathrm{E} / \mathrm{E}$ would correspond to up to two sequential collisions. However, the amount of energy transfer steadily rises with collision energy in the range of $3-20 \mathrm{kcal} / \mathrm{mole}$, 
which is inconsistent with the impulsive model.

$\mathrm{Ne}$ atom scattering from $\mathrm{LiF}(001)$ and $\mathrm{NaCl}(001)$ was different from $\mathrm{He}$ atom scattering in that two types of energy loss were clearly observed instead of just one. In both cases, the fast time-of-flight peak corresponded to energy loss that is less efficient than what the impulsive model predicts for a simple gas atomanion head-on collision. This first energy loss channel only increased moderately as the collision energy was increased from $13-66 \mathrm{kcal} / \mathrm{mole}$, levelling off at higher collision energies to $65-70 \%$ energy transfer. The slow time-of-flight feature corresponds to more efficient energy loss, and more closely resembles what the impulsive model predicts. This energy loss channel does not change appreciably with collision energy. The atoms that undergo the second type of collision lose almost all memory of their incident velocity. They are sligtly faster than atoms that simply desorb from a surface at the surface temperatures used, but the broad distribution reflects the fact that the energy left in translation has a broad MaxwellBoltzmann type distribution. The broad peaks in the time-of-flight also result from the fact that some atoms may undergo multiple collisions, and therefor a range of energy transfer is observed. Thus the time-of-flight is quite broad for the slow component. The slow recoiling $\mathrm{Ne}$ atoms are expected especially for $\mathrm{Ne}-\mathrm{F}$ collisions, where the masses of the $\mathrm{Ne}$ atom and $\mathrm{F}$ atom are nearly equal; collisions between atoms of similar mass are the most efficient at transferring energy (in Eqn.4, as $M_{a}->M_{b}, \Delta E / E->1$ ). However, a highly efficient energy transfer single collision cannot be distinguished from high energy loss due to 
multiple scattering based on the time-of-flight data. This is one of the motivations for doing molecular dynamics simulations.

The faster time-of-flight peak for the Ne atom scattering off each salt results from a less efficient energy transfer collision. Since in the case if $\mathrm{Ne}$ atoms, $\mathrm{k}_{\mathrm{B}} \mathrm{T}_{\mathrm{s}}$ $<<E_{\text {coll }}$, the elevated surface temperature is not expected to lead to a significant decrease in the energy transfer. However, the effect of averaging over impact parameter, as already mentioned, still applies here. The reduced energy transfer would also occur if several atoms worked in concert, as a coupled set, during the collision, for then the incoming atom sees a greater effective mass as its collision partner. For example, in the $\mathrm{Ne}$ atom scattering from $\mathrm{LiF}(001)$ at $66 \mathrm{kcal} / \mathrm{mole}$ collision energy, the first channel resulted in $70 \%$ energy transfer to the surface (Fig. 5). So, if $\Delta \mathrm{E} / \mathrm{E}$ is set to 0.70 in Eqn. 4 , the effective mass $\left(\mathrm{M}_{\text {eff }}\right)$ at the surface that the Ne atom collided with $\left(\mathrm{M}_{\text {eff }}=\mathrm{M}_{\mathrm{b}}\right)$ can be calculated to be $\mathrm{M}_{\text {eff }} \sim 68$. For $64 \%$ energy transfer, when $\mathrm{E}_{\text {coll }}=45 \mathrm{kcal} / \mathrm{mole}, \mathrm{M}_{\text {eff }}=80$. Although $\mathrm{M}_{\text {eff }}$ does not tell us which atoms are acting together, it is useful to see that as the $\Delta E / E$ gets larger, $\mathrm{M}_{\text {eff }}$ decreases. The width of the fast time-of-flight peak comes from scattering over a range of impact parameters governed by the local surface morphology, which includes steps and defects visible on the atomic level. ${ }^{19}$

The dependence of the time-of-flight spectra on the two angles, $\theta_{\mathrm{i}}$ and $\phi_{\mathrm{i}}$, was explored for all the systems. For all four systems, He-LiF(001), Ne-LiF(001), He- $\mathrm{NaCl}(001)$, and $\mathrm{Ne}-\mathrm{NaCl}(001)$, the largest integrated time-of-flight signal occurred at $\theta_{\mathrm{i}}=45^{\circ}$ when the collision energy, $\phi_{\mathrm{i}}$, and the crystal temperature were 
held constant. The shape of the $\theta_{i}$ dependence does not change in the range of collision energies studied here. For the fixed geometry in this experiment, and given that the $\theta_{\mathrm{i}}$ behavior is independent of $\mathrm{E}_{\mathrm{coll}}$ (i.e. the scattering lobe does not change with $E_{\text {coll }}$ ) this type of angular distribution indicates whether energy is lost preferentially in the parallel or perpendicualar directions to the surface. In Fig. 15, three types of $\theta_{i}$ dependences are shown: Case 1) energy is lost equally in both the parallel and perpendicular directions; Case 2) energy is preferentially lost along the direction perpendicular to the surface; Case 3) energy is preferentially lost in the direction parallel to the surface. As $\theta_{i}$ is changed from $45^{\circ}$, as shown in the figure, the detectable product vector also changes so that the entire lobe may be scanned within a given plane. Thus, the maximum in the lobe is found in the beam-detector plane. The assumptions here are that the scattering lobe is cylindrically symmetric, which is a reasonable assumption since scattering of this type usually gives a $\operatorname{Cos}^{\mathrm{n}} \theta$ type distribution, and that the lobe does not change with incident angle.

The $\phi_{\mathrm{i}}$ dependence was explored when it became apparent that the time-offlight shifted with $\phi_{\mathrm{i}}$ in the molecular dynamics simulations. The shift with $\phi_{\mathrm{i}}$ is most easily seen for $\mathrm{Ne}$ atom scattering, especially in the case of $\mathrm{Ne}$ atoms scattered from $\mathrm{NaCl}(001)$, Fig. 12. Here, energy transfer is slightly less efficient when scattering is along the $(001)$ direction of the lattice. This could perhaps be due to increased coupling to the nearest neighboring atoms along the (001) direction, creating a slightly higher $M_{\text {eff. }}$ In all the cases shown in Figs. 6, 8, and 12 , the scattering signal is greatest when $\phi_{i}=0^{\circ}$. Since the detector-to-source angle 
is fixed in one plane at $90^{\circ}$, this indicates that more of the scattered atoms stay in the scattering plane along the (001) direction than along the other directions defined by $\phi_{\mathrm{i}}$.

\section{Conclusion}

The atomic beam surface scattering experiments described in this chapter show the different mechanisms of energy transfer for hyperthermal He atoms and Ne atoms colliding with $\mathrm{LiF}(001)$ and $\mathrm{NaCl}(001)$. He atom scattering for both surfaces shows only one distinguishable time-of-flight peak and similar energy transfer behavior as a function of collision energy. ${ }^{20}$ For He atoms scattered from $\mathrm{LiF}(001)$, the $\Delta \mathrm{E} / \mathrm{E}$ approaches but falls short of the $57 \%$ predicted for an impulsive $\mathrm{He}-\mathrm{F}$ collision. For $\mathrm{He}$ scattered from $\mathrm{NaCl}(001)$, the energy transfer goes above the $36 \%$ predicted for an impulsive $\mathrm{He}-\mathrm{Cl}$ collision. In both cases $\Delta \mathrm{E} / \mathrm{E}$ rises with $\mathrm{E}_{\text {coll }}$. Ne atom scattering differed from He atom scattering in that two contributions to the time-of-flight could be distinguished. Each Ne atom timeof-flight had a fast sharp peak and a slow broad peak. Once $\mathrm{E}_{\text {coll }}$ was about 20 $\mathrm{kcal} / \mathrm{mole}$, energy transfer did not change appreciably with collision energy, which is consistent with the impulsive model. The fast time-of-flight peak results from less efficient energy transfer than predicted by the impulsive model for atom-anion collisions, and can be rationalized as scattering from a coupled set of atoms working together as one collision partner. The slow time-of-flight peak, from atoms that lose almost all their energy to the surface, is consistent with an atom- 
anion impulsive collision but could also be due to multiple collisions.

The dependence of scattering on the angles $\theta_{i}$ and $\phi_{i}$ showed that scattered flux was greatest for $\theta_{i}=45^{\circ}$ and $\phi_{i}=0^{\circ}$. A slight shift in the time-of-flight with varied $\phi_{\mathrm{i}}$, was also observed. 


\section{References and Notes}

1. F. P. Tully, N. H. Cheung, H. Haberland, and Y. T. Lee, J. Chem. Phys. 73, $4460(1980)$.

2. B. H. Mahan, J. Chem. Phys. 52, 5221 (1970).

3. F. O. Goodman and H. Y. Wachman, Dynamics of Gas-Surface Scattering (Academic Press, NewYork, 1976).

4. A. Amirav, M. J. Cardillo, P. L. Trevor, Carmay Lim, and J. C. Tully, J. Chem. Phys. 87, 1796 (1987).

5. See Chapter 3.

6. Toennies, J. P, Dynamics of Gas-Surface Interaction (Springer Verlag, New York, 1982).

7. L. Mattera, M. Rocca, C. Salvo, S. Terreni, F. Tommasini, and U. Valbusa, Dynamics of Gas-Surface Interaction (Springer Verlag, New York, 1982).

8. M. E. Saecker and G. M. Nathanson, J. Chem. Phys. 100, 3999 (1994); J. Chem. Phys. 99, 7056 (1993).

9. See Chapter 4.

10. R. K. Sparks, Ph. D. Thesis, University of California, Berkeley (1979).

11. R. B. Bernstein, Chemical Dynamics via Molecular Beam and Laser Techniques (Oxford University Press, New York, 1982).

12. Mechanical booster pump donated by Genus Inc. 
13. R. David, K. Kern, P. Zeppenfeld, and G. Comsa, Rev. Sci. Instrum. 57, $2771(1986)$

14. Detector calibration was carried out to tune the electrostatic lenses and measure the flight length by photodissociating $\mathrm{Cl}_{2}$ and $\mathrm{Br}_{2}$ using $355 \mathrm{~nm}$ light from a Nd:YAG laser. Since the Newton circle for the recoiling fragments is so large compared to the velocity vector for the molecular beam, and the bond dissociation energies are well known, photofragment spectra taken at $90^{\circ}$ could be used to measure the flight length by fitting the time-of-flight data to a translational energy distribution in the form of a $\delta$ function for the proper energy release, given by the difference in the photon energy and the bond dissociation energy.

15. A. M. Wodtke, Ph. D. Thesis, University of California, Berkeley, 1986; X. Zhao, Ph. D. Thesis, University of California, Berkeley, 1988; J. D. Myers, Ph. D. Thesis, University of California, Berkeley, 1993.

16. Molecular dynamics simulations were performed by R. S. Sinkovits.

17. L. Pauling, The Nature of the Chemical Bond, 3rd Edition (Cornell University Press, Ithaca, New York, 1980).

18. J. Harris, Dynamics of Gas-Surface Interactions (The Royal Society of Chemistry, London, 1991).

19. Atomic force microscopy has been used to look at the crystal morphology before and after scattering.

20. Note that in the He atom scattering, a small Maxwell-Boltzmann component 
may be hidden under the major time-of-flight peak, but it cannot be seen directly in either the time-of-flight or the corresponding translational energy distribution. 


\section{Figure Captions}

Fig. 2-1 Gas-Surface Scattering Apparatus with channeltron (A), exit lenses (B), liquid nitrogen cooled copper insert (C), quadrupole filter (D), Brink type electron bombardment ionizer followed by entrance lenses $(E)$, hightemperature rhenium nozzle (F), surface mounted on 3-axis rotatable manipulator (not shown) $(\mathrm{G})$, cross-correlation chopper wheel $(\mathrm{H})$, source region pumped by $6000 \mathrm{l} / \mathrm{s}$ diffusion pump (I), differential region pumped by $1000 \mathrm{l} / \mathrm{s}$ diffusion pump (J), main chamber pumped by two $2000 \mathrm{l} / \mathrm{s}$ diffusion pumps equipped with liquid nitrogen baffles $(K, L)$, three regions of differential pumping on the detector pumped by three $400 \mathrm{l} / \mathrm{s}$ magnetically suspended turbomolecular pumps (T1, T2, T3).

Fig. 2-2 Beam characterization using the rhenium nozzle for $0.6 \% \mathrm{Ne} / \mathrm{H}_{2}$ (solid squares) and $1 \% \mathrm{He} / \mathrm{H}_{2}$ (solid circles) as well as nozzle temperature using neat $\mathrm{Ne}$ (open squares). See text for details of $\mathrm{E}_{\text {beam }}$ calculation from time-of-flight data.

Fig. 2-3 (a) Time-of-flight data (circles) for $3 \mathrm{kcal} / \mathrm{mole} \mathrm{He}$ atoms scattered from $\operatorname{LiF}(001)$ taken at $\theta_{\mathrm{i}}=45^{\circ}, \phi_{\mathrm{i}}=0^{\circ}$ and a surface temperature of $656 \mathrm{~K}$. The two channels are derived from the translational energy distributions of Figs. $4 a \& b$. Channel 2 (dashed line) is a Maxwell-Boltzmann distribution for the surface temperature, and channel 1 (dotted line) is adjusted to make 
the total fit (solid line) follow the data.

(b) Time-of-flight data (circles) for $20 \mathrm{kcal} / \mathrm{mole} \mathrm{He}$ atoms scattered from $\mathrm{LiF}(001)$ taken at the same angles and a surface temperature of $661 \mathrm{~K}$. The fit (solid line) corresponds to the translational energy distribution of Fig. 4c.

Fig. 2-4 (a) Translational energy distribution for channel 1 of Fig. 3a.

(b) Translational energy distribution for channel 2 of Fig. 3a. This is a Maxwell-Boltzmann distribution for $656 \mathrm{~K}$, the surface temperature.

(c) Translational energy distribution for Fig. $3 b$.

Fig. 2-5 Energy transfer as a function of collision energy for the systems studied: He atoms scattered from $\operatorname{LiF}(001) \theta_{\mathrm{i}}=45^{\circ}, \phi_{\mathrm{i}}=0^{\circ}$, crystal temperature $=656-666 \mathrm{~K}$ (open circles); He atoms scattered from $\mathrm{NaCl}(001)$ $\theta_{\mathrm{i}}=45^{\circ}, \phi_{\mathrm{i}}=0^{\circ}$, crystal temperature $=640-654 \mathrm{~K}$ (filled circles); Ne atoms scattered from $\operatorname{LiF}(001) \theta_{\mathrm{i}}=45^{\circ}, \phi_{\mathrm{i}}=0^{\circ}$, crystal temperature $=659-664 \mathrm{~K}$ fast TOF peak(open squares) and slow TOF peak (open diamonds); Ne atoms scattered from $\mathrm{NaCl}(001) \theta_{\mathrm{i}}=45^{\circ}, \phi_{\mathrm{i}}=0^{\circ}$, crystal temperature $=640$ $651 \mathrm{~K}$ fast TOF peak(filled squares) and slow TOF peak (filled diamonds); predictions for impulsive collisions between incoming $\mathrm{He}$ atoms and $\mathrm{Ne}$ atoms and individual surface atoms in the $\mathrm{LiF}(001)$ and $\mathrm{NaCl}(001)$ surfaces (filled triangles). 
Fig. 2-6 The $\phi_{\mathrm{i}}$ dependence of He atom scattering from $\operatorname{LiF}(001)$. He atoms scattered at $12 \mathrm{kcal} / \mathrm{mole}$ with $\theta_{\mathrm{i}}=60^{\circ}$, a surface temperature of $659-661 \mathrm{~K}$, and $\phi_{\mathrm{i}}=0^{\circ}$ (inverted triangles), $\phi_{\mathrm{i}}=20^{\circ}$ (diamonds), $\phi_{\mathrm{i}}=30^{\circ}$ (squares), and $\phi_{\mathrm{i}}=$ $40^{\circ}$ (circles).

Fig. 2-7 (a) Time-of-flight data (circles) for Ne atoms scattered from $\operatorname{LiF}(001)$ with $E_{\text {coll }}=45 \mathrm{kcal} / \mathrm{mole}, \theta_{\mathrm{i}}=20^{\circ}, \phi_{\mathrm{i}}=0^{\circ}$, and $\mathrm{T}_{\text {surface }}=666 \mathrm{~K}$. The fit (solid line) is derived from (b).

(b) Corresponding translational energy distribution, showing two distinct peaks.

Fig. 2-8 The $\phi_{\mathrm{i}}$ dependence of Ne atom scattering from $\operatorname{LiF}(001)$. Ne atoms scattered at $45 \mathrm{kcal} / \mathrm{mole}$ with $\theta_{\mathrm{i}}=60^{\circ}$, a surface temperature of $647-652 \mathrm{~K}$, and $\phi_{\mathrm{i}}=0^{\circ}$ (inverted triangles), $\phi_{\mathrm{i}}=20^{\circ}$ (diamonds), $\phi_{\mathrm{i}}=30^{\circ}$ (squares), and $\phi_{\mathrm{i}}=40^{\circ}$ (circles).

Fig. 2-9 (a) Time-of-flight data (circles) for $3 \mathrm{kcal} / \mathrm{mole}$ He atoms scattered from $\mathrm{NaCl}(001)$ for $\theta_{\mathrm{i}}=45^{\circ}, \phi_{\mathrm{i}}=0^{\circ}$, and $\mathrm{T}_{\text {surface }}=641 \mathrm{~K}$. The two channels are derived from the translational energy distributions of Figs. 10a and 10b. Channel 2 (dashed line) is a Maxwell-Boltzmann distribution for the surface temperature, and channel 1 (dotted line) is adjusted to make the total fit (solid line) fit the data. 
(b) Time-of-flight data (circles) for $20 \mathrm{kcal} / \mathrm{mole} \mathrm{He}$ atoms scattered from $\mathrm{NaCl}(001)$ at the same angles and a surface temperature of $654 \mathrm{~K}$. The fit corresponds to the translational energy distribution of Fig. $10 \mathrm{c}$.

Fig. 2-10 (a) Translational energy distribution for channel 1 of part (a).

(b) Translational energy distribution for channel 2 of part (a).

(c) Translational energy distribution for part (b).

Fig. 2-11 (a) Time-of-flight data (circles) for $45 \mathrm{kcal} / \mathrm{mole} \mathrm{Ne}$ atoms scattered from $\mathrm{NaCl}(001)$ at $\theta_{\mathrm{i}}=20^{\circ}, \phi_{\mathrm{i}}=30^{\circ}$, and $\mathrm{T}_{\text {surface }}=616 \mathrm{~K}$. The fit (solid line) is derived from (b).

(b) Translational energy distibution giving rise to the fit of part (a).

Fig. 2-12 The $\phi_{i}$ dependence of $\mathrm{Ne}$ atom scattering from $\mathrm{NaCl}(001)$. $\mathrm{Ne}$ atoms scattered at $45 \mathrm{kcal} / \mathrm{mole}$ with $\theta_{\mathrm{i}}=60^{\circ}$, a surface temperature of $615-$ $626 \mathrm{~K}$, and $\phi_{\mathrm{i}}=0^{\circ}$ (circles), $\phi_{\mathrm{i}}=20^{\circ}$ (triangles), $\phi_{\mathrm{i}}=30^{\circ}$ (diamonds), and $\phi_{\mathrm{i}}=40^{\circ}$ (squares).

Fig. 2-13 Angular distribution for varied $\theta_{\mathrm{i}}$ and fixed $\phi_{\mathrm{i}}=0^{\circ}$ of $13 \mathrm{kcal} / \mathrm{mole}$ $\mathrm{Ne}$ atoms scattered from $\mathrm{NaCl}(001) . \quad \mathrm{T}_{\text {surface }}=654-656 \mathrm{~K}$. 
Fig. 2-14 The $\mathrm{LiF}(001)$ and $\mathrm{NaCl}(001)$ lattices showing the relative sizes of the ions. This shows that the anion eclipses the cation in each lattice, making direct collision with $\mathrm{Li}$ atoms or $\mathrm{Na}$ atoms unlikely, except in the case of defect sites.

Fig. 2-15 Three types of scattering show how the $\theta_{i}$ dependence probes the scattering lobe in the source-detector plane. Case 1: energy is lost equally in the parallel and perpendicular directions to the surface; Case 2: energy is lost preferentilally in the perpendicular direction; Case 3: energy is lost preferentially in the parallel direction. The results in this paper all follow Case 1. 


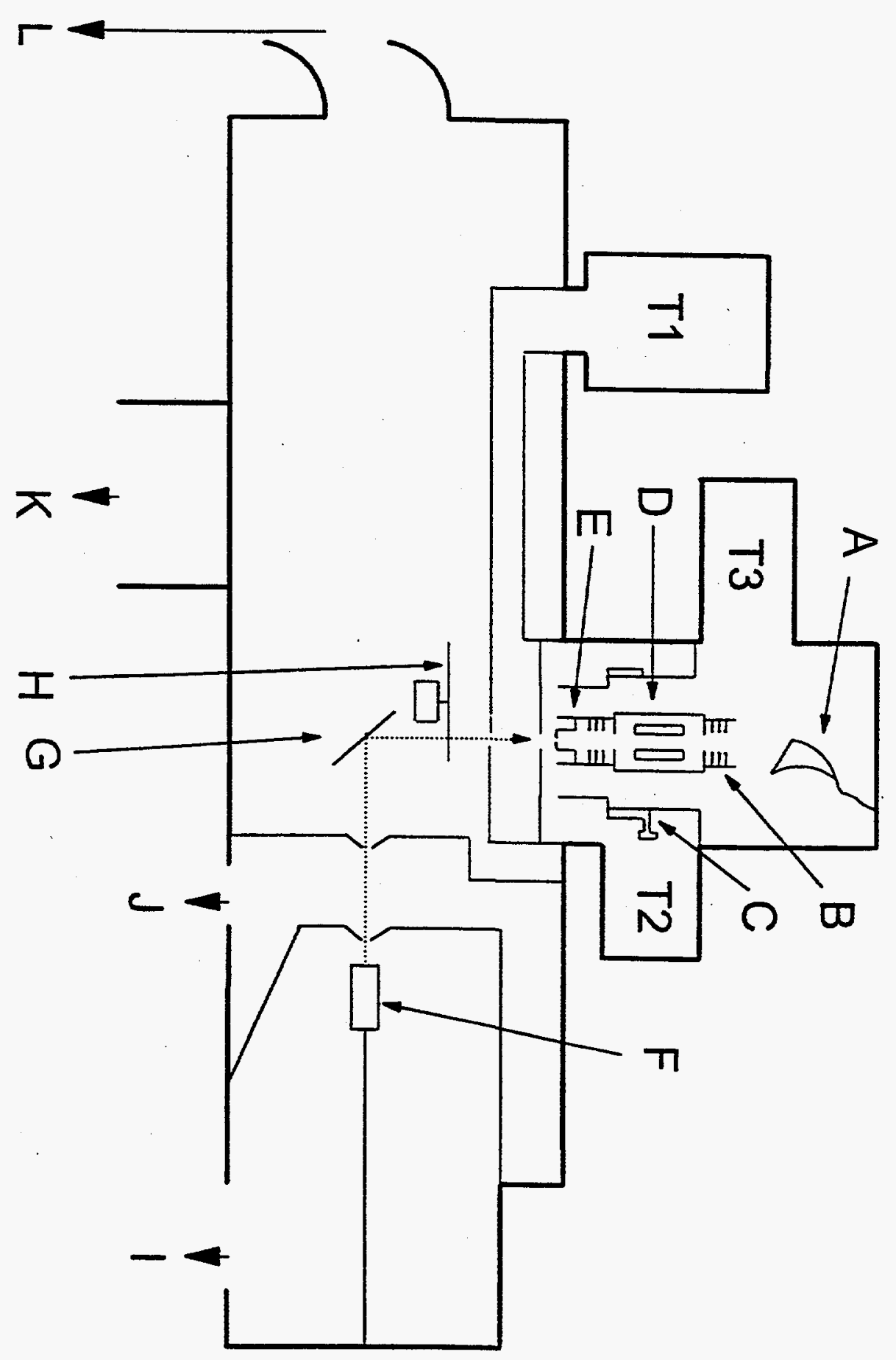

Figure 2-1 


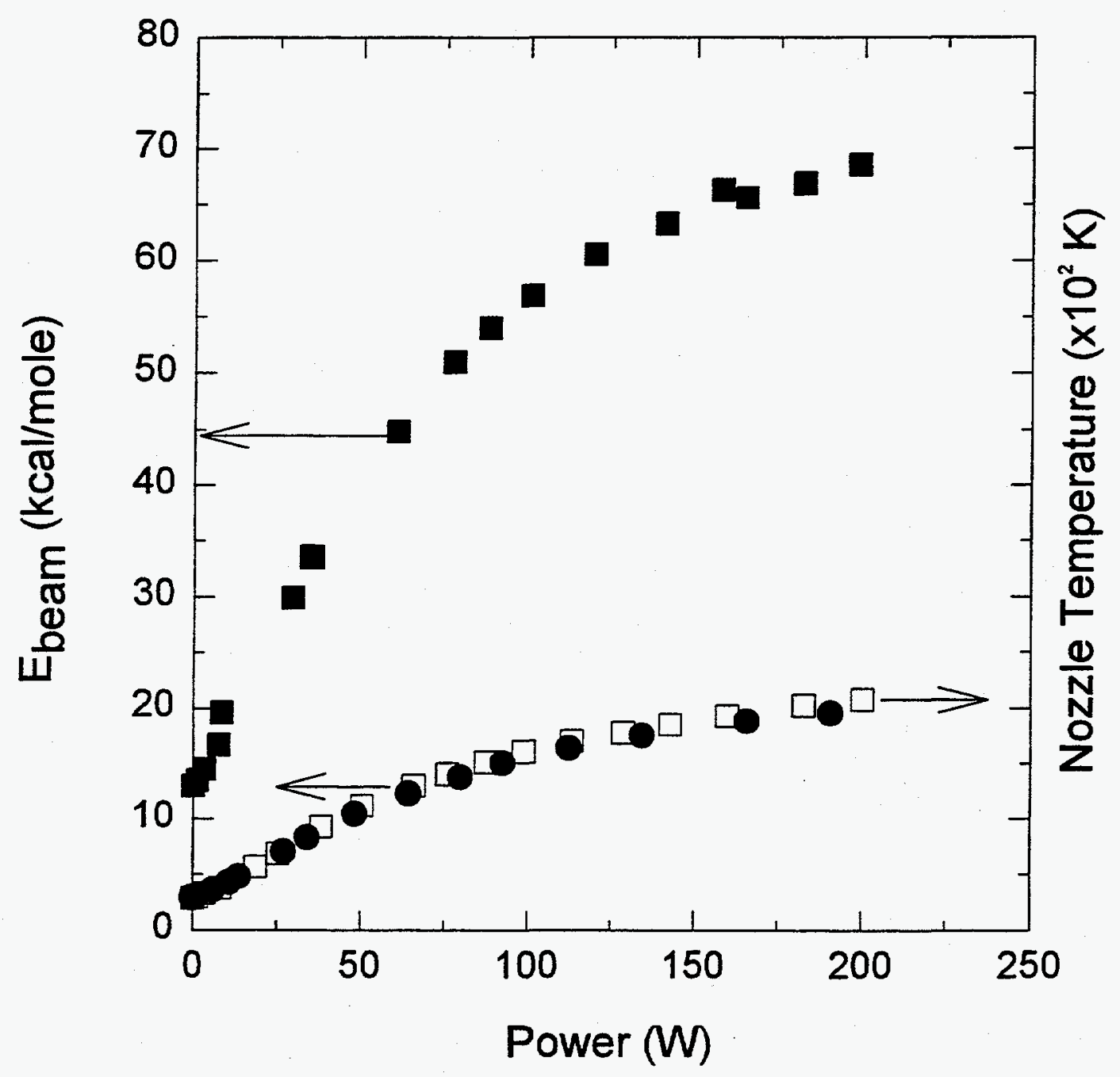

Figure 2-2 


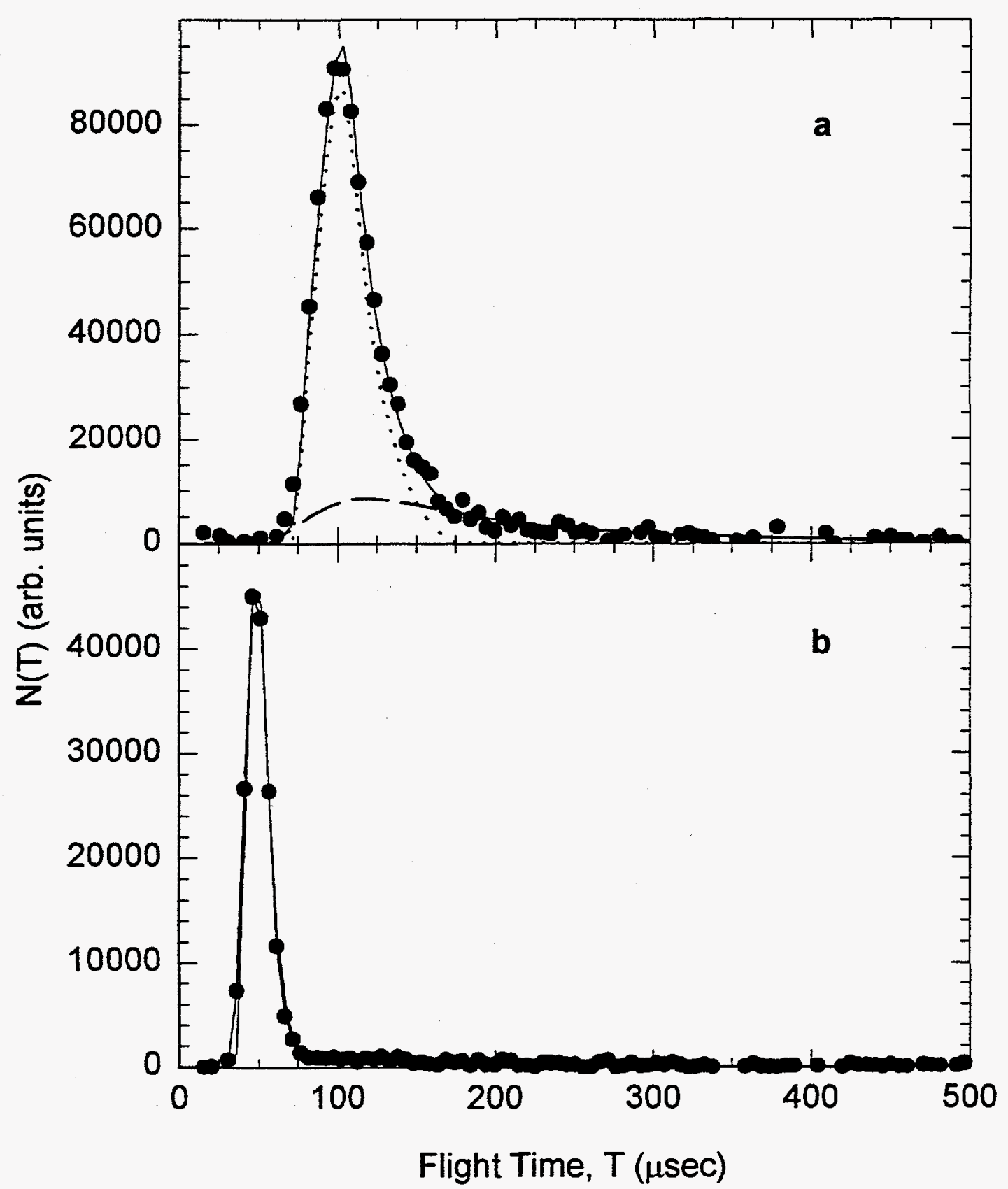

Figure 2-3 


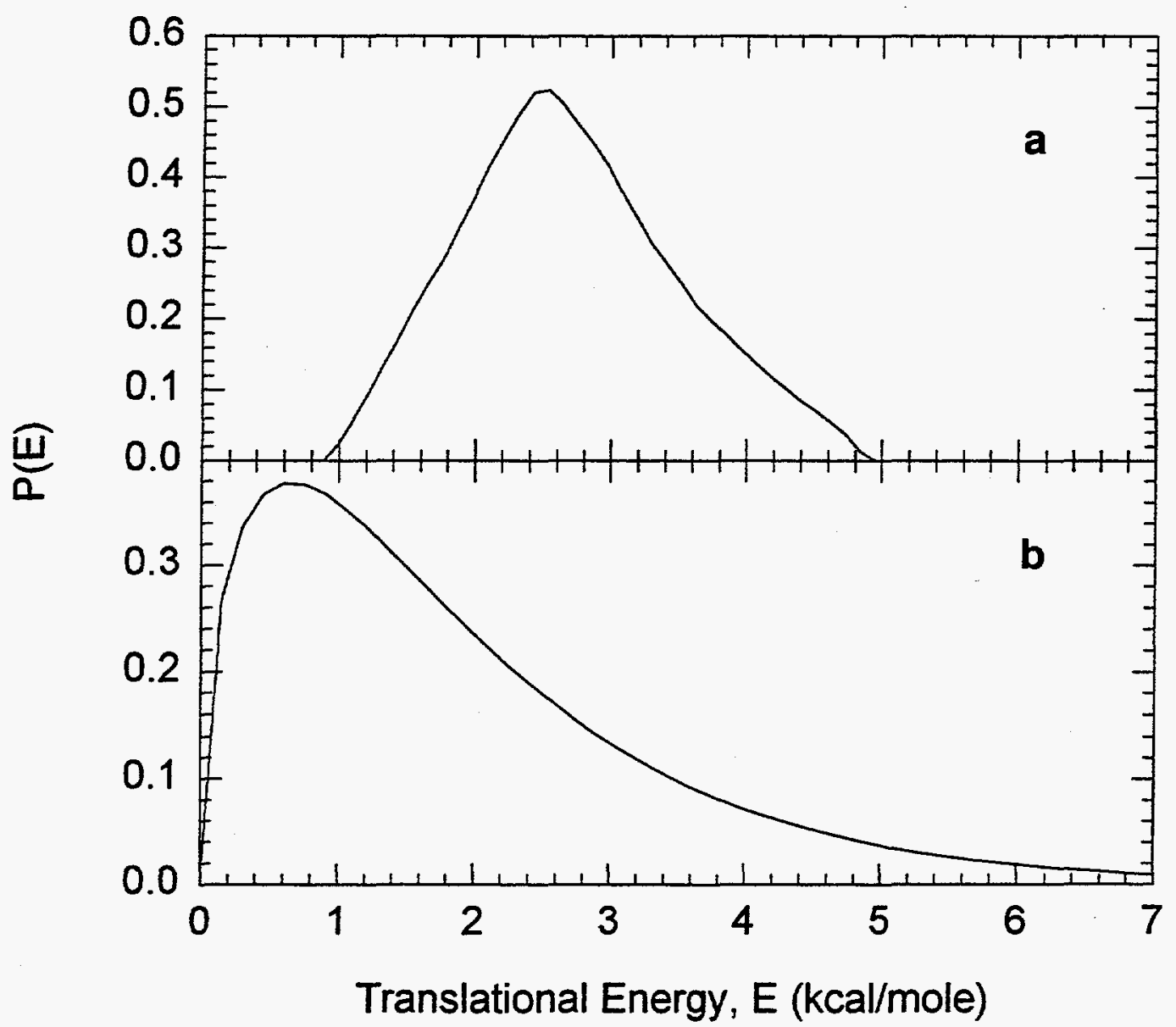

37

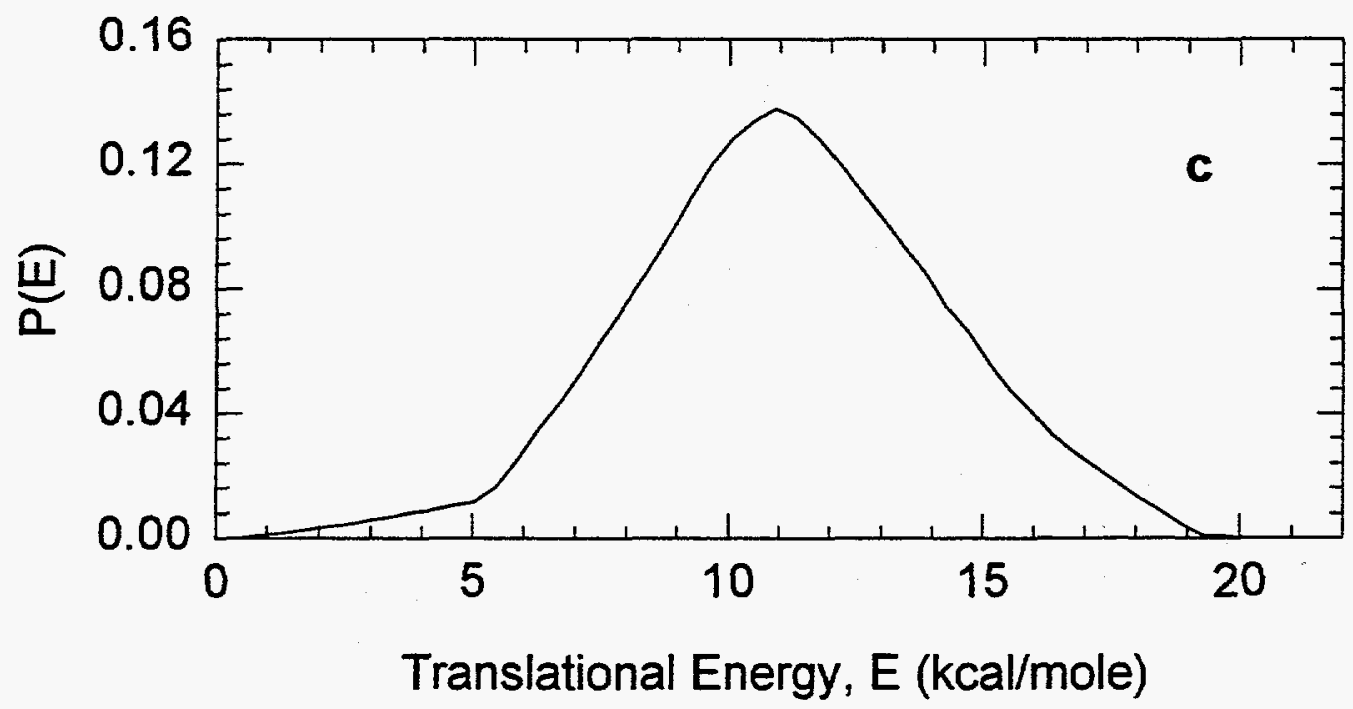

Figure 2-4 


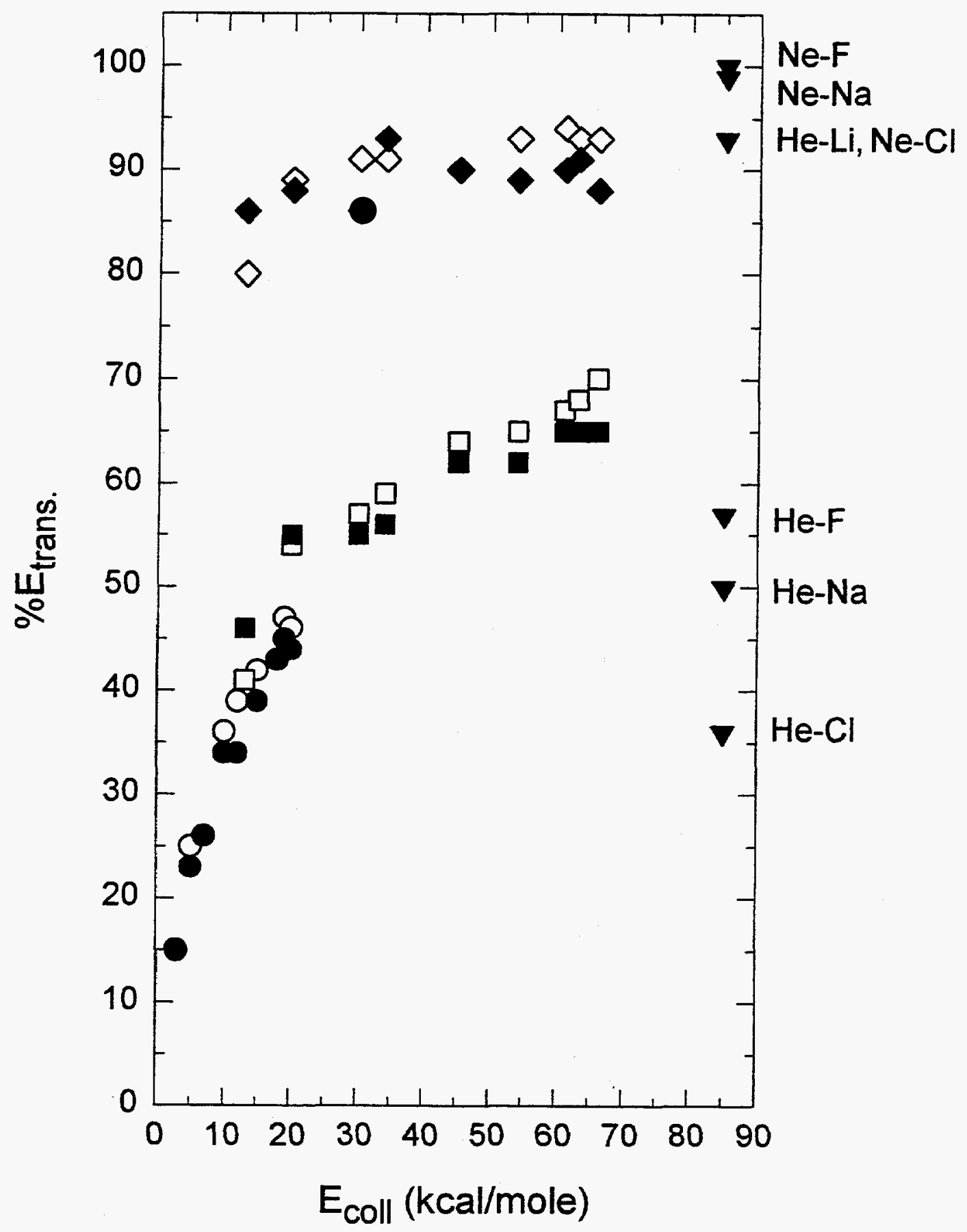

Figure 2-5 


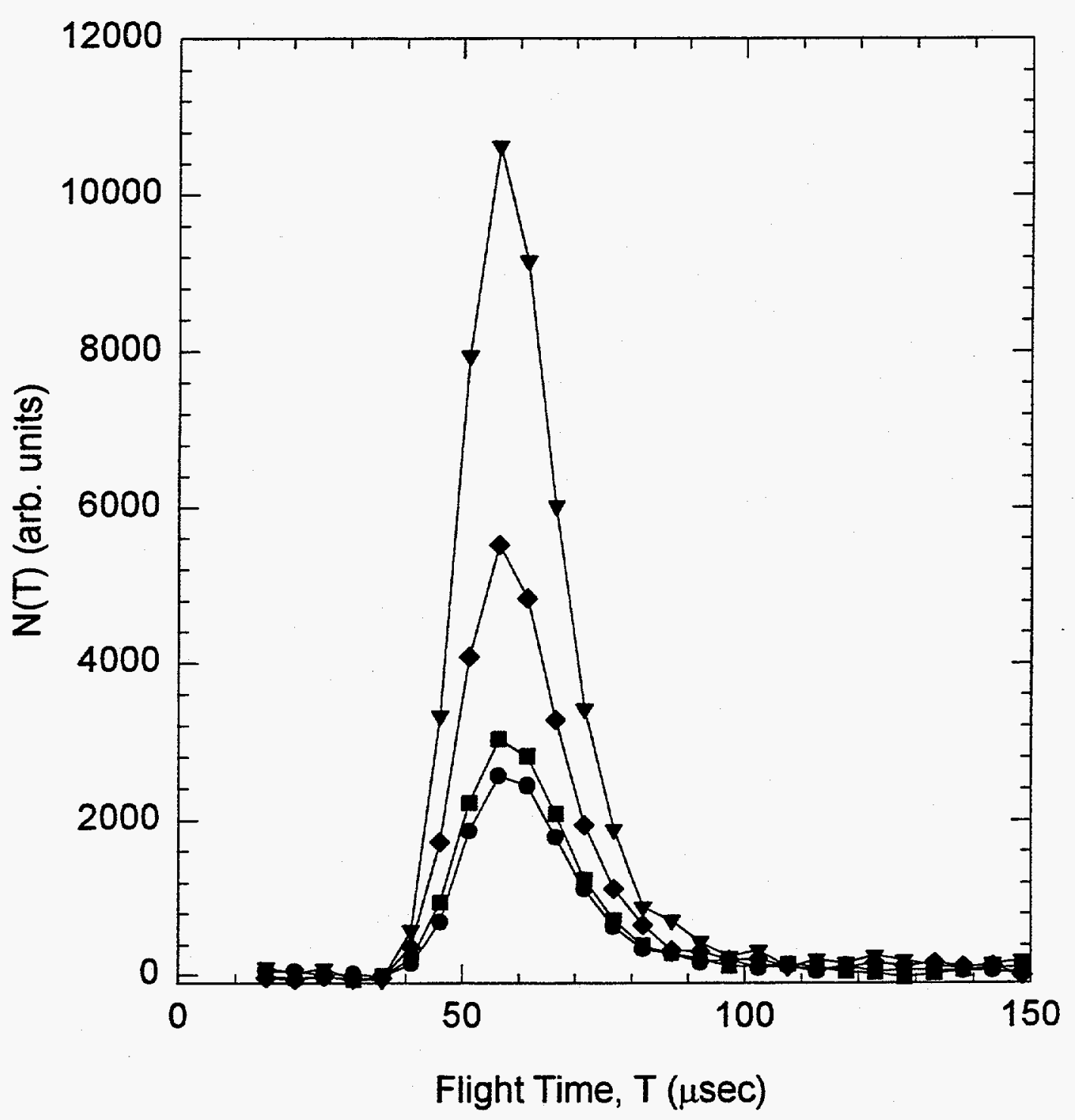

Figure 2-6 


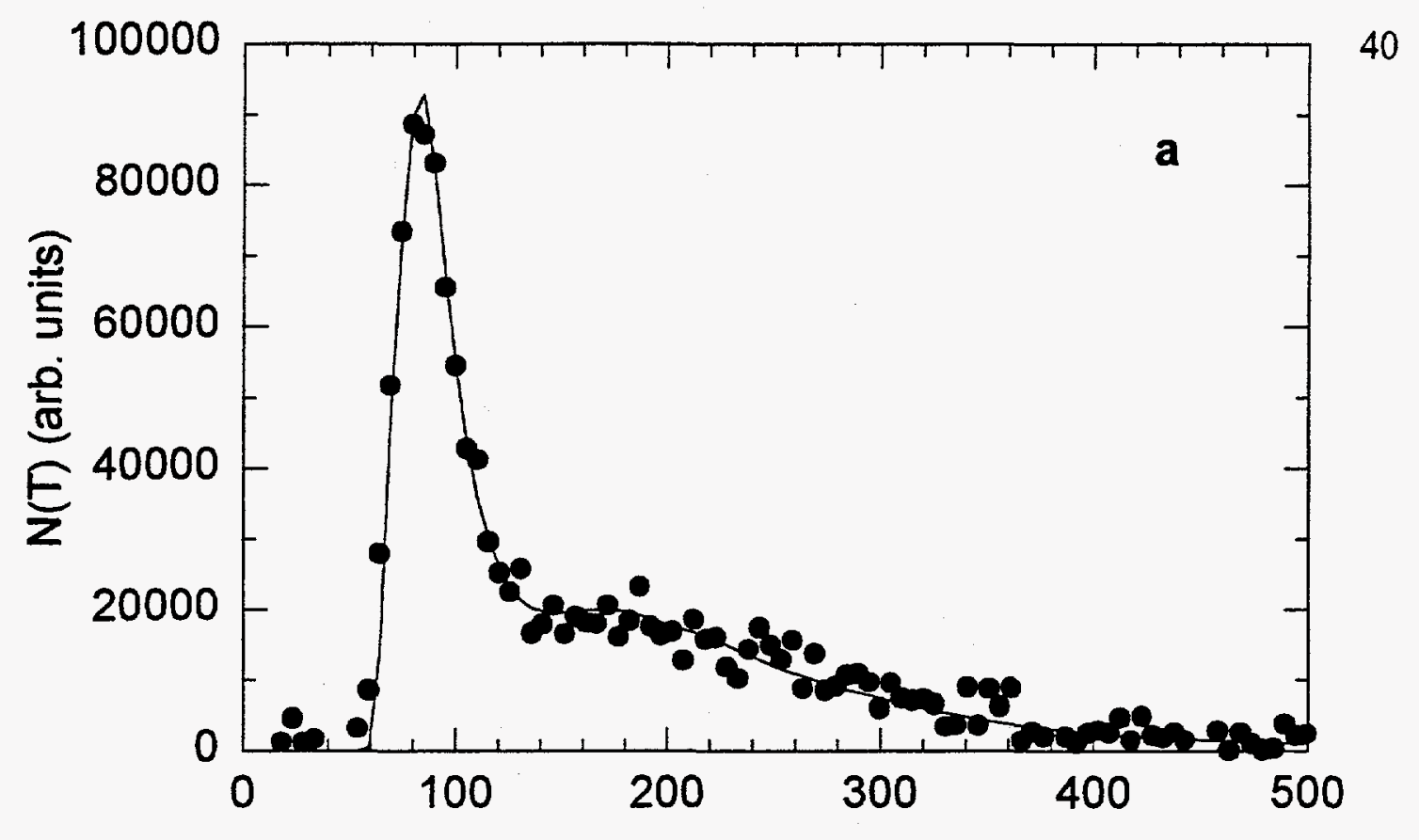

Flight Time, $\mathrm{T}$ ( $\mu \mathrm{sec})$

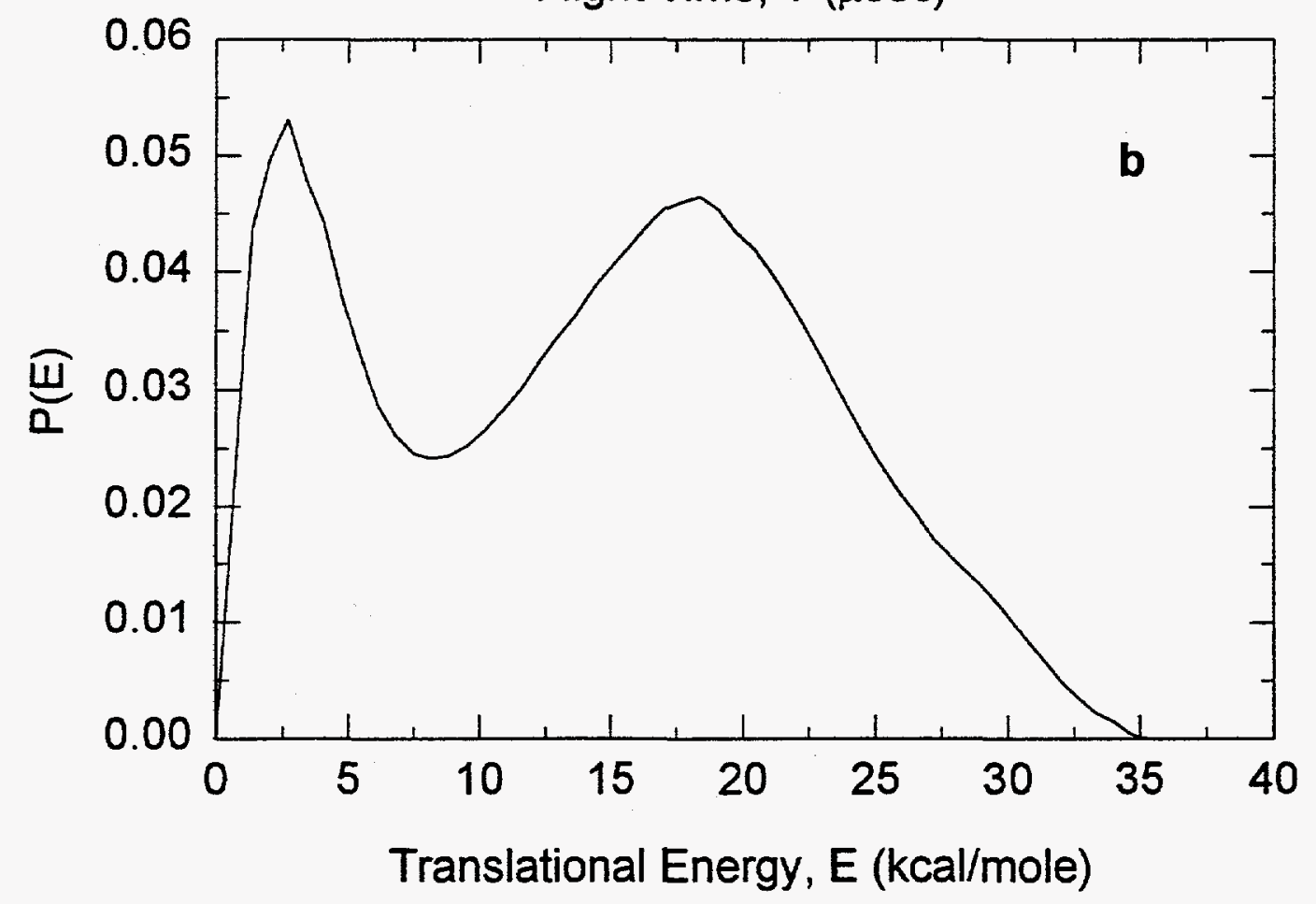

Figure 2-7 


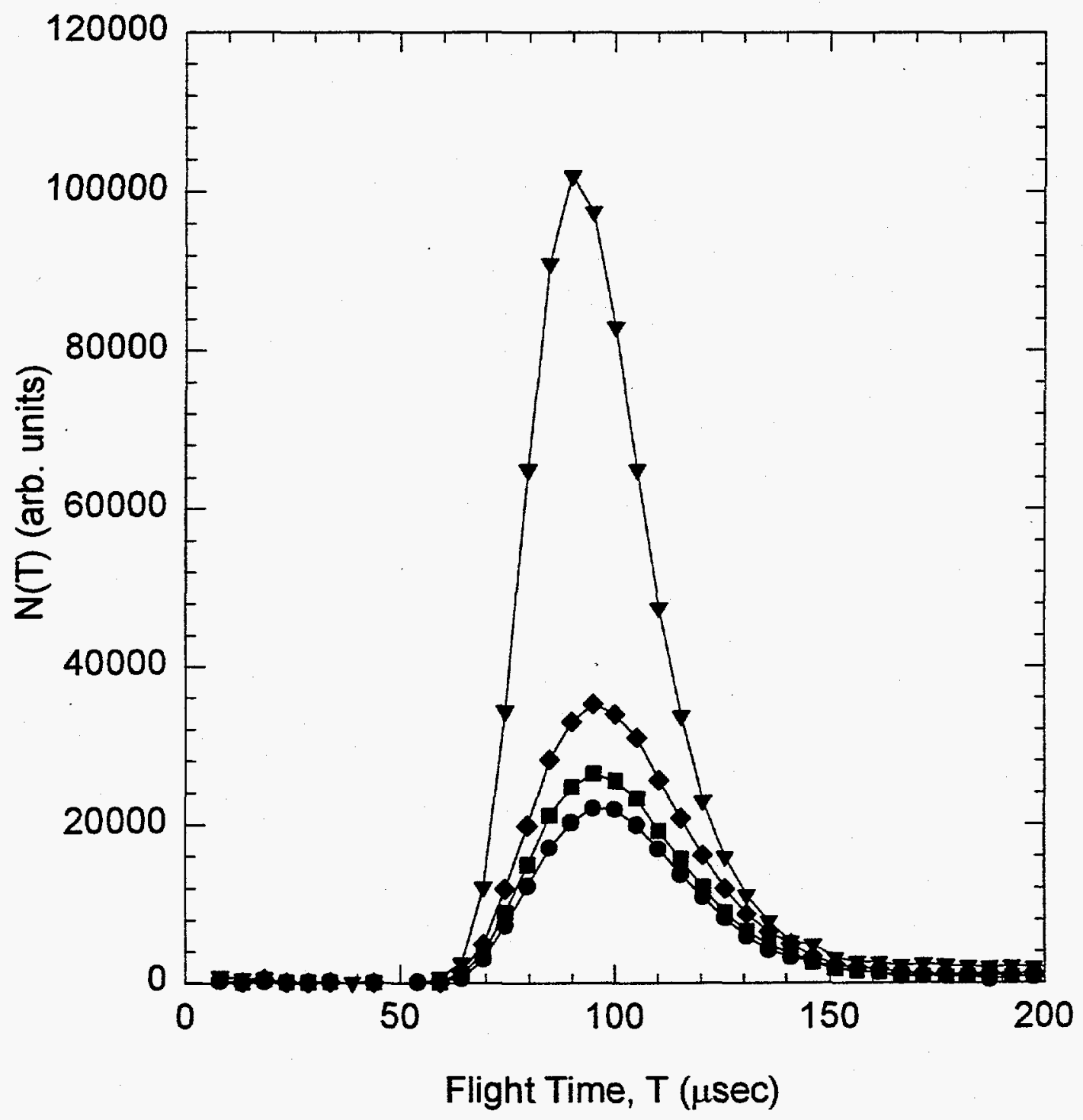

Figure 2-8 


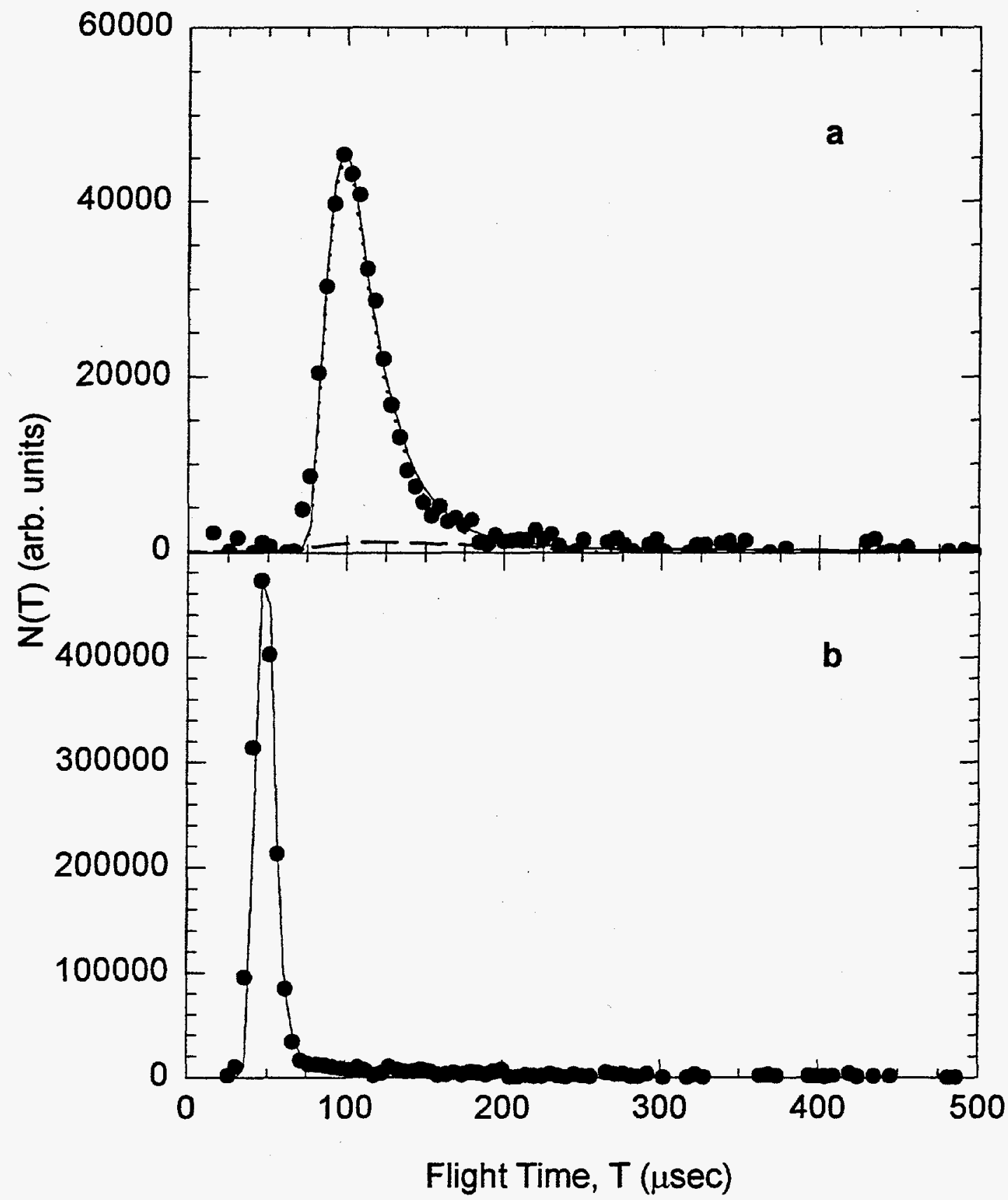

Figure 2-9 


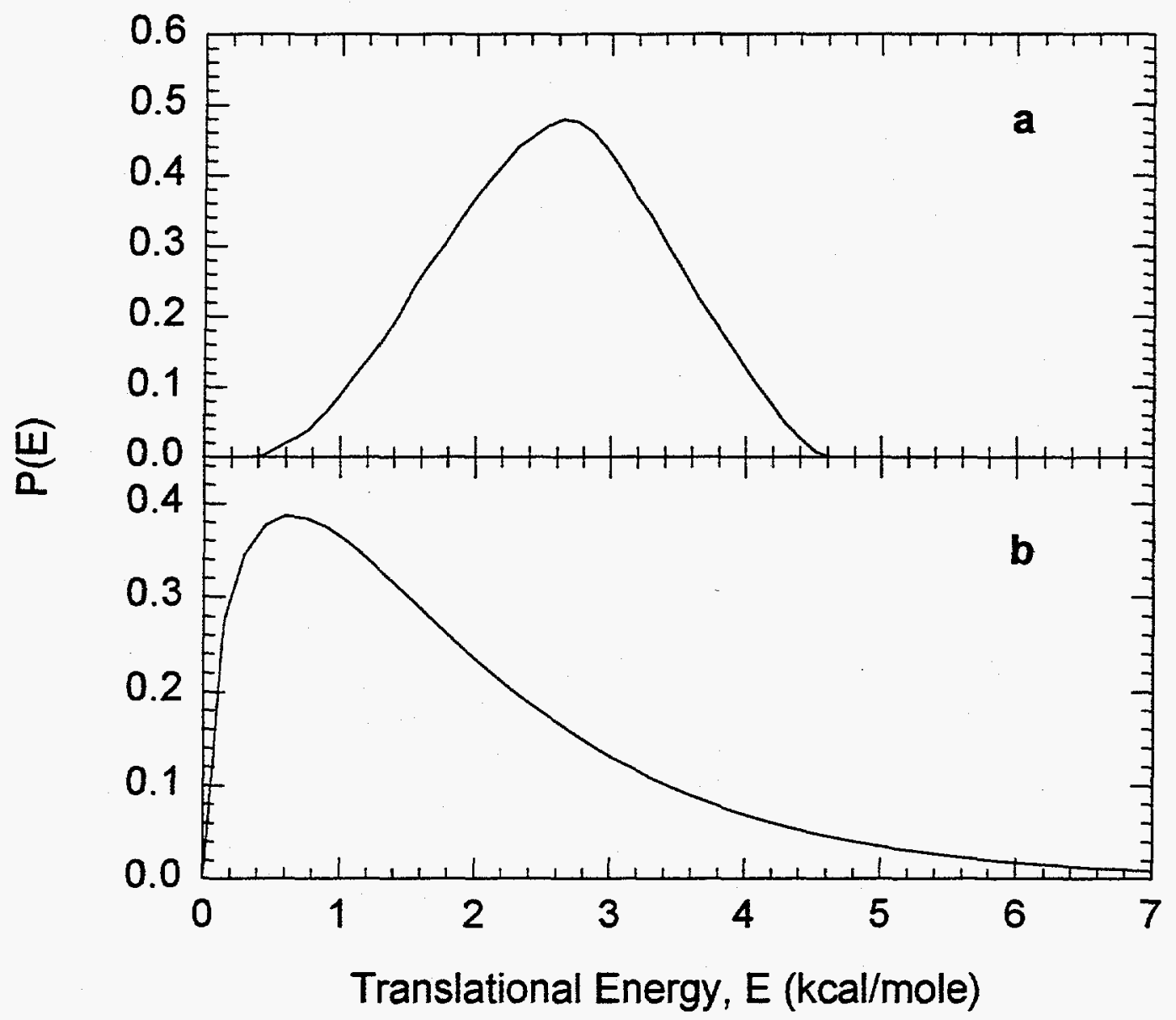

43

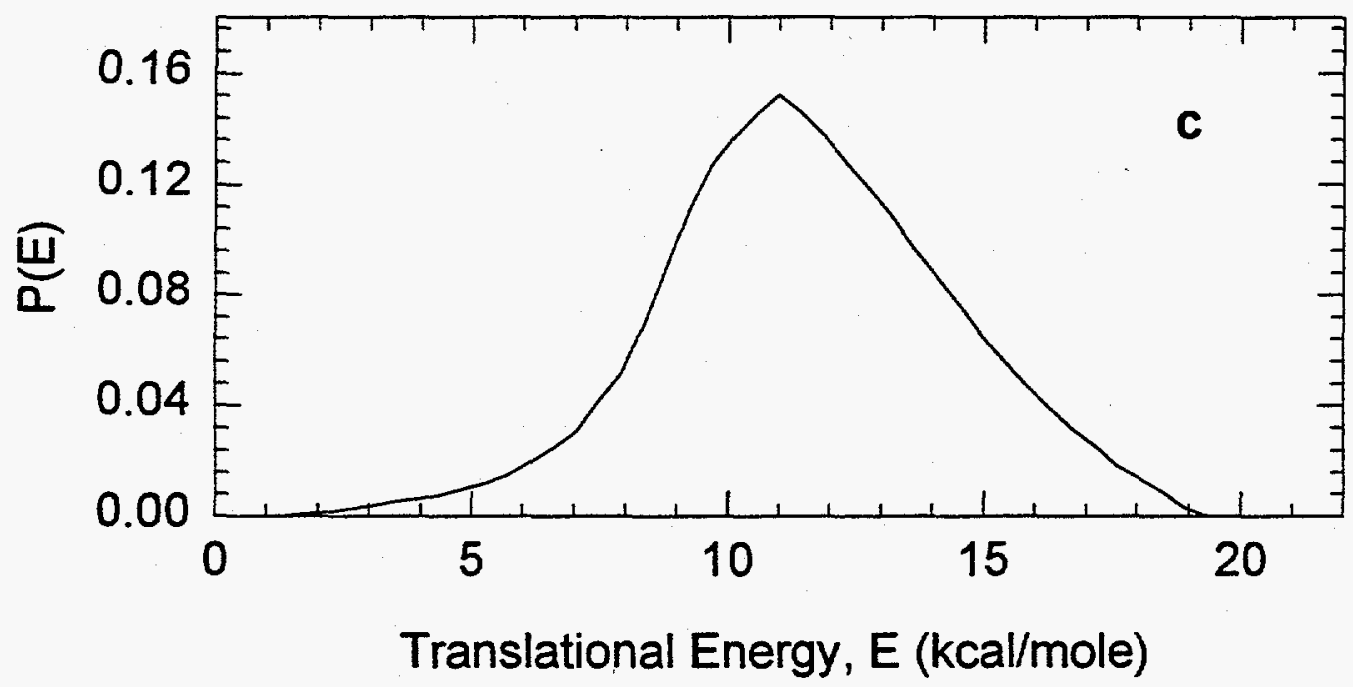

Figure 2-10 

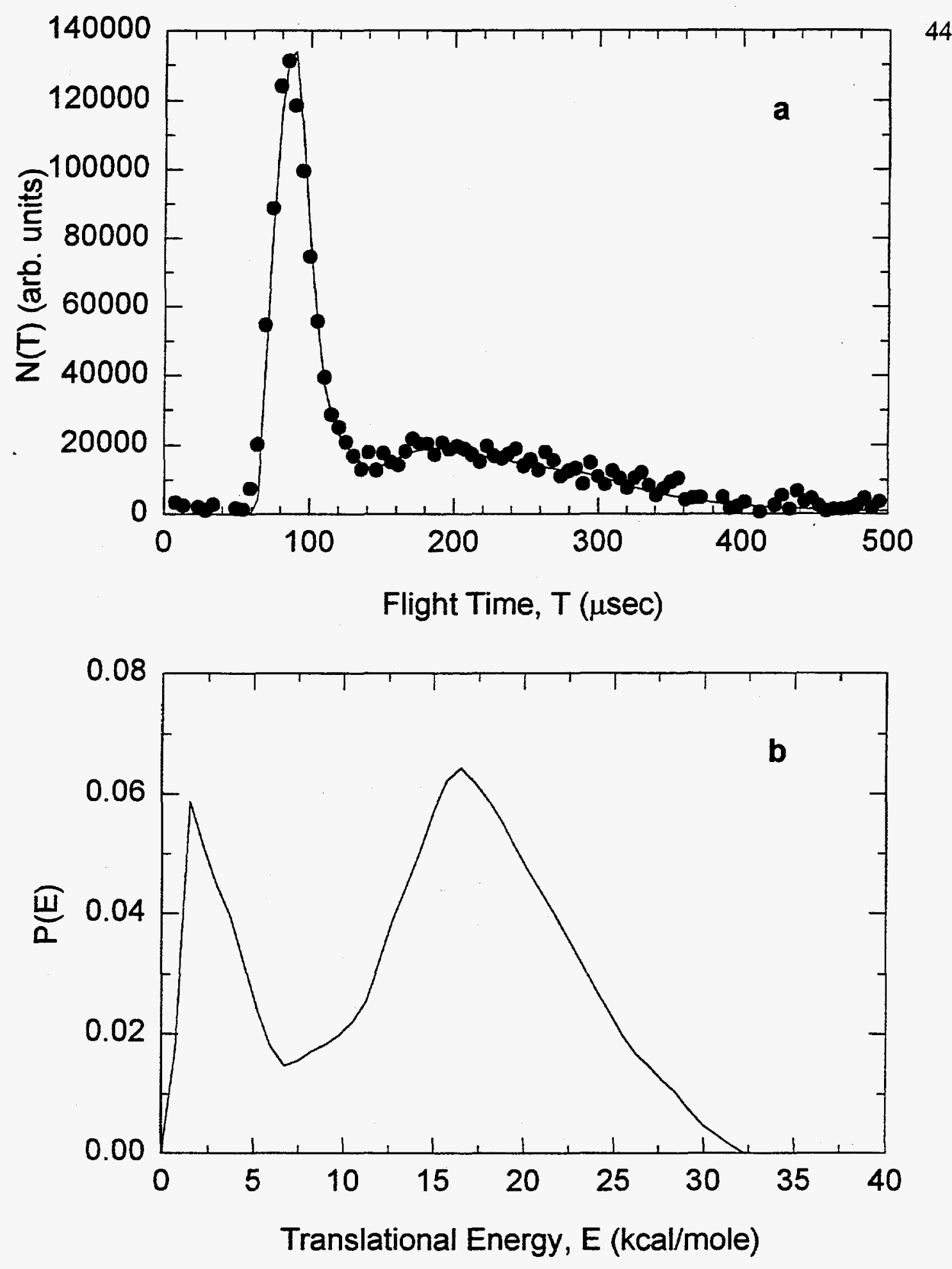

Figure 2-11 


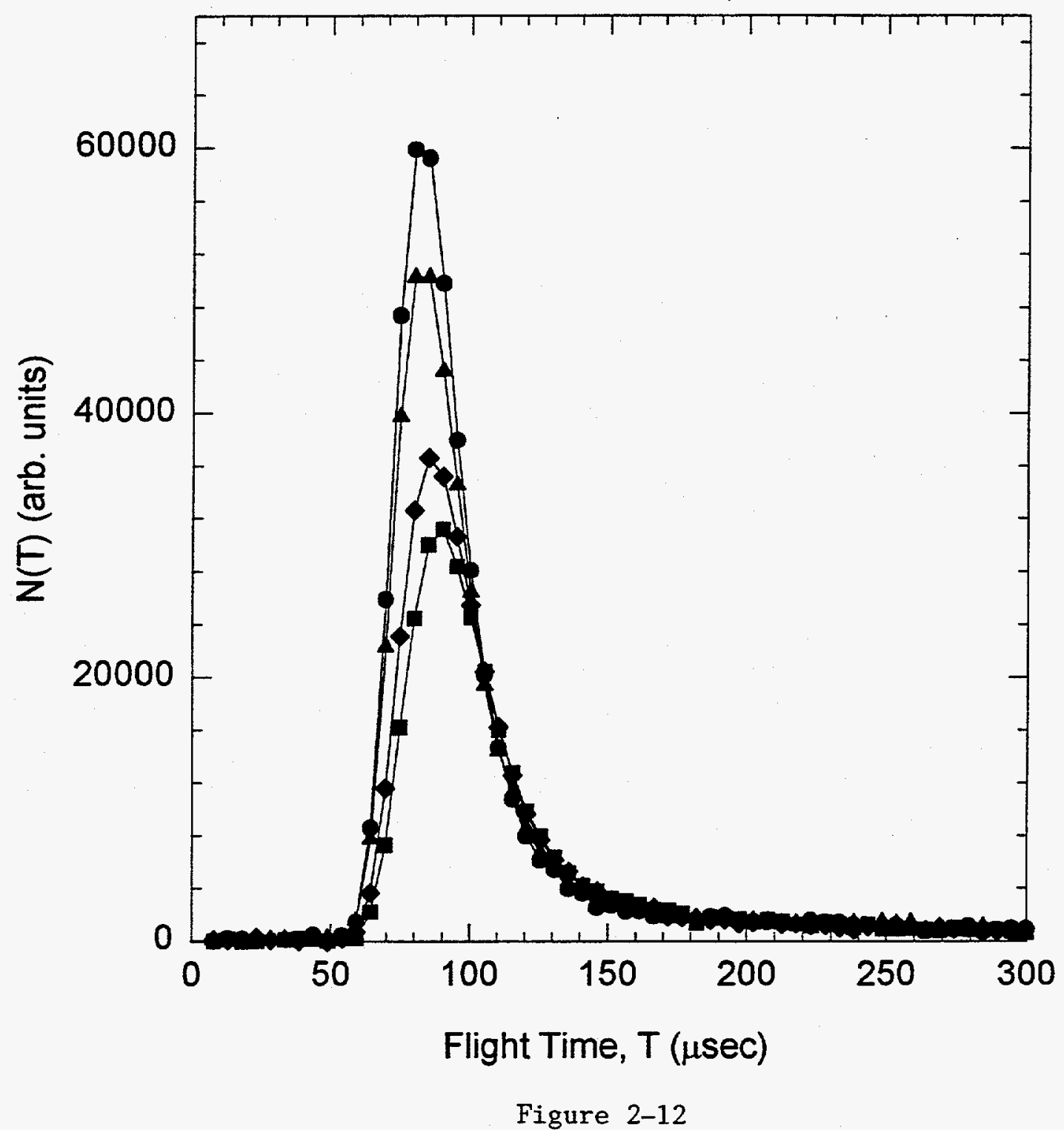




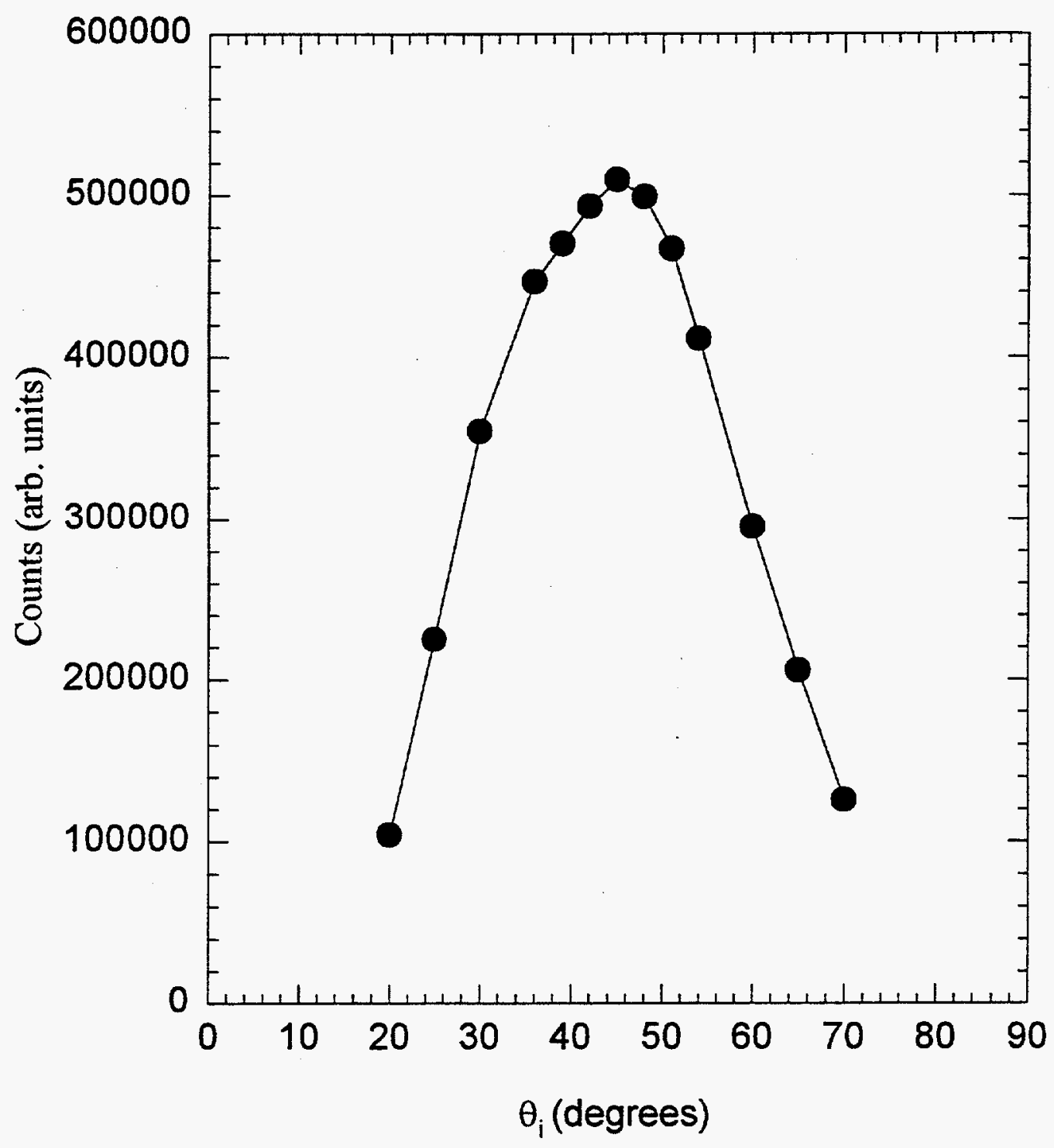

Figure 2-13 

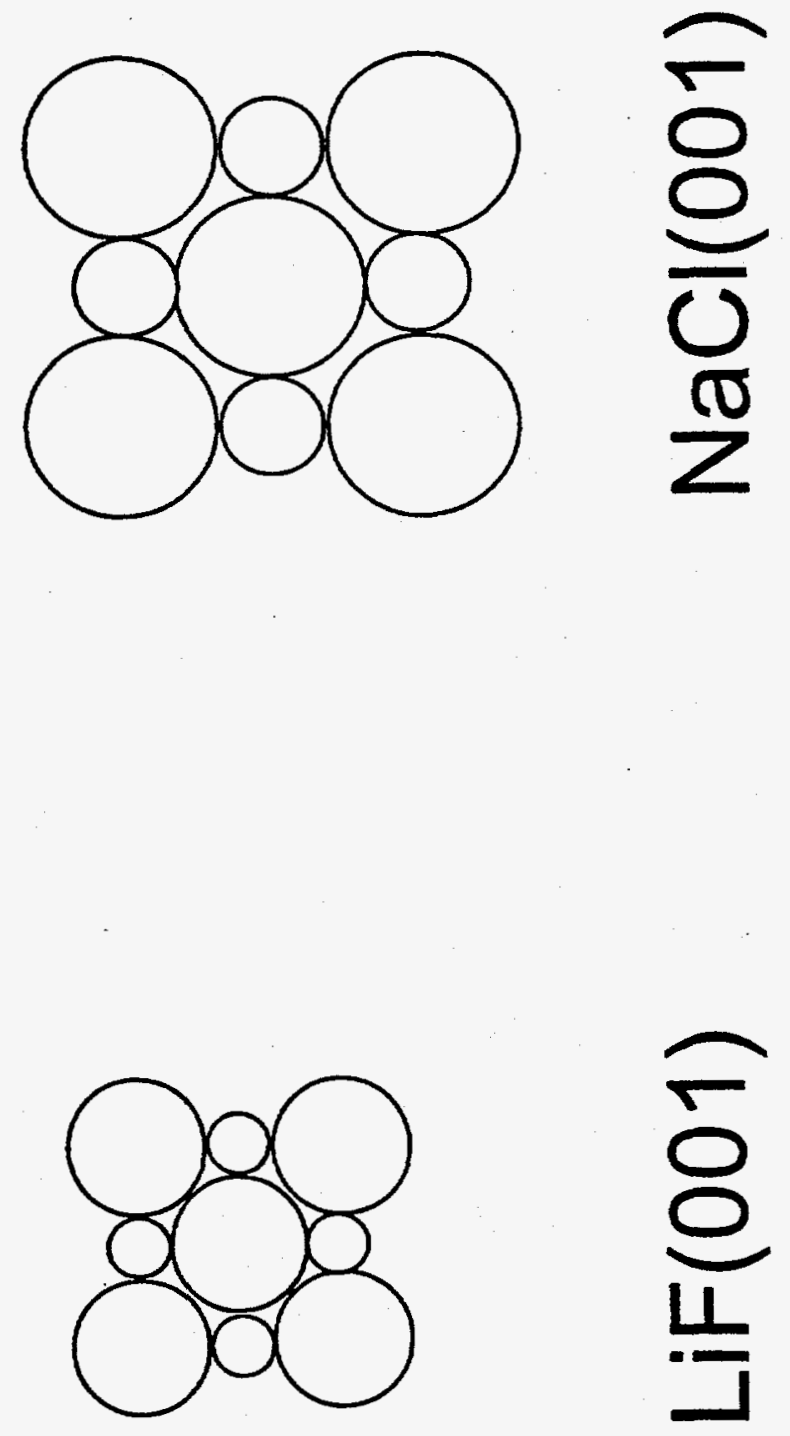

Figure 2-14 


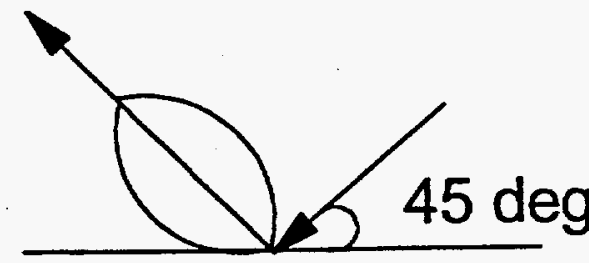

\section{Case 1}

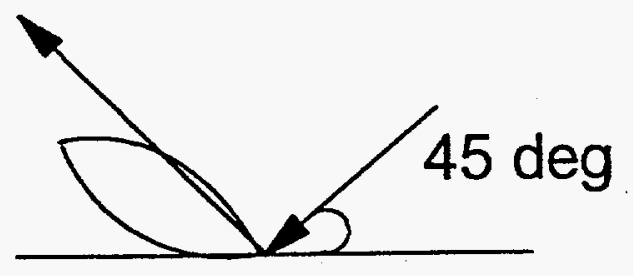

Case 2

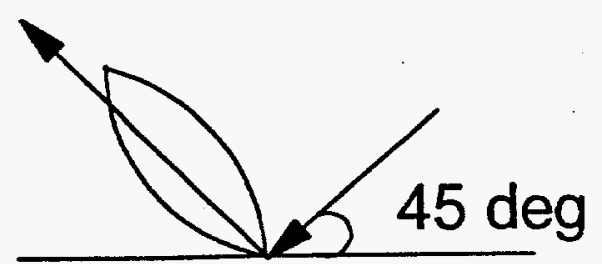

Case 3

Figure 2-15 


\section{Chapter 3}

\section{Energy Transfer in the Scattering of Hyperthermal Rare Gas Beams from a}

\section{CsI Surface}

\section{Introduction}

Energy transfer in hyperthermal scattering of the rare gaseous atoms: Ne, $\mathrm{Ar}, \mathrm{Kr}$, and Xe from a CsI surface has been studied to further understand the mechanisms of energy loss in gas-surface collisions. This is an extension of our work on hyperthermal He atom and Ne atom scattering from $\operatorname{LiF}(001)$ and $\mathrm{NaCl}(001)$. In the hyperthermal energy regime, the masses of the collision partners are expected to be the most important parameter determining the efficiency of energy transfer. In CsI, the mass of Cs is greater than all the rare gaseous atoms used, and thus the condition that $\mu<1$ is always satisified, where

$$
\mu=\frac{M_{g}}{M_{s}},
$$

$\mathrm{M}_{\mathrm{g}}$ is the mass of the gaseous atom, and $\mathrm{M}_{s}$ is the mass of the surface atom involved in the collision. The condition of $\mu<1$ is necessary for the expression for energy transfer in the impulse limit to hold:

where $E_{i}$ is the incident energy. ${ }^{1}$ In addition, for the $I$ anion in CsI, the $M_{I}>M_{N e}$, 


$$
\frac{\Delta E}{E_{1}}=\frac{4 \mu}{(1+\mu)^{2}}
$$

$\mathrm{M}_{\mathrm{Ar}}$, and $\mathrm{M}_{\mathrm{Kr}}$. If impulsive collisions are important, then when the mass of the incoming gaseous atom more closely matches the mass of a surface atom in the CsI crystal, the energy transfer in expected to be more efficient. In Eq. (2), this corresponds to the limit of $\mu=1$, where the fractional energy transfer, $\Delta E / E$, is 1 . In addition, the value of $\Delta \mathrm{E} / \mathrm{E}$ should be independent of the incident collision energy.

As was found in the work on He atom and Ne atom scattering from $\mathrm{LiF}(001)$ and $\mathrm{NaCl}(001)$, simple impulsive collisions do not fully explain the energy transfer observed. Two distinct features appear in the time-of-flight spectra of the scattered atoms in certain ranges of collision energy. This points to more than one mechanism of energy transfer. The trend in energy transfer with mass qualitatively follows the predictions of the impulse approximation, though, and the model is useful in trying to understand the way energy is lost to the surface.

Unlike $\mathrm{LiF}$ and $\mathrm{NaCl}, \mathrm{CsI}$ does not naturally cleave along a lattice plane, and so although a single crystal was used, the surface was rough. A rough surface can lead to more multiple collision energy loss. Also, measuring an angular distribution is less helpful than in the case of $\mathrm{LiF}(001)$ and $\mathrm{NaCl}(001)$ since the surface plane is not as well defined. The angular distributions are so broad that it is often hard to define an angle of maximum flux. In the $\mathrm{LiF}(001)$ and $\mathrm{NaCl}(001)$ scattering, this was useful for determining whether energy was lost preferentially in the directions parallel or perpendicular to the surface. 
Very little experimental work has tested the impulse approximation or explored energy transfer in the hyperthermal regime in a systematic way for neutral particles hitting a surface. Winters et al. studied the collisions of $\mathrm{He}, \mathrm{Ar}$, and $\mathrm{Xe}$ atoms colliding with a Pt(111) surface using supersonic beams, though. ${ }^{2}$ They also studied the ion-surface collisions between $\mathrm{He}^{+}, \mathrm{Ar}^{+}$, and $\mathrm{Xe}^{+}$colliding with a gold film. They report mean final translational energies of the atoms and ions after they scatter from the surface and the fraction of energy lost to the surface, but there are no time-of-flight spectra shown and so presumably distinctive features showing different mechanisms of energy loss were not observed. The energy loss is discussed in terms of binary collisions. Other previous work on hyperthermal scattering has been discussed already in Chapter 2 .

\section{Experimental}

We measured the energy transfer with the same atomic beam surface scattering apparatus used for the $\mathrm{He}$ atom and $\mathrm{Ne}$ atom scattering from $\mathrm{LiF}(001)$ and $\mathrm{NaCl}(001)$ (see Chapter 2). In this experiment, however, a wider range of rare gases and a CsI crystal was used. Seeded beams of $\mathrm{Ne}, \mathrm{Ar}, \mathrm{Kr}$, and $\mathrm{Xe}$ were expanded to form supersonic beams, and each beam was characterized for 1) stagnation pressure, and 2) the power used to heat the nozzle, on a crossed molecular beams apparatus by measuring beam time-of-flight using a spinning slotted wheel as a chopper with the detector looking directly into the beam. The time-of-flight data were fit to the following equation, which is appropriate for a 
supersonic beam:

$$
N(v)=v^{2} \exp \left(-\beta\left(v-v_{0}\right)^{2}\right),
$$

where $\mathrm{N}(\mathrm{v})$ is a number density distribution of speeds; $\beta=\mathrm{m} / 2 \mathrm{kT}$, in $(\mathrm{m} / \mathrm{s})^{-2}$, characterizes the width of the distribution (where $T_{s}$ is the "translational temperature" of the beam); and $\mathrm{v}_{\mathrm{o}}$ is the most probable velocity in the distribution, $N(v)$. The beam energy, which is the collision energy, $E_{\text {coll }}$, in the scattering event, is calculated from $\mathrm{v}_{\mathrm{o}}{ }^{3}$ The range of $\mathrm{E}_{\text {coll }}$ depends on the rare gas used: for $\mathrm{Ne}$ atoms $E_{\text {coll }}=13-63 \mathrm{kcal} / \mathrm{mole}$, for $\mathrm{Ar}$ atoms $\mathrm{E}_{\mathrm{coll}}=10-58 \mathrm{kcal} / \mathrm{mole}$, for $\mathrm{Kr}$ atoms $E_{\text {coll }}=13-73 \mathrm{kcal} / \mathrm{mole}$, and for Xe atoms $E_{\text {coll }}=16-95 \mathrm{kcal} / \mathrm{mole}$. The nozzle stagnation pressure was adjusted to create a good expansion as the temperature of the nozzle was increased. The speed ratio, $\mathrm{S}$, where

$$
S=\nabla_{0} \beta^{\frac{1}{2}}>1
$$

was determined for each mixture at many beam energies. The following speed ratio ranges were obtained: for $0.6 \% \mathrm{Ne} / \mathrm{H}_{2} \mathrm{~S}=6-11$, for $4 \% \mathrm{Ar} / \mathrm{He} \mathrm{S}=7-11$, for $5 \% \mathrm{Kr} / \mathrm{He} \mathrm{S}=8-11$, and for $5 \% \mathrm{Xe} / \mathrm{He} \mathrm{S}=9-14$. The nozzle temperature was determined by measuring beam time-of-flight for a neat $\mathrm{Ne}$ beam and also using optical pyrometry. The beam characterization results are shown in Fig. 1.

The single crystal CsI (Atomergic) was not cleaved because CsI has no natural cleavage plane. A rough surface was used, and the crystal was heated in vacuum for 24 hours at $T_{\text {surf }}=750-800 \mathrm{~K}$. This removes impurities such as water 
and also anneals the crystal. The surface was maintained at $\mathrm{T}_{\text {surf }}=630-640 \mathrm{~K}$ during the experiment to prevent the adsorption of background gases. ${ }^{4}$

As in Chapter 2, the time-of-flight spectra of the $\mathrm{Ne}, \mathrm{Ar}, \mathrm{Kr}$, and $\mathrm{Xe}$ atoms were measured after scattering from the crystal by placing a chopper wheel between the surface and the detector. The flight length was $23.9 \mathrm{~cm}$, and a crosscorrelation chopper wheel was used as before. Time-of-flight spectra were measured for each angle, $\theta_{\mathrm{i}}$, between the incident beam and the surface (see Chapter 2).

\section{Results}

Time-of-flight spectra were measured for fixed collision energies, $E_{\text {coll }}$, and angles $\theta_{\mathrm{i}}$. Each $\theta_{\mathrm{i}}$ distribution was measured with $\mathrm{E}_{\text {coll }}$ held constant. (The dependence on the angle $\phi_{i}$ was not measured since the surface is not a welldefined lattice plane in this case. In the case of $\mathrm{LiF}(001)$ and $\mathrm{NaCl}(001)$, $\phi_{\mathrm{i}}$ was the angle of the incoming beam with respect to the [100] surface direction.)

The time-of-flight spectra of the different rare gases incident with the same collision energy clearly show different energy transfer behavior. In Fig. 2, the time-of-flight spectra of $\mathrm{Ne}, \mathrm{Ar}, \mathrm{Kr}$, and $\mathrm{Xe}$ recoiling from the CsI surface are shown for $E_{\text {coll }}=20 \mathrm{kcal} / \mathrm{mole}$. The time-of-flight spectra reflect the energy that is left over in the gas atom after the collision event. At $E_{\text {coll }}=20 \mathrm{kcal} / \mathrm{mole}$, the Ne and $\mathrm{Ar}$ atom time-of-flight spectra clearly show two components. For the heavier $\mathrm{Kr}$ and Xe atoms, the time-of-flight spectra are broad and the "fast" and "slow" 
peaks are blurred together. The light Ne atoms have lost much less energy than the heavier $\mathrm{Xe}$ atoms, and in addition since $\mathrm{Xe}$ is heavier it moves slower; thus, the time-of-flight peak in Fig. $2 \mathrm{a}$ is at much earlier times than in Fig. 2 d.

For each species, the "fast" and "slow" features in the time-of-flight spectra are blurred together at low $\mathrm{E}_{\text {coll }}$ and become distinct at higher $\mathrm{E}_{\text {coll }}$. The values of $E_{\text {coll }}$ where the peaks become distinct are different for each species. This general trend is illustrated in Fig. 3, where time-of-flight spectra for recoiling Ar atoms are shown for three values of $\mathrm{E}_{\text {coll }}$ : 10,19 , and $48 \mathrm{kcal} / \mathrm{mole}$. Thus, while in Fig. 2 the $\mathrm{Kr}$ and $\mathrm{Xe}$ atom spectra are broad, at higher collision energies, these atoms also show "fast" and "slow" features.

The time-of-flight spectra of $\mathrm{Kr}$ and $\mathrm{Xe}$ atoms for $\mathrm{E}_{\text {coll }}=50$ and $65 \mathrm{kcal} / \mathrm{mole}$, respectively, are shown in Fig. 4 at $\theta_{i}=20^{\circ}$ and $60^{\circ}$. These spectra clearly show "fast" and "slow" components. By taking the spectra at many angles, $\theta_{i}$, the two components can be separated. The lines in Fig. 4 are simulated time-of-flight spectra based on the translational energy distributions shown in Figs. 5 and 6 for $\mathrm{Kr}$ and $\mathrm{Xe}$, respectively. Simulated time-of-flight spectra are generated using the forward convolution method described in Chapter $2 .^{5}$ Simulated spectra are generated for each component and then these are added to get a total fit. In the fitting procedure, the "slow" component of the time-of-flight was assumed to have no variation with $\theta_{i}$, and only the "fast" component was adjusted to get a good total fit at all angles $\theta_{i}$. However, it turned out that very little variation in the "fast" component was required. It is reasonable to fix the simulation for the "slow" 
component at all $\theta_{i}$ since this peak arises from atoms that have lost most of their incident energy. In Fig. 5a, the translational energy distribution used to generate the simulation of the "fast" $\mathrm{Kr}$ atom time-of-flight peak is shown. It is quite broad and extends out to about $34 \mathrm{kcal} / \mathrm{mole}$, which is less than $\mathrm{E}_{\mathrm{coll}}=50 \mathrm{kcal} / \mathrm{mole}$. Thus, no elastic scattering is observed. The "slow" $\mathrm{Kr}$ atom time-of-flight peak is simulated using the translational energy distribution in Fig. 5b. For comparison, a Maxwell-Boltzmann distribution for the surface temperature is also shown. Thus, while these $\mathrm{Kr}$ atoms have lost quite a bit of energy, they have not become completely thermalized to the surface temperature of $646 \mathrm{~K}$. Figs. $6 \mathrm{a}$ and $6 \mathrm{~b}$ similarly show the translational energy distributions for the "fast" and "slow" Xe atoms recoiling after striking the surface with $\mathrm{E}_{\text {coll }}=65 \mathrm{kcal} / \mathrm{mole}$. In the case of $\mathrm{Xe}$, the "slow" atom distribution deviates even more from a Maxwell-Boltzmann distribution than in the case of $\mathrm{Kr}$.

The percentage of the energy transfer, $\% \mathrm{E}_{\text {trans. }}$, based on the collision energy and the peak maximum in the translational energy distribution for recoiling atoms is calculated according to:

$$
\%_{\text {trans. }}=\frac{E_{\text {colI }}-E_{P(X)}}{E_{\text {colI }}}
$$

The results for all the systems studied (taken from spectra measured at $\theta_{i}=45^{\circ}$ ) are shown in Fig. 7. In addition, the $\% \mathrm{E}_{\text {trans. }}$ calculated from Eqn. (2) for different impulsive collisions is indicated at the right side of the graph. In Fig. 7, the $\% \mathrm{E}_{\text {trans. }}$ 
for "fast" and "slow" time-of-flight components is distinguished by the solid and open symbols, respectively.

For the "fast" time-of-flight component, the qualitative variation in $\% \mathrm{E}_{\text {trans. }}$ with the mass of the incoming gas particle follows the expected trend, however the magnitude of $\% \mathrm{E}_{\text {trans. }}$ is not what is predicted by using Eqn. (2), except for the case of $\mathrm{Ne}$ atoms. In addition, the $\% \mathrm{E}_{\text {trans. }}$ for the "fast" peak appears to be independent of $E_{\text {coll }}$, which is also a feature of the impulse approximation model.

For the "slow" time-of-flight component the energy transfer is roughly constant with varying $\mathrm{E}_{\text {coll }}$ and the magnitude of $\% \mathrm{E}_{\text {trans. }}$ for $\mathrm{Kr}$ and $\mathrm{Xe}$ atom scattering is about the same as that predicted using Egn. (2). However, for the case of $\mathrm{Ne}$ and $\mathrm{Ar}$, the $\% \mathrm{E}_{\text {trans. }}$ is much greater than that predicted by the impulse approximation in Eqn. (2).

\section{Discussion}

The hyperthermal scattering results reflect the dynamics of the gas-surface interaction at the repulsive wall of the potential energy surface. The energy transfer is mechanical in nature since no chemical reactions are taking place. It is important to investigate the purely mechanical energy transfer in order to better understand reactive collisions, whose dynamics reflect both mechanical and chemical effects. The applicability of binary collision models can be well tested with rare gases scattering from CsI because the whole range of rare gases can be used while maintaining the $\mu<1$ condition (see Eqn. 1). ${ }^{6}$ In Chapter 2, the 
expression for energy transfer in the impulsive limit for a gas-surface collision was derived by starting from the gas phase version and fixing one mass of the diatomic molecule to be $\infty$. However, Eqn. (2) can also be derived from a model where the incoming particle scatters from a linear chain. ${ }^{7}$ The model is one-dimensional and gives the energy transfer for a linear binary collision; the energy transfer, given by Eqn. (2) is also known as the 'Baule' formula. ${ }^{8}$ The impulse limit is valid when the collision is fast and the gas atom leaves the collision zone before the lattice can respond. Thus, impulsive collisions are expected above a certain threshold of $\mathrm{E}_{\text {coll }}$, where classical mechanics describes the interaction well and the atom can leave faster than the lattice can respond. However, as $E_{\text {coll }}$ increases, the gas atom can also more easily penetrate or deform the lattice, suffering multiple collisions during its outbound motion. Trying to distinguish between these two processes is not always straightforward when looking at the energy transfer, $\% \mathrm{E}_{\text {trans., }}$, resulting from the gas-surface interaction.

There are some factors that reduce the energy transfer given by Eqn. (2). ${ }^{7}$

First of all, the energy transfer will be reduced by increased surface temperature by an amount on the order of $\mathrm{k}_{\mathrm{B}} \mathrm{T}_{\mathrm{s}}$. Including a "thermal average" for the initial motion of the surface atom at a surface temperature, $T_{s}$, results in a modified expression for the energy transfer:

$$
\Delta E\left(T_{s}\right)=\frac{4 \mu}{(1+\mu)^{2}}\left(E_{t}-k_{B} T_{s}\right)
$$


However, for $T_{s} \sim 650 \mathrm{~K}$ in these experiments, $k_{B} T_{s} \sim 1.3 \mathrm{kcal} / \mathrm{mole}$, which is insignificant compared to $E_{i}$. Another factor that reduces the energy transfer from the predictions of Eqn. (2) is averaging over impact parameter to account for a three-dimensional picture (the model was strictly one-dimensional). Thus, the reduction in energy transfer for $\mathrm{Ar}, \mathrm{Kr}$, and Xe atoms (from the "fast" time-offlight peak) compared with the predictions from Eqn. (2) is not surprising. These collisions may indeed be impulsive since the energy transfer exhibits the mass trend and independence of $\mathrm{E}_{\text {coll }}$ predicted by the model.

For the "fast" time-of-flight peak, the energy transfer can also reflect scattering from a higher effective mass, $M_{\text {eff, }}$ at the surface. ${ }^{2,9} M_{\text {eff }}$ replaces $M_{s}$ in Eqn. (1). The following $\mathrm{M}_{\text {eff }}$ values can be calculated for $\mathrm{Ar}, \mathrm{Kr}$, and $\mathrm{Xe}$ atom scattering, respectively: 235,275 , and 364 . These numbers reflect the extent of coupling between surface atoms in the collision, but do not tell exactly how many Cs or I atoms are involved. They can only give a relative picture where Xe scatters from a larger coupled set of atoms than $\mathrm{Kr}$ or Ar. The "fast" peak from $\mathrm{Ne}$ atom scattering gives a $\% \mathrm{E}_{\text {trans. }}$ close to that predicted by Eqn. (2).

The "slow" time-of-flight peak results from very efficient energy transfer, but may result from multiple collisions, depending on the particular system (see Fig. 7). For $\mathrm{Kr}$ and $\mathrm{Xe}$ atom scattering, large values of $\% \mathrm{E}_{\text {trans. }}$ are expected for impulsive collisions, but for Ar and Ne atom scattering, the $\% \mathrm{E}_{\text {trans. }}$ is much greater than that predicted by Eqn. (2). The "slow" time-of-flight peak for $\mathrm{Kr}$ and $\mathrm{Xe}$ 
atoms recoiling from the surface was fit with a translational energy distribution "hotter" than a Maxwell-Boltzmann distribution for the surface temperature, $T_{s}$ (Figs. 5 and 6). For $\mathrm{Kr}$ and $\mathrm{Xe}$ atoms, with $\mathrm{M}_{\mathrm{g}}$ so close to $\mathrm{M}_{\mathrm{s}}$, very efficient energy transfer is expected and so only a few collisions would be needed for complete thermal accomodation. The atoms would then emerge with a MaxwellBoltzmann distribution. Since this is clearly not the case, single collision energy transfer may indeed be taking place for $\mathrm{Kr}$ and $\mathrm{Xe}$ atoms. Thus, for $\mathrm{Kr}$ and $\mathrm{Xe}$ atoms, the "slow" and "fast" time-of-flight peaks may both arise from single collisions, but the nature of the collision partner at the surface may be different. In the case of the "fast" time-of-flight peak, the scattering may be from a coupled set of atoms while in the case of the "slow" time-of-flight peak, a head on collision with a single $\mathrm{Cs}$ or I may be taking place. Unlike the cases of $\mathrm{LiF}$ or $\mathrm{NaCl}$, in $\mathrm{CsI}$ head-on collisions with either the $\mathrm{Cs}^{+}$or $\mathrm{I}^{-}$ions are possible since the cations do not eclipse the anions to the same extent $\left(\mathrm{r}_{\mathrm{Cs}}=1.455 \AA\right.$ and $\mathrm{r}_{\mathrm{I}}=1.785 \AA$ ).$^{10}$

For $\mathrm{Ne}$ and $\mathrm{Ar}$ atom scattering, the energy loss observed as the "slow" timeof-flight peak is likely due to multiple collisions. By successive calculations with Eqn. (2), the energy loss from sequential collisions can be calculated. For successive Ne-Cs or Ne-I collisions, the energy loss observed in Fig. 7 can result from 4-6 collisions. For the case of Ar, the energy loss corresponds to about 2-3 collisions. This kind of energy loss may be the result of the gas atom deforming or penetrating the lattice and then sufferring multiple collisions along its exit trajectory. 


\section{Conclusions}

The hyperthermal scattering of $\mathrm{Ne}, \mathrm{Ar}, \mathrm{Kr}$, and $\mathrm{Xe}$ was investigated to further explore the mass dependence of the gas atom on the efficiency of energy transfer in gas-surface collisions. By measuring the time-of-flight of the recoiling atom as it emerges from the collision zone, the dynamics of the energy loss processes taking place can be examined in detail. For Ne atoms in the range of $E_{\text {coll }}=13-63 \mathrm{kcal} / \mathrm{mole}$ and $\mathrm{Ar}$ atoms in the range of $E_{\text {coll }}=10-58 \mathrm{kcal} / \mathrm{mole}$, one mechanism of energy loss due to multiple collisions and another likely resulting from single collisions were observed. For $\mathrm{Kr}$ and $\mathrm{Xe}$ atoms in the ranges of $E_{\text {coll }}=13-73 \mathrm{kcal} / \mathrm{mole}$ and $16-95 \mathrm{kcal} / \mathrm{mole}$, respectively, two energy loss mechanisms were observed, which may be due to different types of single collision events at the surface. 


\section{References and Notes}

1. R. W. Zwanzig, J. Chem. Phys. 32, 1173 (1960).

2. H. F. Winters, H. Coufal, C. T. Rettner, and D. S. Bethune, Phys. Rev. B 41, 6240 (1990).

3. H. Haberland, U. Buck, and M. Tolle, Rev. Sci. Instrum. 56, 1714 (1985).

4. The melting point of CsI is $899 \mathrm{~K} ; \mathrm{R}$. C. Weast, CRC Handbook of Chemistry and Physics (CRC Press, Boca Raton, Florida, 1989).

5. A. M. Wodtke, Ph. D. Thesis, University of California, Berkeley, 1986;

X. Zhao, Ph. D. Thesis, University of California, Berkeley, 1988; J. D. Myers, Ph. D. Thesis, University of California, Berkeley, 1993.

6. When $\mu>1$, the gas atom is not stopped by the collision and thus penetrates the lattice, suffering multiple collisions. This violates the definition of impulsive scattering since the lattice responds on the time scale of the collision in this case.

7. J. Harris, Dynamics of Gas-Surface Interactions (The Royal Society of Chemistry, London, 1991).

8. F. O. Goodman and H. Y. Wachman, Dynamics of Gas-Surface Scattering (Academic Press, New York, 1976).

9. G. Carter and J. S. Colligon, Ion Bombardment of Solids (Elsevier, New York, 1968). 
10. J. S. Blakemore, Solid State Physics, 2nd Ed. (Cambridge University

Press, Cambridge, 1985) p.17. 


\section{Figure Captions}

Fig. 3-1 Characterization of seeded beams using the high temperature rhenium nozzle. Nozzle temperature calibrated with a neat Ne beam (open squares). $\mathrm{E}_{\text {beam }}$ determined for $0.6 \% \mathrm{Ne} / \mathrm{H}_{2}$ (open diamonds), $4 \% \mathrm{Ar} / \mathrm{He}$ (solid squares), $5 \% \mathrm{Kr} / \mathrm{He}$ (solid triangles), and $5 \% \mathrm{Xe} / \mathrm{He}$ (open circles).

Fig. 3-2 Time-of-flight spectra of (a) Ne, (b) Ar, (c) Kr, and (d) Xe that had $\mathrm{E}_{\text {coll }}=20 \mathrm{kcal} / \mathrm{mole}$ initially (all taken at $\theta_{\mathrm{i}}=45^{\circ}$ ). The time-of-flight spectra reflect the amount of kinetic energy left over in the gaseous atom after collision with the surface.

Fig. 3-3 Time-of-flight spectra for Ar atoms with different incident energies recoiling from CsI (measured at $\theta_{\mathrm{i}}=45^{\circ}$ ). In (a) $\mathrm{E}_{\mathrm{coll}}=10 \mathrm{kcal} / \mathrm{mole}$, in (b) $E_{\text {coll }}=19 \mathrm{kcal} / \mathrm{mole}$, and in (c) $E_{\text {coll }}=48 \mathrm{kcal} /$ mole. The two features in the time-of-flight spectra become clearly separated as $E_{\text {coll }}$ is increased for all the rare gaseous atoms studied.

Fig. 3-4 Time-of-flight spectra for: $\mathrm{Kr}$ atoms with $\mathrm{E}_{\text {coll }}=50 \mathrm{kcal} / \mathrm{mole}$ taken at (a) $\theta_{\mathrm{i}}=20^{\circ}$ and (b) $\theta_{\mathrm{i}}=60^{\circ}$ and Xe atoms with $\mathrm{E}_{\text {coll }}=65 \mathrm{kcal} / \mathrm{mole}$ taken at (c) $\theta_{\mathrm{i}}=20^{\circ}$ and (d) $\theta_{\mathrm{i}}=60^{\circ}$. The fits are generated from the translational energy distributions in Figs. 5 and 6 for $\mathrm{Kr}$ and Xe, respectively. The 
"fast" time-of-flight peak is simulated using the distribution in Fig. $5 \mathrm{a}(\mathrm{Kr})$ or Fig. 6a (Xe) (dotted line), and the "slow" time-of-flight peak is simulated using the distribution in Fig. $5 \mathrm{~b}(\mathrm{Kr})$ or Fig. $6 \mathrm{~b}(\mathrm{Xe})$ (dashed line). The total fit (solid line) is the sum of the "fast" and "slow" contributions.

Fig. 3-5 Translational energy distributions used to fit the $\mathrm{Kr}$ atom scattering data in Figs. 4a and 4b: (a) for the fit of the "fast" time-of-flight peak and (b) for the fit of the "slow" time of flight peak (dashed line). A MaxwellBoltzmann distribution for $\mathrm{T}_{\mathrm{surf}}=646 \mathrm{~K}$ is also shown (solid line) in (b).

Fig. 3-6 Translational energy distributions used to fit the Xe atom scattering data in Figs. $4 c$ and $4 d$ : (a) for the fit of the "fast" time-of-flight peak and (b) for the fit of the "slow" time of flight peak (dashed line). A MaxwellBoltzmann distribution for $\mathrm{T}_{\text {surf }}=648 \mathrm{~K}$ is also shown (solid line) in (b).

Fig. 3-7 Summary of $\% \mathrm{E}_{\text {trans. }}$ for all the systems studied over the entire range of $E_{\text {coll }}$ used in the experiments. Solid symbols represent the $\% E_{\text {trans. }}$ from "fast" time-of-flight peaks and open symbols represent the $\% \mathrm{E}_{\text {trans. }}$ from "slow" time-of-flight components as follows: $\mathrm{Ne}$ (squares), Ar (upright triangles), $\mathrm{Kr}$ (diamonds), and $\mathrm{Xe}$ (hexagons). The $\% \mathrm{E}_{\text {trans. }}$ predicted using Eqn. (2) for different impulsive collisions is shown at right (open circles). 


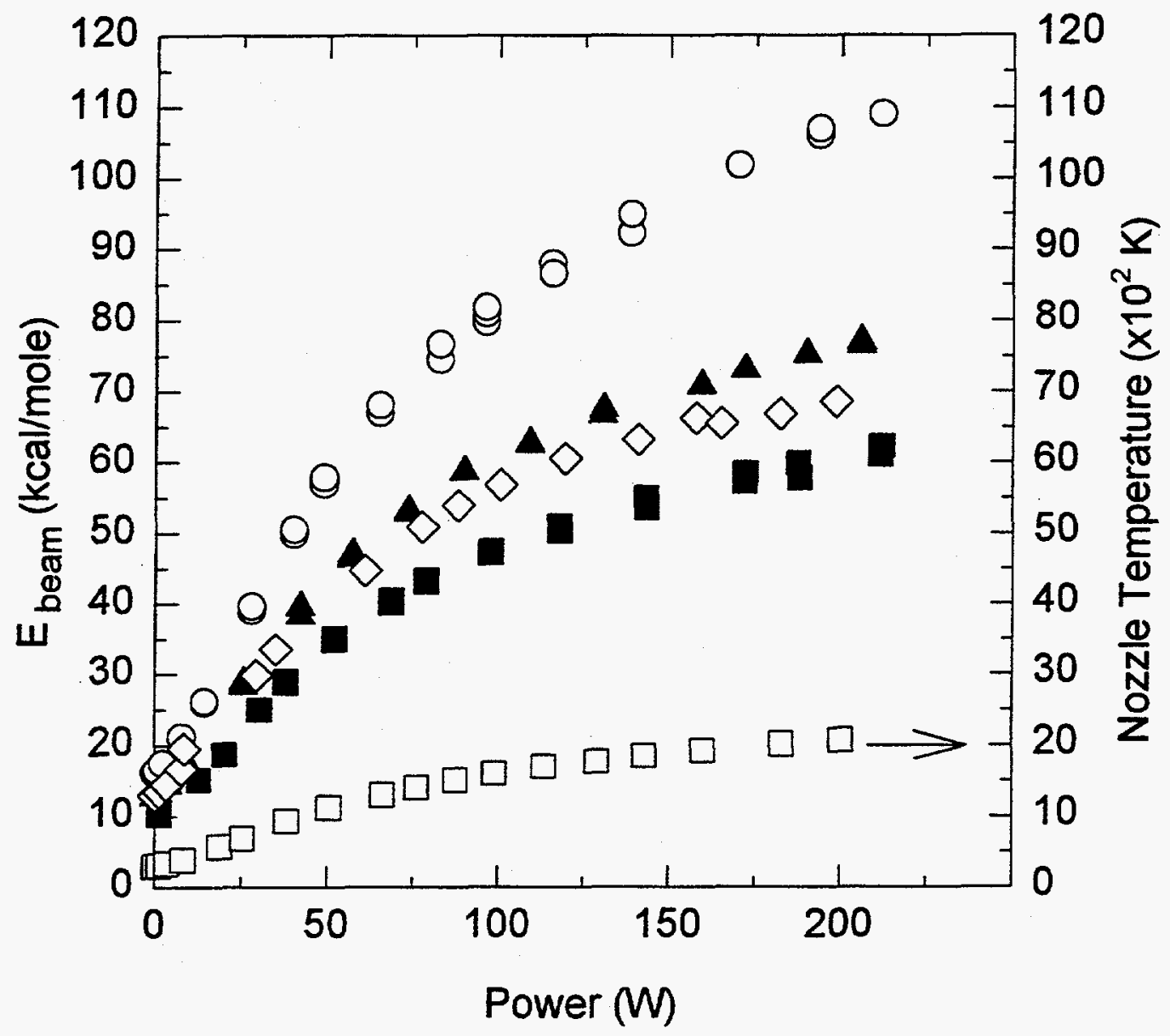

Figure 3-1 


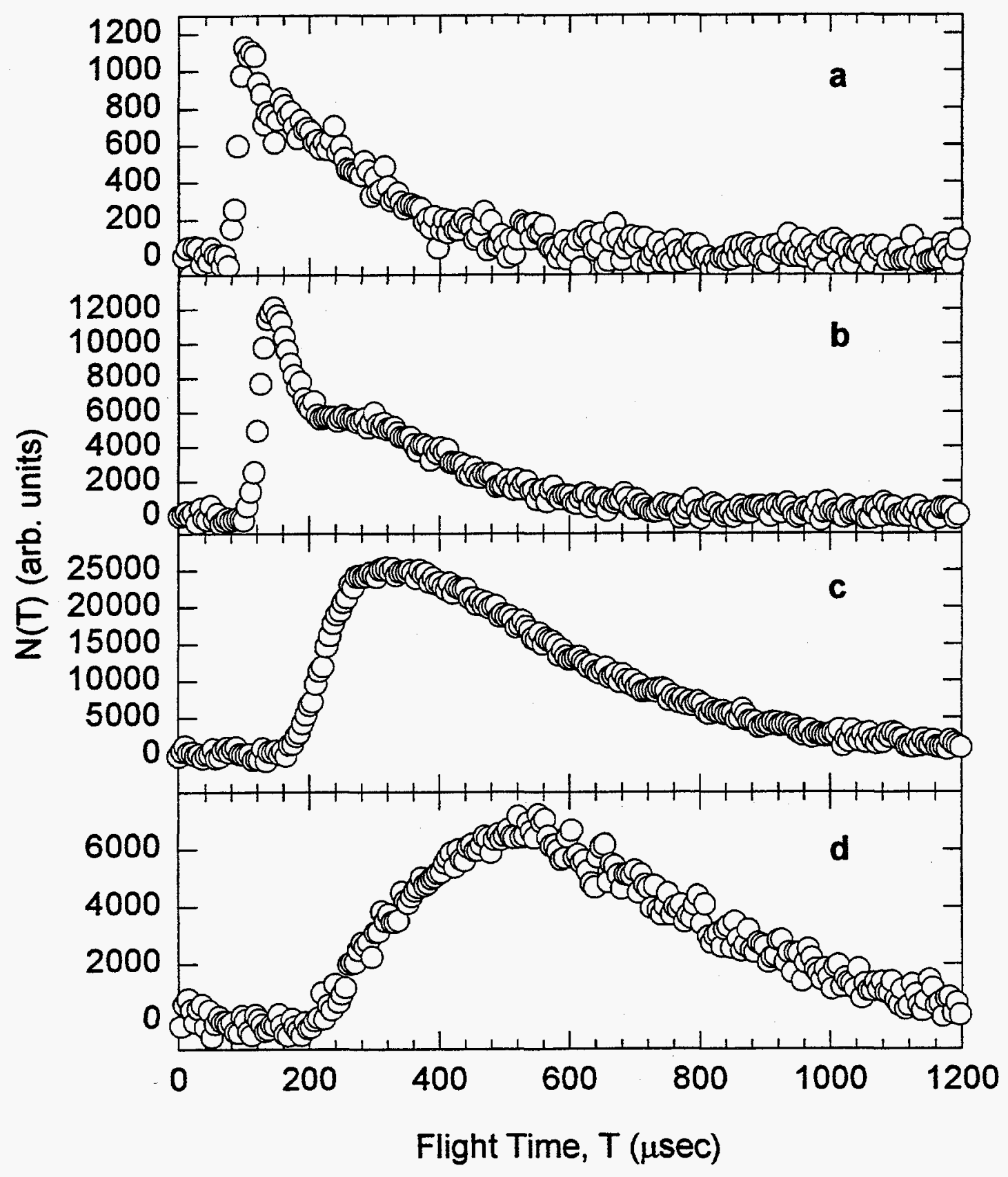

Figure 3-2 


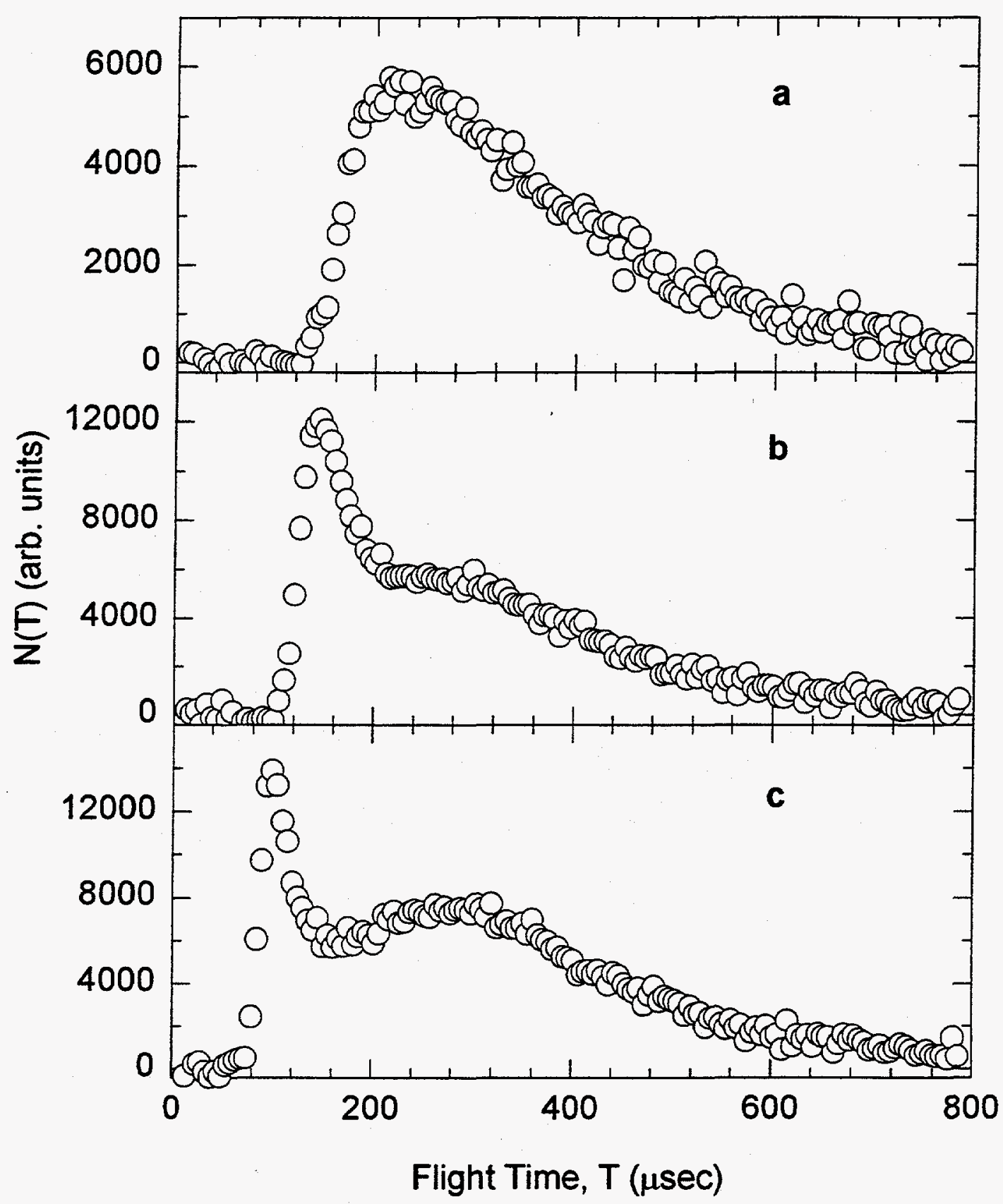

Figure 3-3 


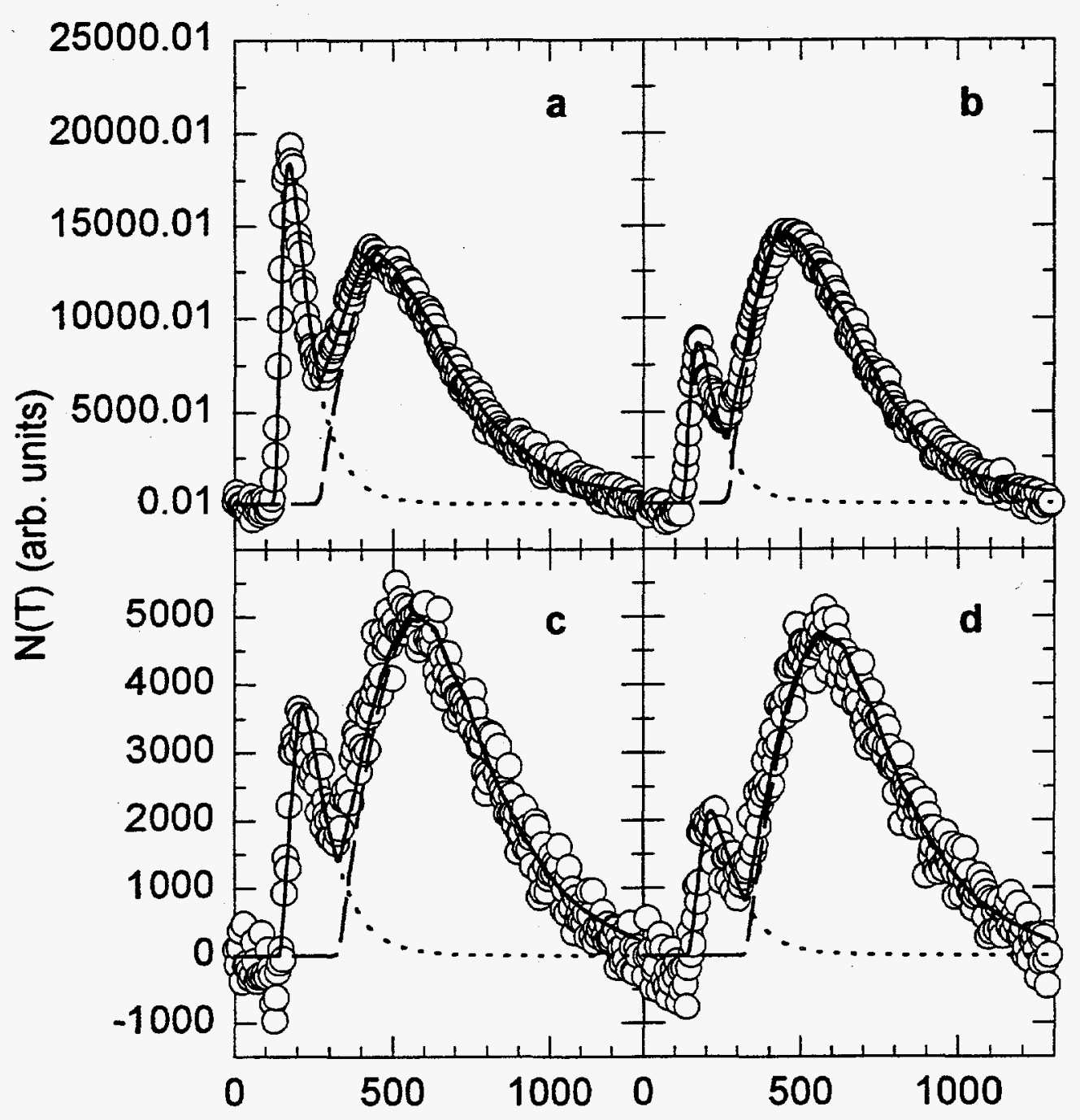

Flight Time, $T$ ( $\mu \mathrm{sec}$ )

Figure 3-4 

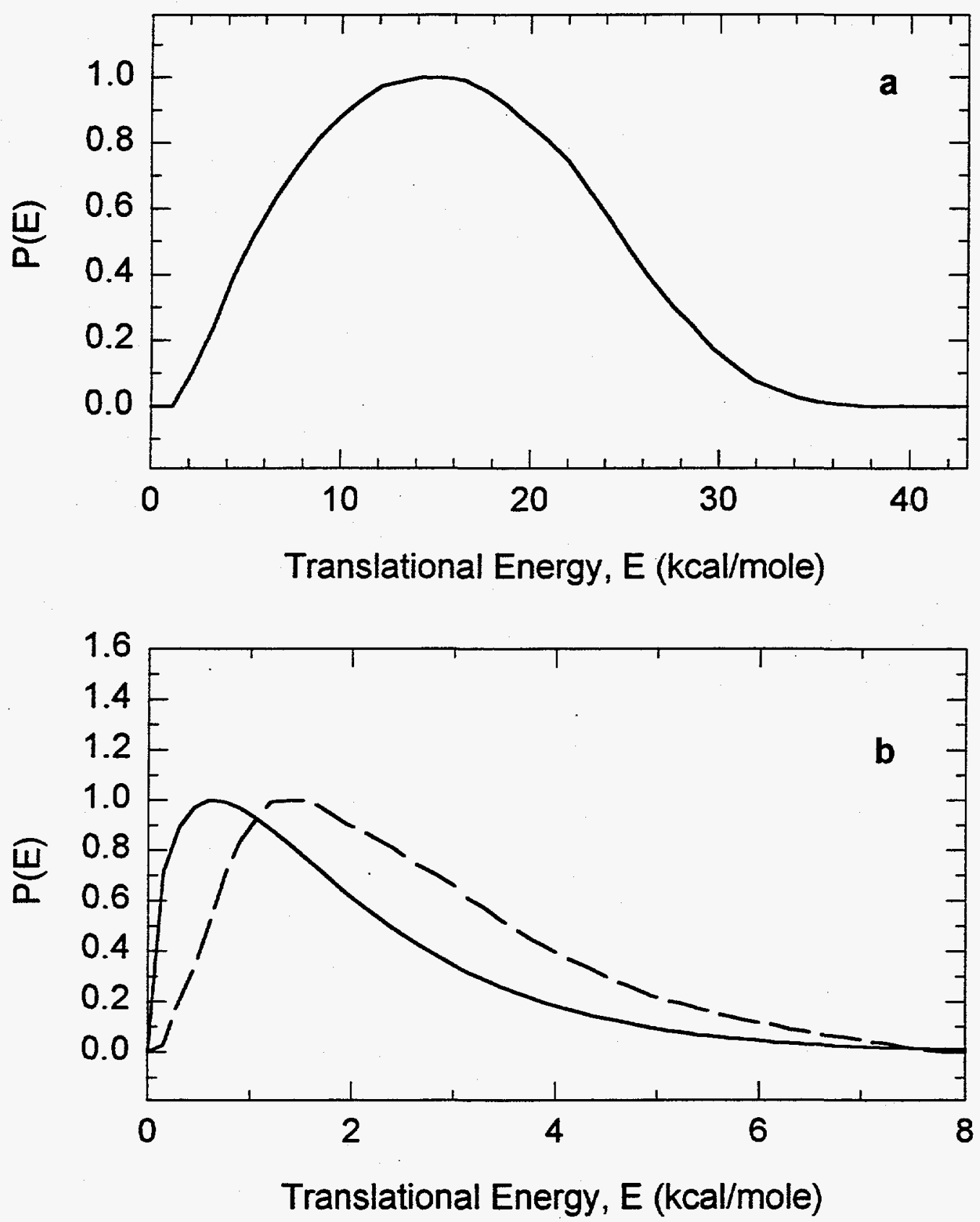

Figure 3-5 

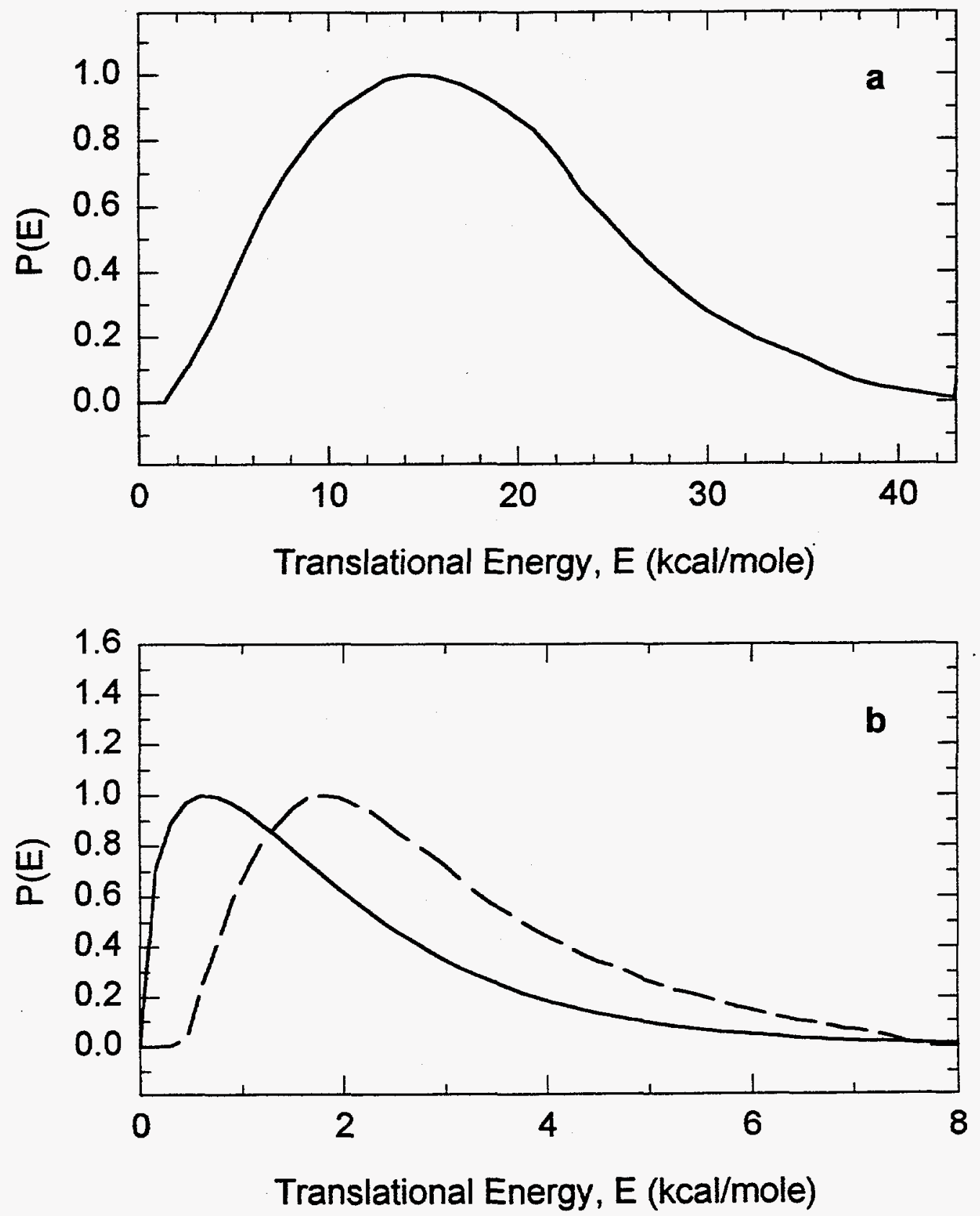

Figure 3-6 


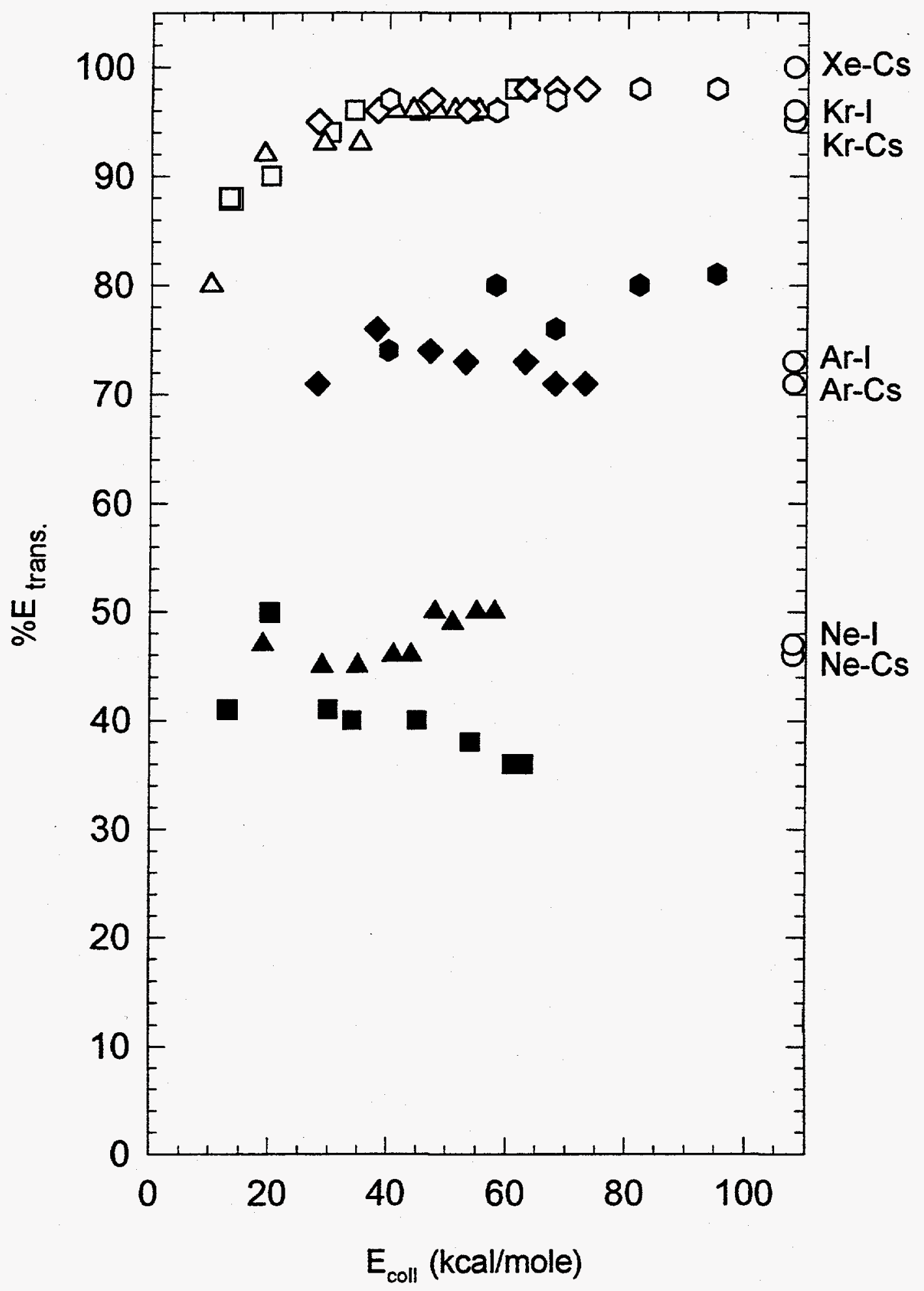

Figure 3-7 


\section{Chapter 4}

\section{Atomic Beam-Surface Scattering Study of HF (DF) Produced in the Reaction: $\mathbf{H}(\mathrm{D})+\mathbf{L i F}(001)$}

\section{Introduction}

Reactions at the gas-surface interface have been successfully probed using molecular beam techniques by many research groups. Modulated beams have been used to study the kinetics of gas-surface reactions by measuring the rates of formation of various product species as they evolve from the surface. ${ }^{1-3}$ The dynamics of the gas-surface reaction can also be studied using molecular beam techniques. Pure adsorption and desorption events, which model important steps in the overall reaction process, have been more extensively studied than reaction product dynamics. ${ }^{4}$ The final step in a gas-surface reaction, where either a bond is formed and/or the new product molecule desorbs from the surface, can be studied by looking at the product translational and/or internal energy as it emerges from the surface. ${ }^{5}$ For example, the work on $\mathrm{D}_{2} \mathrm{O}$ formation in the oxidation of deuterium on $\mathrm{Pt}(111)$ by Ceyer et. al. ${ }^{6}$ shows how the $\mathrm{D}_{2} \mathrm{O}$ product angular and translational energy distributions lead to an understanding of the reaction mechanism. The internal states of product $\mathrm{CO}_{2}$ from $\mathrm{CO}$ oxidation on $\mathrm{Pt}$ have also been measured, showing how different modes, each characterized by its own Boltzmann 
temperature, behave as a function of surface temperature. ${ }^{?}$

In this work, the velocity distributions of individually mass-selected products are measured as they desorb from the surface for different angles between the source, a supersonic atomic beam of $\mathrm{H}$ or $\mathrm{D}$ atoms, and the plane of the surface, $\mathrm{LiF}(001)$, with the detector fixed. In addition to identification of reaction products, analysis of this type of data reveals the dynamical nature of the gas-surface reaction.

Crystalline LiF is usually considered to be a relatively inert material. However, the gas phase reaction $\mathrm{H}+\mathrm{LiF}--->\mathrm{HF}+\mathrm{Li}$ is nearly thermoneutral. ${ }^{8}$ In the solid phase, the LiF lattice commonly contains anion vacancies that can sometimes be filled by $\mathrm{H}$-atoms without too much distortion of the total lattice electron density. ${ }^{9}$ In addition, calculations show that as an $\mathrm{H}$-atom approaches certain types of anion vacancies, a bound state is formed, whereas for $\mathrm{H}_{2}$ the interaction is repulsive. ${ }^{10}$ The interesting dynamics of the $\mathrm{H}$-atom $\operatorname{LiF}(001)$ surface interaction explored here show that $\operatorname{LiF}(001)$ is not inert to $\mathrm{H}$-atoms, and indeed the reaction product HF (and the DF analogue) is directly measured by timeof-flight methods.

\section{Experimental}

A continuous supersonic beam of $\mathrm{H}$ (or $\mathrm{D}$ ) atoms was directed towards a freshly cleaved $\operatorname{LiF}(001)$ surface in the gas-surface scattering apparatus described earlier. ${ }^{11}$ The particles scattered from the surface are chopped by a cross- 
correlation wheel, mass selected, and counted by a channeltron after travelling through a flight distance of $23.9 \mathrm{~cm} .{ }^{12}$ Time-of-flight spectra of individual massselected species are thus measured, which allows for determination of the translational energy imparted to individual products of a gas-surface chemical reaction. In this experiment, the isotopes of $\mathrm{Ne}$ at $\mathrm{m} / \mathrm{e}=20$ and 22 were used to calibrate the mass spectrometer. Also, by using high resolution, $\mathrm{m} / \mathrm{e}=19,20$, and 21 could be clearly distinguished.

The $H$ and $D$ atoms are produced in a supersonic expansion by thermal pyrolysis of neat $\mathrm{H}_{2}$ or $\mathrm{D}_{2}$, using a high temperature nozzle made of pure rhenium metal (Sandvik Rhenium). The nozzle is made of two concentric rhenium tubes that are connected by a rhenium cap, which has been welded to one end under inert atmosphere. A 0.007 " nozzle hole was drilled in the center of the cap by EDM (EDM Exotics). The inner tube is welded to $1 / 4$ " stainless steel tubing, which forms the gas line. Two water-cooled copper clamps, one attached to the inner rhenium tube and one to the outer tube, carry the current and secure the nozzle to a translational stage. The translational stage allows for thermal expansion of the rhenium tubes as they are heated without straining the welds at the cap. The current is thus forced to pass through the welded cap where it can heat the metal to about $2500 \mathrm{~K}$ (using up to $250 \mathrm{~W}$ ). The nozzle temperature has been measured using optical pyrometry and by measuring the beam time-of-flight for a neat $\mathrm{Ne}$ beam. The temperatures from the two measurements agree well (Fig. 1). The nozzle was operated at $2140 \mathrm{~K}$ to produce $\mathrm{H}$ and $\mathrm{D}$ atoms but minimize the 
possibility of evaporating rhenium metal, which becomes a problem above $2500 \mathrm{~K}$. The use of rhenium instead of tungsten, which has been used previously, ${ }^{13}$ was motivated by a desire to find a material that does not become brittle and therefore susceptible to cracking after thermal cycling to high temperatures. The flux of $\mathrm{H}-$ atoms is determined to be $5 \times 10^{18}$ atoms $\mathrm{cm}^{-2} \mathrm{~s}^{-1}$ based on a $3 \%$ dissociation rate of $\mathrm{H}_{2}$ under the beam conditions used.

The LiF samples were cleaved from a single-crystal boule in air along the natural (001) cleavage plane. Freshly cleaved samples were heated in vacuum $(1 \mathrm{x}$ $10^{-7}$ Torr) for $24 \mathrm{hrs}$. at about $750 \mathrm{~K}$ to remove water and anneal the crystal. Experiments were typically run between $650-700 \mathrm{~K}$ to minimize adsorption of impurities. A narrow velocity distribution in the scattering pattern from $\mathrm{H}_{2}$ is a good check of the cleanliness of the surface, and so such time-of-flight measurements were performed before looking for reaction products. The beam was brought to high temperature over the course of $2 \mathrm{hrs}$. and allowed to stabilize, with the crystal out of the beam path. Once the beam was stable, a typical time-offlight spectrum of product HF or DF took about an hour or two to collect, depending on the angle between the beam and the surface plane. The angle $\theta_{\mathrm{i}}$, between the beam and the surface, and $\phi_{\mathrm{i}}$, the angle of the beam with respect to the [100] surface direction, can both be varied independently. No $\phi_{i}$ dependence was observed, though, in the case of HF or DF product evolution, so the spectra in the figures here are all at $\phi_{\mathrm{i}}=0^{\circ}$. The angle $\theta_{\mathrm{i}}$ is defined to be $0^{\circ}$ when the angle between the beam and the surface plane is $0^{\circ}$ (it is the complement of the angle 
between the beam and the surface normal); the detector is fixed at $90^{\circ}$ from the beam.

\section{Results}

$\mathrm{HF}(\mathrm{m} / \mathrm{e}=20)$ and $\mathrm{DF}(\mathrm{m} / \mathrm{e}=21)$ product time-of-flight spectra were measured in separate experiments for different angles, $\theta_{i}$, between the source and the plane of the $\operatorname{LiF}(001)$ surface. The product $\mathrm{DF}$ has the advantage that $\mathrm{m} / \mathrm{e}=21$ has very low background in the mass spectrometer. In addition to monitoring the appropriate product mass, spectra were also taken at $\mathrm{m} / \mathrm{e}=19$, corresponding to fluorine, and also $m / e=1$ or 2 resulting from reflected $H$ and $H_{2}$ or $D$ and $D_{2}$, respectively. The product $m / e=20$ or 21 signal was only seen when the nozzle was hot enough to produce atomic $\mathrm{H}$ or $\mathrm{D}$, and considerable effort was made to keep the experimental conditions the same between HF and DF experiments so that they could be compared. At a nozzle temperature of $2140 \mathrm{~K}$, the maximum $\mathrm{H}$ or $\mathrm{D}$ atom beam energy is calculated to be $\sim 7.5 \mathrm{kcal} / \mathrm{mole}^{14}$

Typical time-of-flight spectra at $\mathrm{m} / \mathrm{e}=20$ for $\mathrm{H}+\mathrm{LiF}(001)$ and at $\mathrm{m} / \mathrm{e}=21$ for $\mathrm{D}+\operatorname{LiF}(001)$ are shown for $\theta_{\mathrm{i}}=30^{\circ}$ along with the time-of-flight for the reflected beam in each case in Fig. 2. The $\mathrm{m} / \mathrm{e}=20$ and 21 time-of-flight spectra show a sharp fast peak and a slower peak. The time-of-flight for the reflected beam in each case has been scaled and shifted to have the same leading edge as the $\mathrm{m} / \mathrm{e}=20$ or 21 data to clearly show that the peak from the beam is narrower. The origin of the fast peak will be addressed in the discussion of the results, but at this 
point suffice it to say that the slow peak is the only real product signal.

At all angles $\theta_{i}$, the HF or DF product time-of-flight is the same as that of $\mathrm{m} / \mathrm{e}=19$, corresponding to $\mathrm{F}$. The matching time-of-flights indicate that $\mathrm{F}$ results from cracking of HF or DF in the ionizer rather than by other mechanisms, such as desorption of $\mathrm{F}$ or $\mathrm{LiF}$ from the surface. Such a desorption mechanism should give rise to a Maxwell-Boltzmann distribution at the surface temperature, which would extend out to longer times in the time-of-flight spectrum. No evidence for this type of mechanism was observed.

Fig. 3 and Fig. 4 show the time-of-flight spectra obtained at $m / e=20$ and $\mathrm{m} / \mathrm{e}=21$, respectively, for different values of $\theta_{\mathrm{i}}$. Also included in the figures are simulated time-of-flight spectra for the slow peak, corresponding to the translational energy distribution in Fig. 5. Each frame has been scaled to show the details of the individual spectra; the change in product yield with $\theta_{\mathrm{i}}$ is shown separately in Fig. 6. The simulated spectra are obtained by the forward convolution method ${ }^{15}$ and include the various apparatus functions that affect a time-of-flight spectrum. In this case, since the fast peak in the product time-of-flight spectra could not simply be removed by subtracting the corresponding reflected beam time-of-flight (due to the different peak widths, see discussion), simulations of the slow peak were done by generating a consistent translational energy distribution at all angles $\theta_{\mathrm{i}^{-}}$For both products, HF and DF, one translational energy distribution that fits the slow timeof-flight peak was found (Fig. 5) with $<E>_{\mathrm{T}}=4.3 \mathrm{kcal} / \mathrm{mole}$ and $\mathrm{E}_{\max }=13$

$\mathrm{kcal} / \mathrm{mole}$. In the region of the peak maximum, the uncertainty is $0.2 \mathrm{kcal} / \mathrm{mole}$, 
but at $\mathrm{E}_{\max }$ (tail of the distribution) the uncertainty is as high as $1.3 \mathrm{kcal} / \mathrm{mole}$. The cross-correlation wheel is limited to $5 \mu \mathrm{sec}$ time resolution, and the energy goes as $E \propto(1 / t)^{2}$, giving rise to greater uncertainties at higher energies.

A $\theta_{\mathrm{i}}$ distribution is calculated by integrating under the curve for the simulated time-of-flight peak in each spectrum. The HF and DF $\theta_{i}$ distributions look similar (Fig. 6), peaking near $\theta_{i}=45-50^{\circ}$. Although these distributions are not full flux angular distributions, given the geometry of the machine, they do indicate that the scattering lobe for the product HF or DF lies near the specular angle.

\section{Discussion}

\section{Atomic Reactant Species}

The fact that HF or DF product is not seen until the beam source nozzle is hot enough to dissociate a significant fraction of $\mathrm{H}_{2}$ or $\mathrm{D}_{2}$ strongly suggests that the atomic species is indeed the reactant in the systems discussed here. Calculations done by Matsumura support this conclusion. ${ }^{16}$ The calculations show that for $\mathrm{H}_{2}$ approaching anion vacancies in the $\mathrm{LiF}$ lattice at either an edge, corner, or surface vacancy, the interaction is highly repulsive. For atomic $\mathrm{H}$, however, there is an attractive interaction, especially when an electron is trapped in the vacancy. $\mathrm{H}_{2}$ approaching a site on the $\operatorname{LiF}(001)$ surface with no vacancy would also not be expected to molecularly adsorb and take part in a reaction. In addition, dissociative chemisorption of $\mathrm{H}_{2}$ is not possible because the dissociative adsorption energy of 1 $\mathrm{eV}$ is smaller than the $2.3 \mathrm{eV}$ necessary to break the $\mathrm{H}-\mathrm{H}$ bond. Therefore, the 
atomic species produced in the beam must be the reactant.

\section{Origin of the Fast Time-of-Flight Peak}

In the time-of-flight spectra of Fig. 3 and Fig. 4, there are clearly two peaks. The fast peak is largest at $\theta_{i}=45^{\circ}$ (not shown), where it completely hides the features of the second, slow peak. As $\theta_{i}$ is moved towards a grazing angle of $10^{\circ}$ or near the surface normal at $80^{\circ}$, the fast peak drops to a minimum and the second, slow peak becomes the dominant feature. The fast peak, when converted to a translational energy distribution, extends out to energies as high as 40 $\mathrm{kcal} / \mathrm{mole}$ for the case of HF. HF coming off the surface with $40 \mathrm{kcal} / \mathrm{mole}$ of translational energy is not physically reasonable, and so other sources of the fast time-of-flight peak must be considered.

In the source, $\mathrm{H}$ or $\mathrm{D}$ atoms are produced, which can be excited in the electron bombardment ionizer to Rydberg states. In fact, even $\mathrm{H}_{2}$ can be a source of excited states of atomic hydrogen in the electron bombardment ionizer. ${ }^{17}$ Such Rydberg $\mathrm{H}$ or $\mathrm{D}$ atoms are energetic neutrals, travelling with the velocity of the beam. As neutrals, they cannot be filtered by the quadrupole, but they can be detected by the channeltron; thus, they show up in spectra taken at $\mathrm{m} / \mathrm{e}=20$ or 21 . In fact, if the fast time-of-flight peak is due solely to Rydberg atoms, it should appear at any mass setting on the quadrupole, and so by tuning to masses not normally expected to produce signal, this hypothesis can be tested. Upon tuning to other m/e settings of the quadrupole, however, the fast peak did not always appear. 
In fact, it only appeared at masses where there was significant background in the mass spectrometer or where the reaction product HF or DF appeared. Thus, Rydberg $\mathrm{H}$ or $\mathrm{D}$ atoms alone cannot fully explain the presence of the fast time-offlight peak.

The fact that the fast time-of-flight peak is observed at masses where background appears offers a clue. $\mathrm{H}^{+}$, metastable $\mathrm{H}_{2}{ }^{18}$ and Rydberg $\mathrm{H}$-atoms (all produced by electron impact) can ionize background gases through collisions just after the ionization region in the detector (and before the quadrupole mass filter). There is a constant source of background because a continuous beam and a crosscorrelation chopper wheel are used in the experiment. Two processes can occur to give rise to signal at background masses. First of all, $\mathrm{H}^{+}$formed in the ionizer can undergo charge exchange collisions that ionize background gas molecules. ${ }^{19}$

Secondly, through processes such as Penning ionization and associative ionization, the background molecules can be ionized by Rydberg $H(D)$ atoms or metastable $\mathrm{H}_{2}\left(\mathrm{D}_{2}\right)$ and therefore mass-selected by the quadrupole. ${ }^{20}$ They appear in the timeof-flight spectrum at the characteristic time of the excited $\mathrm{H}, \mathrm{D}, \mathrm{H}_{2}$, or $\mathrm{D}_{2}$, which is very fast. In comparing the reflected beam time-of-flight with the fast peak due to collisional ionization (Fig. 2) the collisional ionization peak is broader, but it is reasonable that the collisional process would give rise to broadening since the background molecules are moving at random velocities and the collisions occur with a wide range of impact parameters. All of the above mechanisms may be operating simultaneously, but the density of $\mathrm{H}_{2}\left(\mathrm{D}_{2}\right)$ is much greater than $\mathrm{H}$ (D), 
and so charge exchange from the molecular species is probably contributing more to the fast time-of-flight peak than atomic species involved in Penning and associative ionization processes.

In comparing the collisional ionization effect from different spectra, it is interesting to note the intensity dependence of the fast time-of-flight peak on the mass-selected species and the angle $\theta_{\mathrm{i}}$, which determines how much of the incident beam is directly reflected into the detector. In comparing spectra for different mass-selected species, the highest intensity is seen for $\mathrm{m} / \mathrm{e}=28$, which is usually the highest background mass peak in any vacuum system and normally attributed to CO. For $\theta_{i}=45^{\circ}$, the ratio of the fast peak for the $\mathrm{m} / \mathrm{e}=28$ spectra to that of the $\mathrm{m} / \mathrm{e}=21$ (product DF) spectra is $5: 1$. The intensity of the fast peak seen in spectra taken at $\mathrm{m} / \mathrm{e}=18$ is only $4 \%$ of the $\mathrm{m} / \mathrm{e}=28$ peak, which is consistent with a low background due to residual $\mathrm{H}_{2} \mathrm{O}$ in the vacuum. In the $\mathrm{m} / \mathrm{e}=21$ spectra, there is a strong dependence of the collisional ionization peak intensity on the angle $\theta_{\mathrm{i}}$. The intensity of the collisional ionization peak drops by a factor of 4 when changing from $\theta_{i}=30^{\circ}$ to $\theta_{i}=20^{\circ}$, but the intensity of the slow peak due to the products remains the same. The angular dependence shows that the fast time-of-flight peak has a strong dependence on the incident beam, as expected for the collisional ionization mechanism.

The arguments above rule out the possibility of the fast peak arising from reaction products formed at the surface, which is the main interest in this experiment. Attention is thus focused on the slower peak, which is why a fit for 
only the second peaks and the corresponding translational energy distribution is displayed in Figs. 3-5.

\section{Dynamics of HF and DF Products}

The HF and DF reaction products, measured directly as they evolve from the surface, appear as the slower peaks in the time-of-flight spectra of Fig. 3 and Fig. 4, respectively. The translational energy distribution in Fig. 5 is derived by fitting the slow peak for both products at each angle $\theta_{\mathrm{i}}$. The difficulty in this method is finding the true leading edge of the slow peak, which corresponds to the high energy limit of the translational energy distribution. However, at $\theta_{i}=10^{\circ}$, the leading edge is distinguishable since the signal between the first and second peaks drops to the baseline. The same translational energy distribution is used to fit the data from both reactions at all angles $\theta_{\mathrm{i}}$.

The translational energy distribution for HF and DF is not a MaxwellBoltzmann distribution at the surface temperature. Fig. 7 compares the product translational energy distribution from Fig. 5 with a Maxwell-Boltzmann distribution at the surface temperature, $T_{\text {surf }}=690 \mathrm{~K}$. The product distribution peaks at higher energy than a Maxwell-Boltzmann distribution, about $2.5 \mathrm{kcal} / \mathrm{mole}$, with a substantial fraction of the molecules having up to $13 \mathrm{kcal} / \mathrm{mole}$ of translational energy. The translational energy distribution peaking far from the MaxwellBoltzmann distribution peak indicates that there is a barrier in the exit channel. Although it is possible to fit the HF and DF products separately so that they 
each have their own translational energy distribution, such a distinction between the products is not strongly supported by the data. The difference in the energy distributions is too small to be justified given the signal/noise ratio and the added complication of the collisional ionization peak near the leading edge of the product time-of-flight peak. However, it is interesting to note that when two distributions are used, best fit to all the $\theta_{\mathrm{i}}$ for a particular product, the DF appears to have less energy left over in translation than the HF upon separating from the $\operatorname{LiF}(001)$ surface (although the two distributions have the same $E_{\max }$ ). This suggests that $D F$ has more energy in rotational degrees of freedom, which is not surprising considering the rotational constants $\mathrm{B}_{\mathrm{HF}}=20.939 \mathrm{~cm}^{-1}$ and $\mathrm{B}_{\mathrm{DF}}=11.007 \mathrm{~cm}^{-1}$. 21 Only one translational energy distribution is used to fit both the HF and DF data here, though, which is reasonable since the features of the potential energy surface influencing the dynamics should be the same.

If the total available energy for the reaction to make HF or DF were known, then the translational energy distribution would give more detailed information about the internal states of the product molecules. This in turn would reveal some details of the reaction mechanism. However, the energy required to form and remove the $\mathrm{HF}$ or DF molecule is not known. If, for the sake of forming a qualitative picture, one assumes the maximum energy in the translational energy distribution is also the total available energy to the product $\mathrm{HF}$ or DF, and that the products are all in $v=0$, then the rotational states lying under the distribution of Fig. 5 can be as high as $\mathrm{J}=14$ for $\mathrm{HF}$ and $\mathrm{J}=20$ for $\mathrm{DF}^{22}$ Since the vibrational 
spacing for $\mathrm{HF}$ is $11.83 \mathrm{kcal} / \mathrm{mole}$, it is unlikely that any vibrational excitation occurs. For DF, the vibrational spacing is lower, $8.57 \mathrm{kcal} / \mathrm{mole}$, so it might be energetically possible to have some vibrational excitation. The shape of the translational energy distribution does not support this conclusion, though. Qualitatively, the rotational excitation indicates that in the exit trajectory off the surface, the potential exerts some torque on the molecule. However, there are certainly other factors leading to the broadening of the time-of-flight spectrum, so the $J$ values are not a strict assignment. For example, a variety of surface sites leading to reaction will broaden the product distribution.

The fact that HF and DF have the same maximum energy gives a clue about the microscopic picture of the molecule's departure from the surface. Two extreme cases can be considered. First, if the "kick" off the surface is directed to the light end of the diatomic product, the translational energy distributions for $\mathrm{HF}$ and DF, particularly the $E_{\max }$, will be different depending on whether $H$ or $D$ receives the "kick" (Fig. 8). In fact, DF should have about four times the kinetic energy of HF in this scenario (a factor of 2 from $v_{D}{ }^{2}$ and a factor of 2 from $M_{D}$ ). In the second case, however, if the heavy fluorine end gets the "kick" then the translational energy distributions will be about the same for HF and DF, which is what is observed in the experiment. Thus in this picture, if a strong torque is exerted to one end of the product by the potential, it must be applied toward the fluorine rather than the $\mathrm{H}$ or $\mathrm{D}$ end of the diatomic product. However, in the case of $\mathrm{HF}$ and DF the center-of-mass is very close to the fluorine end of the molecule, so if 
the repulsion is applied toward the center-of-mass, a similar $E_{\max }$ would also be observed. It is safe to say, though, that a strong torque is not applied to the light end of the product HF or DF.

The $\theta_{i}$ distributions for HF and DF in Fig. 6 are peaked with their maxima near the specular angle. Fig. 9 shows the experimental $\theta_{i}$ distribution for HF and a cosine distribution. The cosine distribution would be observed if: 1) the desorbing product had no "memory" of the incident beam and 2) the desorbing product molecule was completely in equilibrium with the surface, at the surface temperature of $690 \mathrm{~K}$. Clearly, the two distributions are different. The experimental distribution is highly peaked towards $45-50^{\circ}$ (angle between the detector and the surface normal, which is equal to $\theta_{\mathrm{i}}$ ). A simple fit to a $\cos ^{\mathrm{n}} \theta$ function is not appropriate, however, since $E_{\perp}$ (the component of the beam energy normal to the surface) changes with $\theta_{i}$ (i.e. as the surface is rotated the incident and final angles are changed simultaneously). It can be concluded, however, that the angular distribution has some dependence on the incident atomic beam angle, and hence $E_{\perp}$. Thus, the product molecule does have some "memory" of the incident beam. This indicates that the HF and DF are formed through an Eley-Rideal mechanism, which is fairly unusual. Most gas-surface reactions are of the Langmuir-Hinshelwood type..$^{23-25}$ 


\section{Surface Reaction Mechanism}

The possibility of surface roughening as a result of the reactions with $\mathrm{H}$ and D atoms was investigated. The $\mathrm{H}_{2}$ or $\mathrm{D}_{2}$ time-of-flight patterns from the reflected beam should be sensitive to surface roughness. A less well-ordered surface will give a broader time-of-flight pattern. However, comparison of HF or DF time-offlight spectra before and after 3 hours of reaction at the same spot on the surface showed no evidence for surface roughening.

The nature of the surface site in this reaction is unknown. The $\operatorname{LiF}(001)$ surface has steps and terraces as well as anion vacancies. The vacancies may have an electron trapped in them. The different sites are expected to have different reactivities. Matsumura's calculations ${ }^{26}$ clearly show that for anion vacancies, depending on the type (with or without a trapped electron) and location (i.e. corner, edge, or surface), the $\mathrm{H}$-atom will feel a different potential. For example, the energy for adsorption of an $\mathrm{H}$-atom on a surface vacancy with a trapped electron is as high as $-6.266 \mathrm{eV}$, and the $\mathrm{H}$ sits in the lattice as $\mathrm{H}^{-}$where it mimics the missing $\mathrm{F}^{-}$. On the other hand, an $\mathrm{H}$-atom interacting with the same vacancy without a trapped electron has an adsorption energy of only $-0.062 \mathrm{eV}$, and the potential curve is largely repulsive. Similar differences in the corner and edge sites are reported. Since the HF and DF in this experiment leave the surface with up to 13 $\mathrm{kcal} / \mathrm{mole}$ of translational energy, it seems unlikely that the trapped electron vacancy is the site of reaction. If the $\mathrm{H}$ can sit in the lattice as $\mathrm{H}^{-}$, then formation of HF that leaves with excess kinetic energy should be unfavorable. Also, the F 
that the $\mathrm{H}$ reacts with would have to be either an interstitial $\mathrm{F}$ or one diffusing along the surface, because in a "perfect" lattice the H would only see Li neighbors. More likely, the HF or DF is formed at sites where the H or D atom cannot easily replace $\mathrm{F}$ in the lattice, and then the newly formed molecule feels a repulsive potential as it leaves the surface. The question remains, though, whether $\mathrm{H}$ reacts at an anion vacancy site (without a trapped electron) or creates one by pulling $F$ out of the lattice.

\section{Conclusions}

A supersonic beam of $\mathrm{H}$ or $\mathrm{D}$ atoms incident on a $\mathrm{LiF}(001)$ surface can be used to study the dynamics of the HF or DF product formed. By directly measuring the time-of-flight of the product species as it evolves from the surface, features of the potential energy surface involved are elucidated. The translational energy distribution describing both products peaks higher in energy than a Maxwell-Boltzmann distribution, indicating that there is a barrier in the exit channel for $\mathrm{HF}$ and $\mathrm{DF}$ to form and evolve from the surface. There is also evidence of rotational excitation, and a microscopic picture of the molecule-surface interaction is discussed. However, because the energetics of the reaction are unknown in this case, details about the internal states of the products cannot be resolved. A state-specific study of the reaction products could help to further understand the details of the surface reaction mechanism.

With an ultra-high vacuum system, the reaction could be explored over a 
wide range of surface temperatures. Here, surface temperatures were limited to $650-700 \mathrm{~K}$ to insure cleanliness of the crystal surface. In addition, when the $\mathrm{H}$ or $\mathrm{D}$ atom hits the surface it has $\sim 7.5 \mathrm{kcal} / \mathrm{mole}$ of translational energy from the beam, chiefly determined by the nozzle temperature. A study of the role of energy transfer in the reaction by varying the $\mathrm{H}$ or $\mathrm{D}$ collision energy would be interesting, but accelerating $\mathrm{H}$ or $\mathrm{D}$ atoms is not a trivial problem. As new sources of $\mathrm{H}$ and $\mathrm{D}$ atoms are developed, perhaps such an investigation will be possible. 


\section{References and Notes}

1. C. Su, Z. G. Dai, W. L. Luo, D. H. Sun, M. F. Vernon, and B. E. Bent, Surf. Sci. 312, 181 (1994); C. Su, H. Q. Hou, G. H. Lee, Z. G. Dai, W.Luo, M. F. Vernon, and B. E. Bent, J. Vac. Sci. Technol. B 11, 1222 (1993); C. Su, M. Xi, Z. G Dai, M. F. Vernon, and B. E. Bent, Surf. Sci. 282, 357 (1993).

2. M. J. Vasile and F. A. Stevie, J. Appl. Phys. 53, 3799 (1982).

3. M. Balooch and D. R. Olander, J. Chem. Phys. 63, 4772 (1975).

4. S. T. Ceyer, D. G. Gladstone, M. McGonigal, and M. T. Schulberg, in Investigations of Surfaces and Interfaces-PartA, edited by B. W. Rossiter and R. C. Baetzold (Wiley, New York, 1993), pp. 383-452.

5. C. A. Becker, J. P. Cowin, L. Wharton, and D. J. Auerbach, J. Chem. Phys. 67, 3394 (1977).

6. S. T. Ceyer, W. L. Guthrie, T. H. Lin, and G. A. Somorjai, J. Chem. Phys. 78, 6982 (1983).

7. D. A. Mantell, K. Kunimori, S. B. Ryali, G. L. Haller, and J. B. Fenn, Surf. Sci. 172, 281 (1986).

8. $\mathrm{D}_{0}^{0}(\mathrm{HF}) \leq 6.40 \mathrm{eV}, \mathrm{D}_{0}^{0}(\mathrm{LiF}) \leq 6.6 \mathrm{eV}$, from Herzberg; G. Herzberg, Molecular Spectra and Molecular Structure, I. Spectra of Diatomic Molecules (D. Van Nostrand Co., Inc., New York, 1950).

9. K. Matsumura, J. Phys. Soc. Jpn. 52, 2164 (1983). 
10. see ref. 9 .

11. Chapter 2 .

12. R. David, K. Kern, P. Zeppenfeld, and G. Comsa, Rev. Sci. Instrum. 57, $2771(1986)$

13. B. Van Zyl and M. W. Gealy, Rev. Sci. Instrum. 57, 359 (1986); H. Koschmieder and V. Raible, Rev. Sci. Instrum. 46, 536 (1975); M. A. D. Fluendy, R. M. Martin, E. E. Muschlitz, Jr., and D. R. Herschbach, J. Chem. Phys. 46, 2172 (1967).

14. The reason the two atomic beams have the same translational energy at the same temperature is that the value of the ratio of the atomic mass to the average mass of the beam is the same. Using $E_{\text {beam }}=7 / 2 \mathrm{kTm} /<\mathrm{m}>$, the maximum energy is calculated, although total rotational cooling (leading to $7 / 2$ instead of $5 / 2$ ) is not expected. Assuming 3\% dissociation versus $1 \%$ dissociation only changes $\mathrm{E}_{\text {beam }}$ by $0.1 \mathrm{kcal} / \mathrm{mole}$. The nozzle temperature is really the determining factor for $\mathrm{E}_{\mathrm{beam}}$.

15. A. M. Wodtke, Ph. D. Thesis, University of California, Berkeley, 1986; X. Zhao, Ph. D. Thesis, University of California, Berkeley, 1988; J. D. Myers, Ph. D. Thesis, University of California, Berkeley, 1993.

16. see ref. 9 .

17. L. D. Weaver and R. H. Hughes, J. Chem. Phys. 52, 2299 (1970).

18. V. Cermak, J. Chem. Phys. 44, 1318 (1966).

19. D. W. Koopman, J. Chem. Phys. 49, 5203 (1968). 
20. H. Hotop, F. W. Lampe, and A. Niehaus, J. Chem. Phys. 51, 593 (1969).

21. see ref. 8 .

22. $\mathrm{E}_{\mathrm{HF}, \mathrm{J}=14}=12.6 \mathrm{kcal} / \mathrm{mole}, \mathrm{E}_{\mathrm{DF}, \mathrm{J}=20}=13.2 \mathrm{kcal} / \mathrm{mole}$; values calculated from the rotational constants, $\mathrm{B}_{\mathrm{HF}}=20.939 \mathrm{~cm}^{-1}$ and $\mathrm{B}_{\mathrm{DF}}=11.007 \mathrm{~cm}^{-1}$ in ref. 8 .

23. M. L. Yu and L. A. DeLouise, Surf. Sci. Rep. 19, 285 (1994).

24. E. W. Kuipers, A. Vardi, A. Danon, and A. Amirav, Phys. Rev. Lett. 66,116 (1991).

25. C. T. Rettner, Phys. Rev. Lett. 69, 383 (1992).

26. see ref. 9. 


\section{Figure Captions}

Fig. 4-1 Nozzle temperature as a function of power. Temperature calculated from neat Ne beam time-of-flight (circles) and measured by optical pyrometry (squares).

Fig. 4-2 Time-of-flight spectra at $\theta_{\mathrm{i}}=30^{\circ}$ comparing the width of the reflected beam with the width of the fast time-of-flight peak due to collisional effects (see text): (a) H-atom beam (solid line) compared to $\mathrm{m} / \mathrm{e}=20$ signal (circles), and (b) D-atom beam (solid line) compared to $\mathrm{m} / \mathrm{e}=21$ signal (circles).

Fig. 4-3 $\mathrm{m} / \mathrm{e}=20$ time-of-flight spectra (circles) showing the second peak fit at all angles, $\theta_{\mathrm{i}}$, by a simulated time-of-flight spectrum (solid line) generated from one translational energy distribution, shown in Fig. 5. The collisional ionization component shown (.....) is not a fit.

Fig. 4-4 $\quad m / e=21$ time-of-flight spectra (circles) showing the second peak fit at all angles, $\theta_{\mathrm{i}}$, by a simulated time-of-flight spectrum (solid line) generated from one translational energy distribution, shown in Fig. 5. The collisional ionization component shown (.....) is not a fit. 
Fig. 4-5 Translational energy distribution used to fit HF and DF data in Figs. 3 and $4 ; \quad<\mathrm{E}>_{\mathrm{T}}=4.3 \mathrm{kcal} / \mathrm{mole}$.

Fig. 4-6 $\quad \theta_{\mathrm{i}}$ distributions for $\mathrm{HF}$ (filled circles) and $\mathrm{DF}$ (open circles) calculated by integrating the simulated time-of-flight spectra for each angle $\theta_{\mathrm{i}}$.

Fig. 4-7 HF translational energy distribution (solid line) compared to a Maxwell-Boltzmann distribution for the surface temperature, $T_{\text {surf }}=690 \mathrm{~K}$ (dashed line). The distributions are normalized.

Fig. 4-8 Microscopic picture of the fluorine end of the diatomic product feeling the "kick" off the surface, justified by HF and DF having the same $E_{\max } \cdot$

Fig. 4-9 Polar plot showing the measured $\theta_{\mathrm{i}}$ distribution for HF (triangles) and a cosine distribution expected if HF were in equilibrium with the surface (squares). For the measured distribution, the normal component of the incident enegy $\left(E_{\perp}\right)$ changes with $\theta_{i}$. For Detection Angle $1 E_{\perp}=2.6$ $\mathrm{kcal} / \mathrm{mole}$, while for Detection Angle $2 \mathrm{E}_{\perp}=6.6 \mathrm{kcal} / \mathrm{mole}$. 


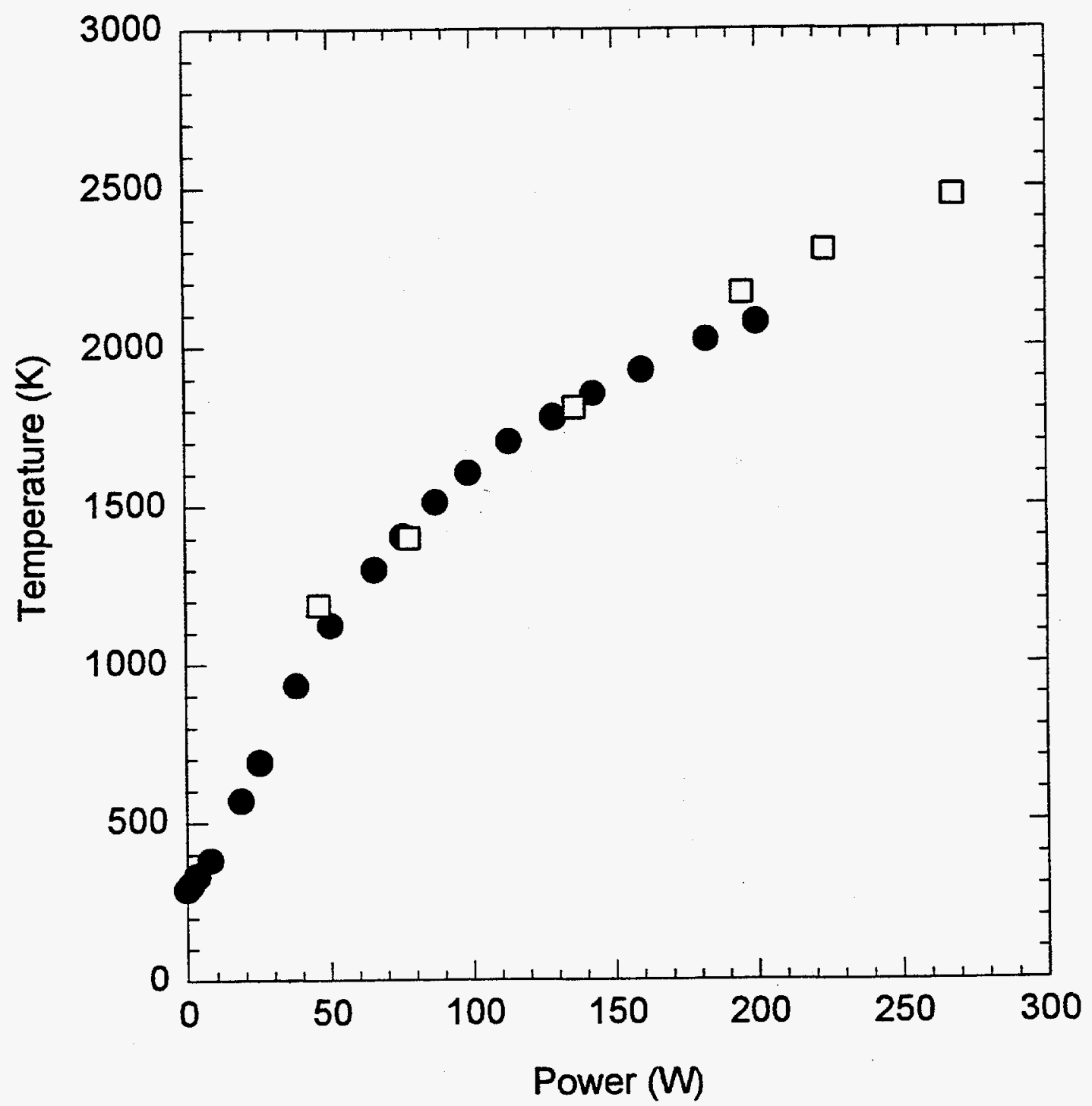

Figure 4-1 


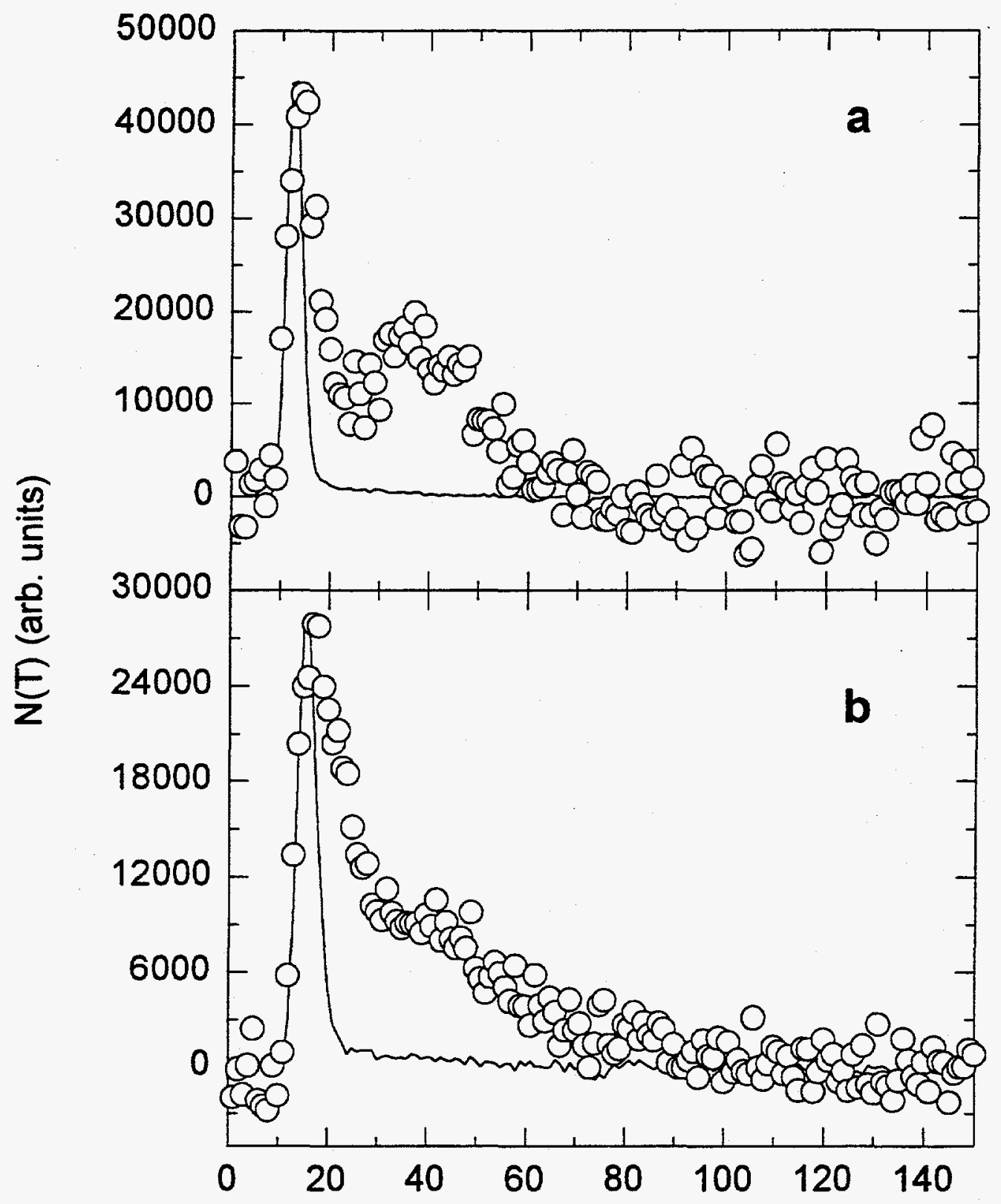

Flight Time, Channels (5.12 $\mu \mathrm{sec} / \mathrm{channel})$

Figure 4-2 


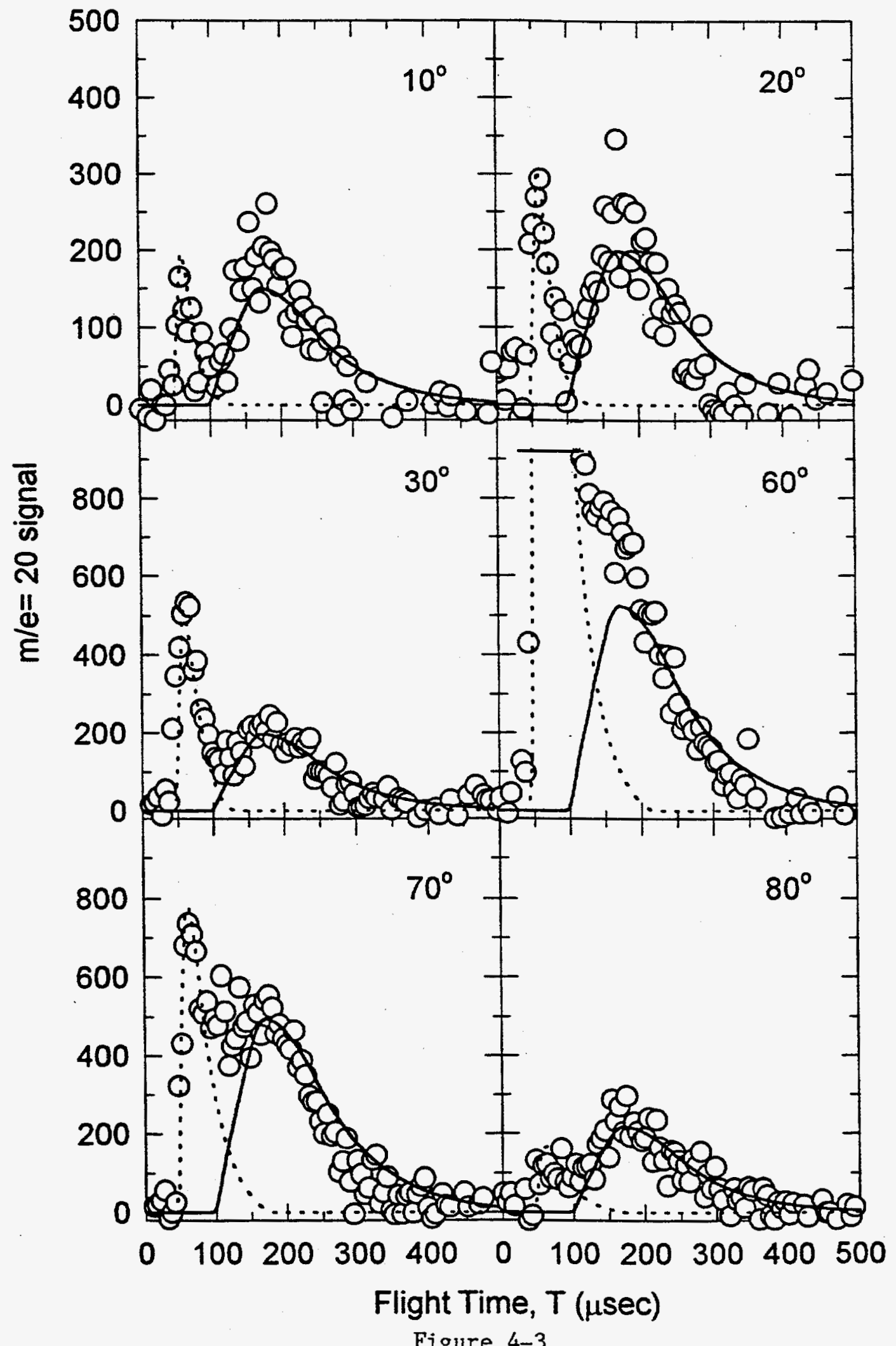

Figure 4-3 


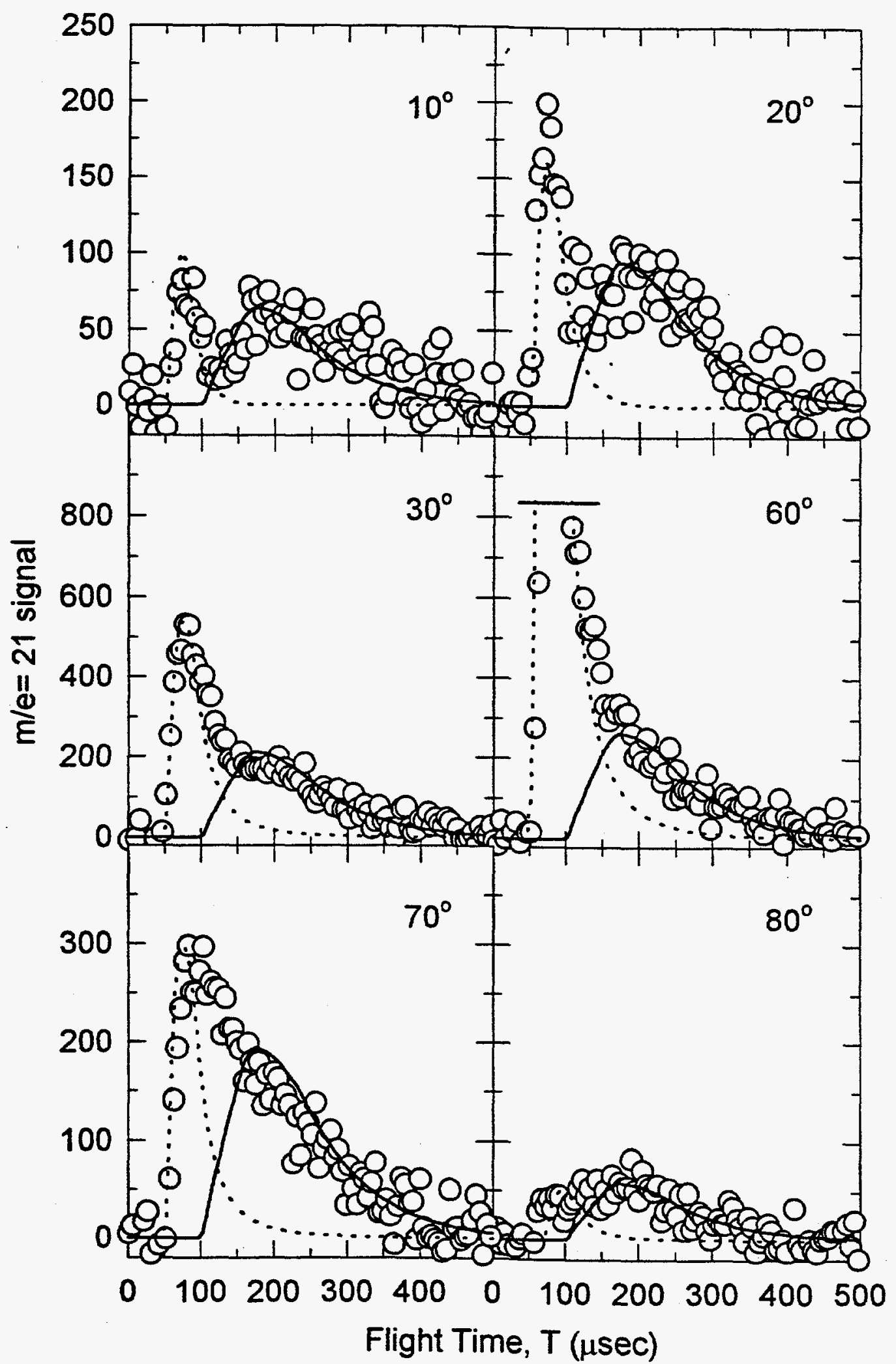

Figure 4-4 


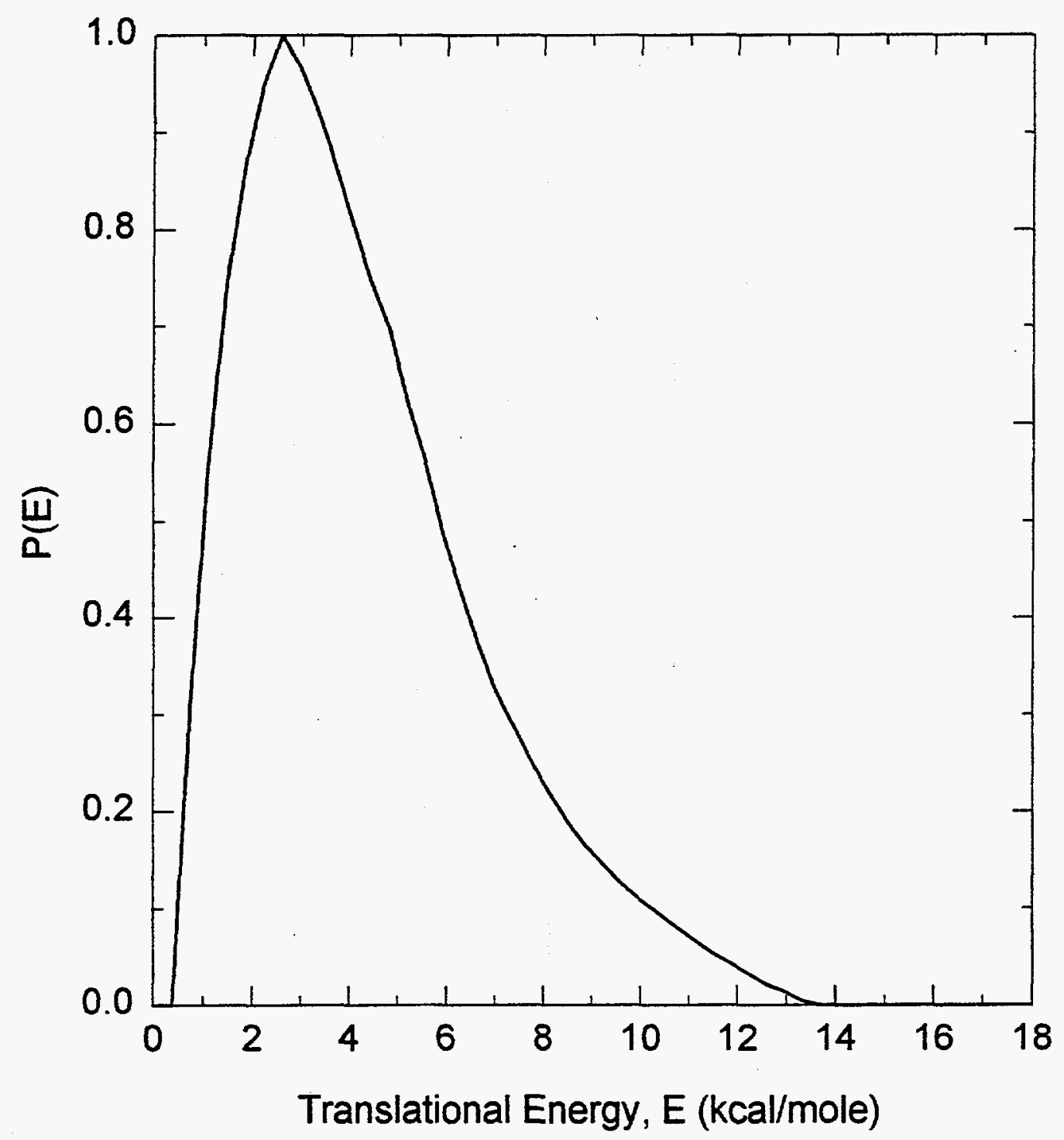

Figure 4-5 


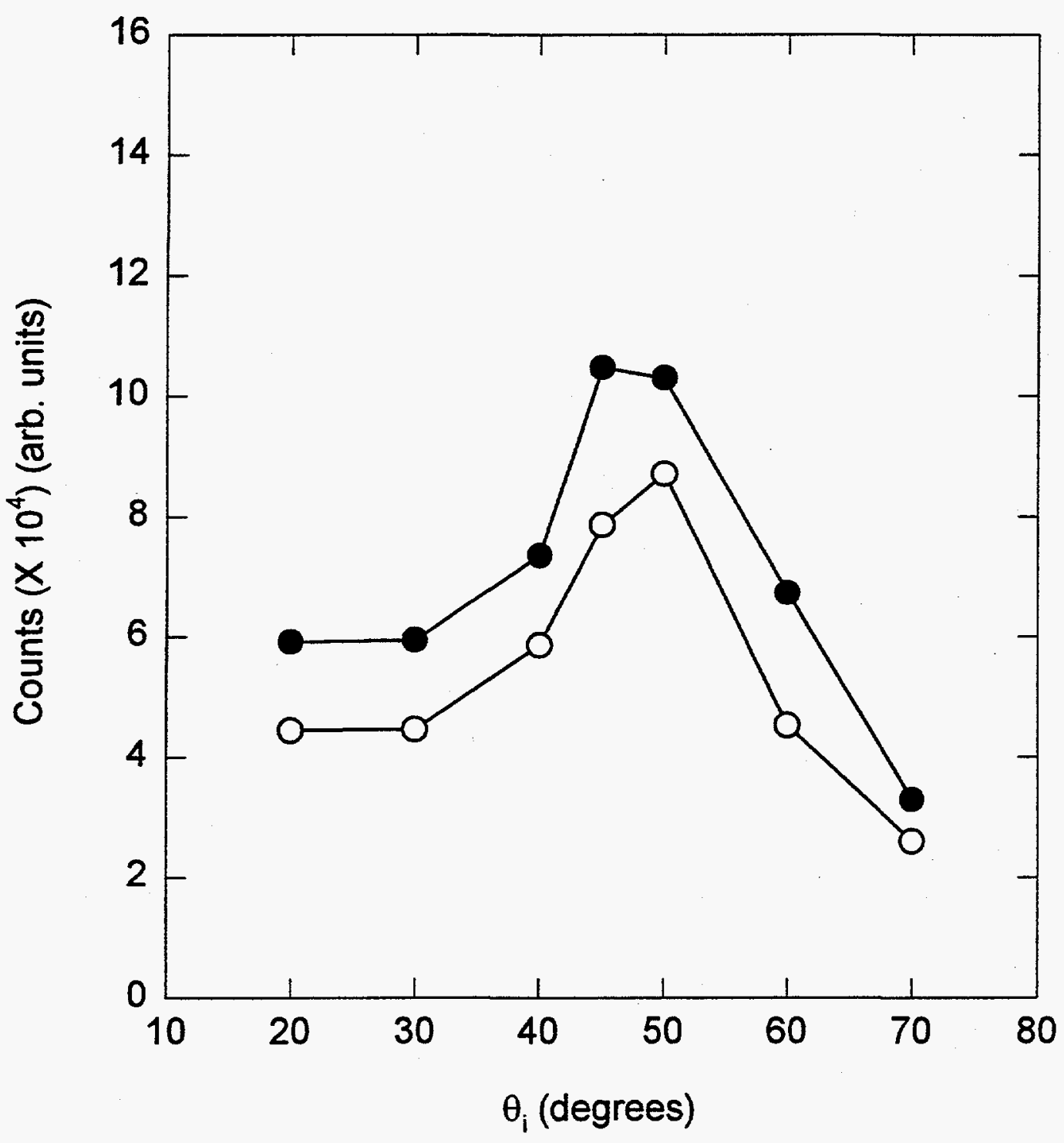

Figure 4-6 


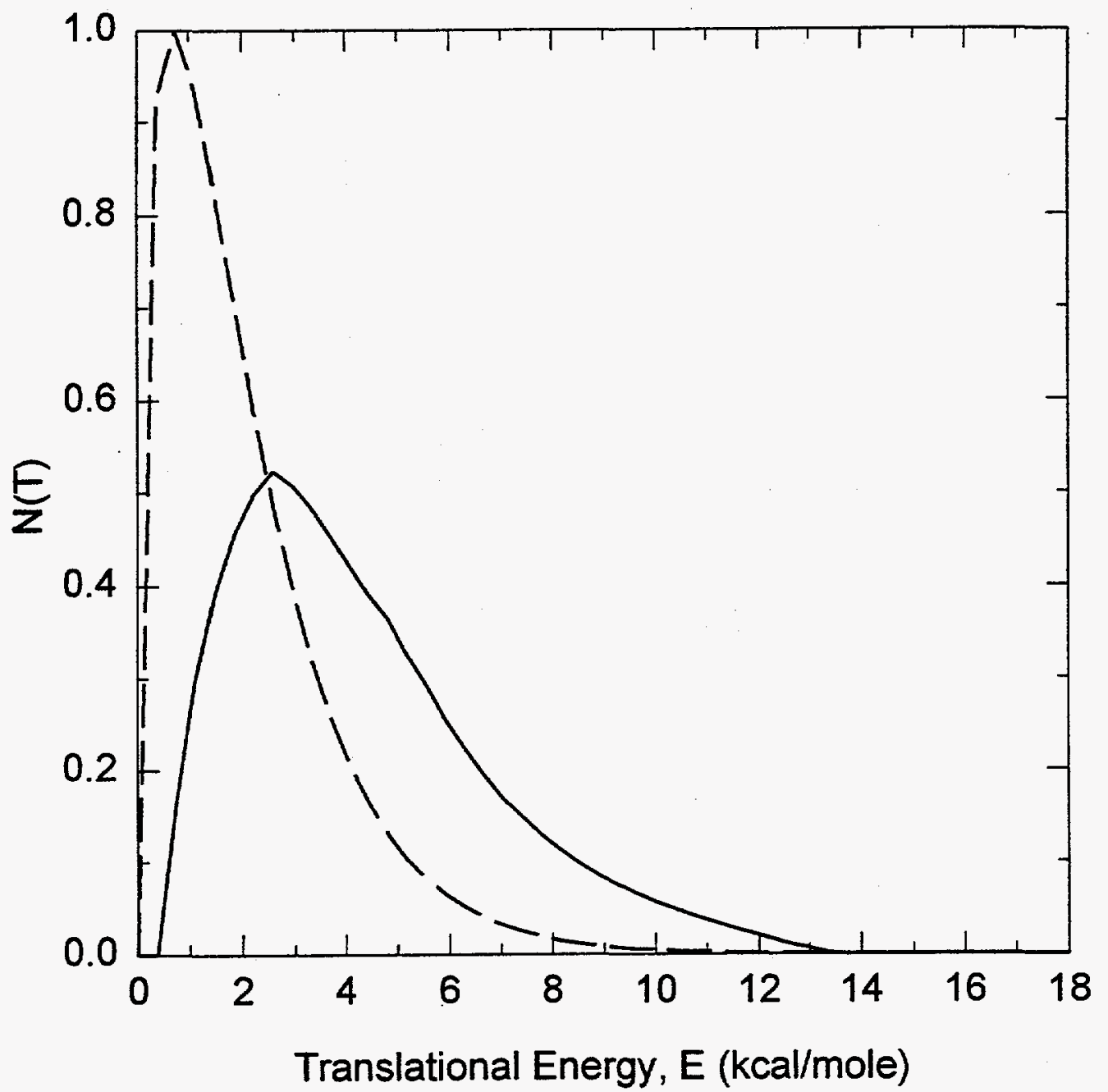

Figure 4-7 


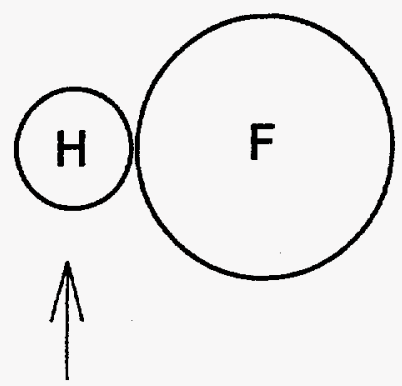

$1 / 2 M_{H} V_{H}^{2}=1 / 2 M_{H F} V_{H F}^{2}$ $\mathrm{V}_{\mathrm{HF}}{ }^{2}=\mathrm{V}_{\mathrm{H}}^{2}\left(\mathrm{M}_{\mathrm{H}} / \mathrm{M}_{\mathrm{HF}}\right)$

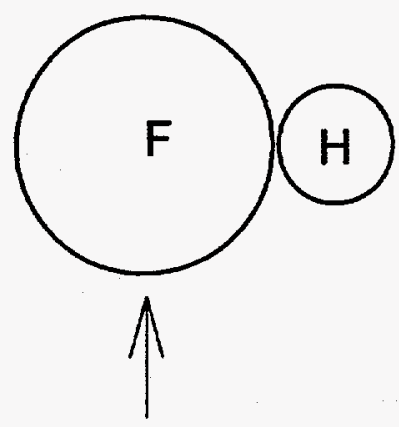

$1 / 2 M_{F} V_{F}^{2}=1 / 2 M_{H F} V_{H F}^{2}$

$$
\mathrm{V}_{\mathrm{HF}}{ }^{2}=\mathrm{V}_{\mathrm{F}}^{2}\left(\mathrm{M}_{\mathrm{F}} / \mathrm{M}_{\mathrm{HF}}\right)
$$

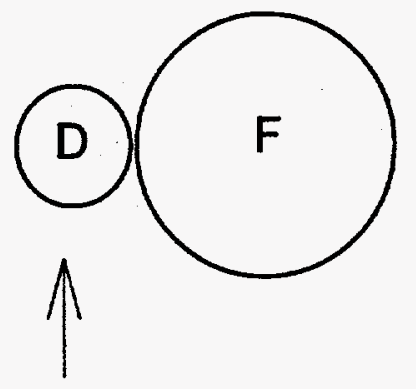

$$
\text { - } \mathrm{V}_{\mathrm{DF}}{ }^{2}=\mathrm{V}_{\mathrm{D}}^{2}\left(\mathrm{M}_{\mathrm{D}} / \mathrm{M}_{\mathrm{DF}}\right)
$$

Expect $4 X$ the Kinetic Energy

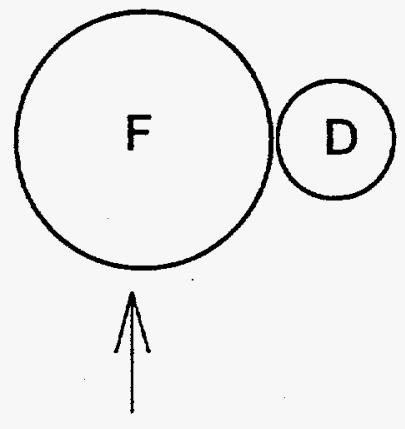

$$
\mathrm{V}_{\mathrm{DF}}{ }^{2}=\mathrm{V}_{\mathrm{F}}{ }^{2}\left(\mathrm{M}_{\mathrm{F}} / \mathrm{M}_{\mathrm{DF}}\right)
$$

Expect the Same Kinetic Energy

Figure 4-8 


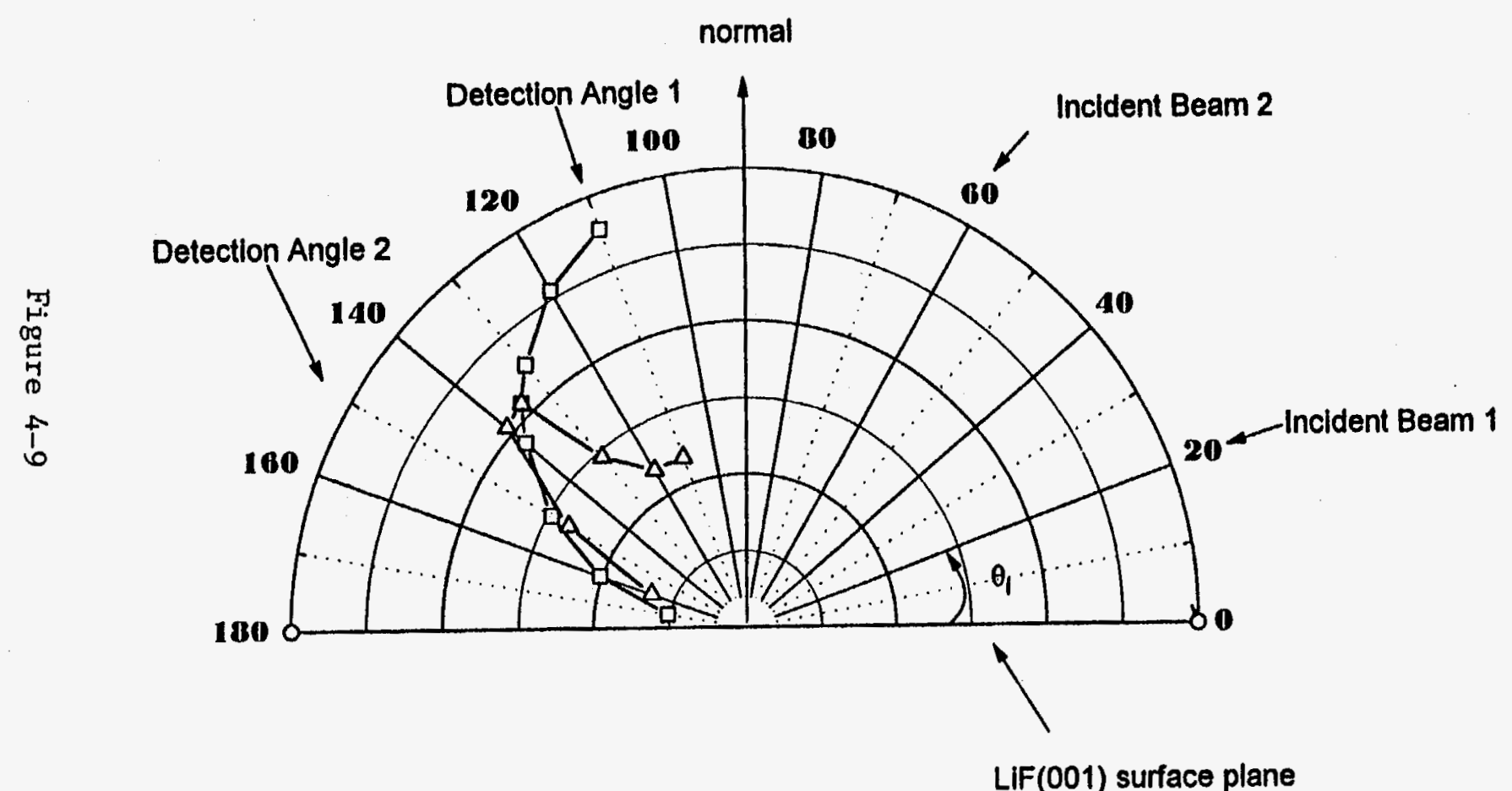




\section{Chapter 5}

\section{Dynamics of the Acetylene- $d_{2}$, Ethylene- $d_{4}$, and Tetradeuteromethane Products from the Reaction of D-Atoms with a Graphite Surface}

\section{Introduction}

Thermodynamic and kinetic aspects of the reaction of hydrogen atoms with graphite have been studied extensively. Early experiments were performed in a bulb by heating the graphite or by passing molecular hydrogen gas over a hot graphite filament or rod to adsorb atomic hydrogen, and then measuring the desorbing gases. The uptake and desorption of $\mathrm{H}_{2}$ was studied ${ }^{1}$ as well as formation and desorption of hydrocarbon species such as $\mathrm{CH}_{4}$ and $\mathrm{C}_{2} \mathrm{H}_{2}{ }^{2}$ Later, the kinetics of the reaction of hydrogen atoms with a graphite surface was studied by dissociating molecular hydrogen in a radiofrequency discharge source. In the surface temperature range of $450-1200 \mathrm{~K}$, the products found were molecular hydrogen and methane. ${ }^{3}$ Using the technique of modulated molecular beams, Balooch et. al. studied the reaction of $\mathrm{H}$-atoms with graphite where the $\mathrm{H}$-atoms were produced in a pyrolytic effusive source. ${ }^{4}$ They found that for surface temperatures up to $800 \mathrm{~K}$, methane was the only product, but acetylene was seen at temperatures above $1000 \mathrm{~K}$. They proposed a model in which the methane was 
formed by sequential addition of adsorbed $\mathrm{H}$-atoms and the acetylene was formed by surface recombination of $\mathrm{CH}$ groups. They did not see any other hydrocarbon products, in particular ethylene, which differs from this work.

While the kinetics of the reacion of $\mathrm{H}$-atoms with graphite have been well studied, the dynamics have not been directly explored. The dynamics in such hydrogen-carbon systems are now of interest, particularly in the related area of diamond growth. In this work, a supersonic beam of $\mathrm{D}$-atoms incident on the basal plane of a graphite surface is used to generate reaction products whose individual velocities are directly measured upon evolution from the surface. By varying the angle, $\theta_{\mathrm{i}}$, between the beam and the surface plane, while the beam-to-detection angle remains fixed at $90^{\circ}$, a distribution of products giving insight into the reaction mechanism is obtained. The dynamics of the deuterated acetylene, ethylene, and methane products are examined. Two reaction mechanisms occur: methane is formed both through the Eley-Rideal and Langmuir-Hinshelwood mechanisms while the acetylene and ethylene are formed through the Langmuir-Hinshelwood mechanism.

\section{Experimental}

A continuous supersonic beam of D-atoms was directed towards the basal plane of highly oriented pyrolitic graphite (HOPG) maintained at $\mathrm{T}_{\text {surf }}=570 \mathrm{~K}$ or $\mathrm{T}_{\text {surf }}=705 \mathrm{~K}$ in a gas-surface scattering apparatus, described previously. ${ }^{5}$ The products of the surface reaction are chopped by a cross-correlation wheel, mass 
selected, and counted by a channeltron after travelling through a flight distance of $23.9 \mathrm{~cm}{ }^{6}$ The time-of-flight spectra of individual mass-selected reaction products were measured for $\mathrm{m} / \mathrm{e}=32,30,28,26,20,18,16$, and 14 . In addition, the reflected beam was monitored.

The surface was mounted on a 3-axis rotatable manipulator equipped with a resistive heater. The HOPG graphite sample was obtained from Union Carbide (grade $\mathrm{ZYH}$ monochromator) and heated for at least 24 hours in vacuum $\left(1 \times 10^{-7}\right.$ torr) to $T_{\text {surf }}=720-770 \mathrm{~K}$ before running an experiment. Surface temperature was limited to a narrow range because of concerns about absorption of background gases and limits of the crystal heater. In the range studied, no surface temperature dependence on the dynamics was clearly seen. Time-of-flight spectra were measured as a function of $\theta_{\mathrm{i}}$, the angle between the source and the surface plane, with the source-to-detector angle fixed at $90^{\circ}$. In such a configuration, any memory of the incident beam in the product dynamics is readily ascertained, but it is not possible to measure a full flux angular distribution to determine energy scaling. In addition, by using a $\mathrm{D}$-atom source, differentiation between deuterium from the beam and hydrogen from the bulk crystal ${ }^{4}$ is possible.

A D-atom source with a flux of $\sim 5 \times 10^{18}$ atoms $\mathrm{cm}^{-2} \mathrm{~s}^{-1}$, previously described, ${ }^{7}$ was used to prepare $D$-atoms by thermal pyrolysis of $D_{2} . D_{2}$ does not react with graphite, ${ }^{4}$ so the atomic species is the true reactant species. In a previous experiment on the $\mathrm{H}$ and $\mathrm{D}$ atom reactions with $\mathrm{LiF}(001)$ to make $\mathrm{HF}$ and DF, respectively, an artifact in the time-of-flight spectra due to collisional 
ionization was identified and discussed extensively. ${ }^{7}$ The same effect is observed in some of the time-of-flight spectra for the D-atom reactions with graphite, and similarly discounted. The collisional ionization arises from the fact that $\mathrm{D}^{+}$, metastable $\mathrm{D}_{2}$, and Rydberg D-atoms are all produced in the electron bombardment ionizer and can collisionally ionize other molecules in the region between the ionizer and the quadrupole filter of the detector. ${ }^{8-11}$ Because the collisional process leads to broadening, which is dependent on $\theta_{i}$ and the particular m/e setting of the quadrupole, there is no systematic way to subtract this artifact from the data. Thus, it is simply excluded from the fitting when analyzing the reaction product data.

\section{Results}

Product time-of-fight spectra were taken for $\mathrm{m} / \mathrm{e}=32,30,28,26,20,18,16$, and 14. Table I lists the assignments of the fragments detected. Signal was not observed above $\mathrm{m} / \mathrm{e}=32$, indicating that $\mathrm{C}_{2}$ is the longest chain hydrocarbon, and no evidence for the evolution of radical species such as $C D, C D_{2}$, or $C_{3}$ was found. ${ }^{4}$ Radical species would be expected to evolve from the surface more slowly than the stable molecular products observed. Additionally, because no time-offlight peak was observed at $\mathrm{m} / \mathrm{e}=36$, ethane- $\mathrm{d}_{6}$ is not a product. The three hydrocarbon products observed were deuterated acetylene, ethylene, and methane. The products will hereafter be referred to by these names with the deuteration understood. The fragmentation patterns for these products and ethane are listed in Table II. ${ }^{12}$ 
By considering the fragmentation patterns, two schemes for fitting the data were devised and tested, one assuming the $C_{2}$ species is ethylene and the other assuming it is ethane. The scheme involving ethane did not fit the data well, ${ }^{13}$ and in addition, since $\mathrm{m} / \mathrm{e}=36$ signal was not observed (which is unique to the ethane product), this possibility can be ruled out. Thus, only one scheme, where the $\mathrm{C}_{2}$ species is ethylene, is discussed here.

The first step of the fitting scheme comes from the fact that $C_{2} D_{4}$ and $C_{2} D_{2}$ do not fragment in the electron bombardment ionizer to $\mathrm{m} / \mathrm{e}=20$. Thus, $\mathrm{m} / \mathrm{e}=20$ data can be fit at all $\theta_{\mathrm{i}}$ to derive a single translational energy distribution describing $\mathrm{CD}_{4}$ evolution. In addition, the fit to the $\mathrm{m} / \mathrm{e}=18$ time-of-flight peak should be generated from the same translational energy distribution since $\mathrm{m} / \mathrm{e}=18$ only appears in the fragmentation pattern of $\mathrm{CD}_{4}$. Fig. 1 shows the time-of-flight data for $\mathrm{m} / \mathrm{e}=20$ and 18 taken at $\theta_{\mathrm{i}}=30^{\circ}$, along with a fit derived from the translational energy distribution in Fig. 2. The fit is a simulated time-of-flight spectrum obtained by the forward convolution method, which includes the various apparatus functions affecting the data. ${ }^{1416}$ Fig. 3 shows how the translational energy distribution of Fig. 2 fits the data at $\theta_{i}=20^{\circ}, 45^{\circ}$, and $60^{\circ}$ for two different surface temperatures, $T_{\text {surf }}=570 \mathrm{~K}$ and $T_{\text {surf }}=705 \mathrm{~K}$. In the $\theta_{\mathrm{i}}=45^{\circ}$ and $60^{\circ}$ data, the fast peak is discounted as an artifact from collisional ionization. ${ }^{7}$ There does not seem to be an appreciable difference in the time-of-flight spectra with surface temperature within the narrow range studied here.

Next, the $m / e=32$ data, attributed solely to $C_{2} D_{4}$, is fit at all angles $\theta_{i}$ to 
generate a single translational energy distribution. This is the distribution describing the dynamics of ethylene evolving from the surface. The fit for $\mathrm{m} / \mathrm{e}=$ 32 at four angles $\theta_{\mathrm{i}}$ is shown in Fig. 4 , and the corresponding translational energy distribution is in Fig. 5. The $m / e=26$ data has two contributions, one from $C_{2} D_{4}$, and the other from $C_{2} D_{2}$. Once the $C_{2} D_{4}$ distribution has been determined from the $\mathrm{m} / \mathrm{e}=32$ data, the translational energy distribution of $\mathrm{C}_{2} \mathrm{D}_{2}$ is adjusted to generate the best total fit to the $\mathrm{m} / \mathrm{e}=26$ data at all angles $\theta_{i}$, while also finding the relative contributions of the two channels. The fit for $\theta_{i}=20^{\circ}, 45^{\circ}$, and $60^{\circ}$ is shown for two surface temperatures in Fig. 6 . Once again, the time-of-flight data do not show a dependence on $T_{\text {surf }}$ in the range studied. The acetylene translational energy distribution used to fit the $\mathrm{m} / \mathrm{e}=26$ data is shown in Fig. 7 .

The time-of-flight spectra at $\mathrm{m} / \mathrm{e}=16$ and 14 should be fit with a combination of the translational energy distributions already derived for methane, ethylene, and acetylene. In the case of $\mathrm{m} / \mathrm{e}=16$, the data can be fit using a combination of the distributions for ethylene and methane; acetylene only gives a small amount of $\mathrm{m} / \mathrm{e}=16$ upon fragmentation in the ionizer. The $\mathrm{m} / \mathrm{e}=16$ data is shown in Fig. 8 with a fit generated from a combination of the translational energy distributions for methane, Fig. 3, and ethylene, Fig. 5. Data for $\mathrm{m} / \mathrm{e}=14$ is fit using a combination of all three translational energy distributions found above, Fig. 9. The contribution from methane is negligible, which is at first surprising when only considering the fragmentation pattern of the different products. It turns out that methane is the minor product of the gas-surface reaction under the conditions 
used, though, as will be discussed later. Therefore, it is reasonable that the methane contribution is negligible compared to acetylene and ethylene in the $\mathrm{m} / \mathrm{e}=$ 14 time-of-flight spectrum. Finally, data at $\mathrm{m} / \mathrm{e}=30$ must be a result of ethylene fragmenting in the electron bombardment ionizer. In Fig. 10, time-of-flight data taken for $\mathrm{m} / \mathrm{e}=30$ is shown $\theta_{\mathrm{i}}=20^{\circ}$ with a fit generated from the ethylene translational energy distribution in Fig. 5.

In Fig. 11, the three translational energy distributions used to fit the data are plotted with a Maxwell-Boltzmann distribution for $\mathrm{T}_{\text {surf }}=705 \mathrm{~K}$. The distributions for the products clearly peak at higher energies than the Maxwell-Boltzmann distribution, indicating that there is a barrier to desorption of the product molecules. For the product translational energy distributions, $\left\langle\mathrm{E}>_{\mathrm{T}}=3.1 \mathrm{kcal} / \mathrm{mole}\right.$ for ethylene, $<\mathrm{E}>_{\mathrm{T}}=4.1 \mathrm{kcal} / \mathrm{mole}$ for acetylene, and $\left\langle\mathrm{E}>_{\mathrm{T}}=3.9 \mathrm{kcal} / \mathrm{mole}\right.$ for methane. The $<\mathrm{E}>_{\mathrm{T}}$ for acetylene and methane is nearly the same, and the shapes of the distributions are similar, but the low-energy side of the acetylene distribution is shifted to higher energy as is the peak, by about $0.5 \mathrm{kcal} / \mathrm{mole}$. It turns out that the methane distribution is reflecting the translational energy release from two different surface reaction mechanisms, which will be addressed in a discussion of the $\theta_{i}$ product distributions. Since the mechanism of acetylene and methane formation is different, as evidenced by the $\theta_{\mathrm{i}}$ distributions (see below), the similarity of the two translational energy distributions is thought to be just coincidental. Ethylene has $E_{\max } \sim 9 \mathrm{kcal} / \mathrm{mole}$, which is lower than the common $E_{\max } \sim 11 \mathrm{kcal} / \mathrm{mole}$ for acetylene and methane. ${ }^{17}$ 
Qualitatively, the yield of the products under the conditions used indicates that the major product is ethylene and the minor product is methane. However, the relative yield of products is expected to be dependent on the D-atom flux and so no attempt to quantify a branching ratio is made here. The flux of D-atoms used in this experiment is about 100 times higher than in the modulated molecular beam work of Balooch et. al. (where ethylene product was not observed), ${ }^{4}$ suggesting that ethylene formation is dependent on the availability of D-atoms.

The $\theta_{i}$ distributions for each product were measured, and the results are plotted in Figs. $12-14$ in polar form along with a $\cos (\theta)$ distribution, which is scaled to the product intensity for $\theta_{\mathrm{i}}=45^{\circ}$. For the $\theta_{\mathrm{i}}$ distribution, it is important to remember that the beam-to-detector angle remains fixed at $90^{\circ}$, and thus the normal component of the $\mathrm{D}$-atom incident energy changes with $\theta_{\mathrm{i}}$. The $\cos (\theta)$ distribution shown here would be observed if the product: 1) was in thermal equilibrium with the surface at the surface temperature and 2) had no "memory" of the incident beam. In Figs. 12-14 the beam is incident on the surface between $\theta_{i}=0-90^{\circ}$, and the products are detected from $\theta=90-180^{\circ}$ as shown. The angle $\theta_{i}$ is thus equal to the angle between the detector and the surface normal for each measurement. The acetylene $\theta_{\mathrm{i}}$ distribution (Fig. 12) is most similar to a $\cos (\theta)$ distribution. The ethylene $\theta_{\mathrm{i}}$ distribution is somewhat similar to a $\cos (\theta)$ distribution, except the product intensity is more strongly peaked toward the surface normal (Fig. 13). Methane, however, differs from the acetylene and ethylene; part of its distribution is strongly peaked toward $45^{\circ}$ and part looks like a $\cos ^{\mathrm{n}}(\theta)$ distribution (Fig. 14). 
The $\theta_{\mathrm{i}}$ distributions show that some of the methane product has a "memory" of the incident beam, while all of the acetylene and ethylene products do not. The important dynamical implications of this result will be discussed in the following section.

\section{Discussion}

\section{Product Translational Energy}

The translational energy and $\theta_{i}$ distributions of the deuterated methane, acetylene, and ethylene products reveal some dynamical and mechanistic aspects of the gas-surface reaction. The importance of considering both the energy released into translation and the angular distribution of products has been demonstrated. ${ }^{18}$ Measuring an angular distribution gives insight into the reaction mechanism and desorption dynamics, but can be misleading without an accompanying translational energy distribution. This is particularly true when $\operatorname{a} \cos (\theta)$ angular distribution is observed, which is normally associated with thermal desorption but can also arise simply from the way a molecule's energy is accommodated at the surface before desorption (i.e. partitioned between the directions parallel and perpendicular to the surface).

The translational energy distributions in Fig. 11 have been normalized to have the same area. All three peak at higher energies than a Maxwell-Boltzmann distribution for $\mathrm{T}_{\text {surf }}=705 \mathrm{~K}$ (shown) and of course for $\mathrm{T}_{\text {surf }}=570 \mathrm{~K}$ (not shown). This is indicative of a barrier in the exit channel of each potential energy surface 
governing the interaction of individual products with the graphite surface. ${ }^{19}$ If the exit channels had no barrier, Maxwell-Boltzmann distributions would be expected. Deviations from Maxwell-Boltzmann behavior characteristic of $\mathrm{T}_{\text {surace }}$ have been observed in many studies of desorption. ${ }^{20-22}$ In fact, Comsa has pointed out that there is really no general reason to expect to see Maxwell-Boltzmann distributions for the desorption of molecules from a surface. ${ }^{23}$ The distributions can be loosely characterized by a "temperature" $\left(\mathrm{T}_{<\mathrm{B}}=\left\langle\mathrm{E}>_{\mathrm{T}} / 2 \mathrm{k}_{\mathrm{B}}\right.\right.$, where $\mathrm{k}_{\mathrm{B}}$ is the Boltzmann constant) to quantify the deviation from $\mathrm{T}_{\text {surface}} ; \mathrm{T}_{\angle B}=\mathrm{T}_{\text {surface }}$ in the case of a Maxwell-Boltzmann distribution. ${ }^{24}$ For ethylene, acetylene, and methane, $T_{\triangle \mathrm{B}}=$ 780,1032 , and $982 \mathrm{~K}$, respectively.

The three product distributions are also wider than a Maxwell-Boltzmann distribtution. As the newly formed product surmounts the barrier and leaves the surface, potential energy is not only channelled into translation but also into internal modes of the product, which is reflected in the width of the derived translational energy distribution. Products with less translational energy are more internally excited. The width can also reflect reaction at different types of surface sites, which will effectively change the total available energy to the departing molecule. For the two products that desorb without a memory of the incident beam, the width is larger for ethylene, which has more degrees of freedom than acetylene. The methane is formed through two mechanisms, a direct reaction and a surface recombination, and the combination of the two mechanisms or reaction from different types of surface sites may broaden the distribution. 
The ethylene distribution has the lowest $\mathrm{T}_{<B}(780 \mathrm{~K})$ and also has a lower $\mathrm{E}_{\max }$ of $\sim 9 \mathrm{kcal} / \mathrm{mole}$. Such behavior is consistent with a lower barrier height with respect to the final product state in the exit channel for the ethylene product as compared to the acetylene or methane. The fact that ethylene was found to be the major product under the conditions used also suggests a lower barrier in the forward direction, from the reactants to the transition state. The ratio of products ethylene: acetylene: methane is $\sim 9: 3: 1$. The barrier in the exit channel can result from the geometric and configurational requirements of the transition state for a particular product to form and evolve. In the case of ethylene, the carbon has the same $\mathrm{sp}^{2}$ hybridization of the graphitic carbon on the surface. Perhaps this explains why ethylene evolution would have a lower barrier.

\section{Product $\theta_{\mathrm{i}}$ Distributions}

The $\theta_{\mathrm{i}}$ distributions in Figs. 12-14 illustrate how the product flux changes when the surface is rotated. In the rotating surface, fixed source-detector arrangement, the normal component of the incident $D$-atom energy varies with $\theta_{i}$. Thus, the distribution observed cannot be properly fit to $a \cos ^{\mathrm{n}}(\theta)$ distribution unless there is no effect from the incident beam. However, in the case of acetylene the distribution clearly shows a loss of "memory" of the incident beam. It is interesting to note, in fact, that the angle with the most product intensity here is from the measurement where the D-atom has the least incident energy in the normal direction. A mechanistic picture whereby two $\mathrm{CD}$ groups move around on 
the surface, find each other, and join together to evolve as acetylene is consistent with no "memory" of the beam, which is the Langmuir-Hinshelwood type mechanism. ${ }^{25}$ The plotted $\cos (\theta)$ distribution, shown for comparison, is what would be seen if a true thermal desorption were occurring with the product in equilibrium with the surface. However, since the translational energy distribution clearly peaks at higher energy than a Maxwell-Boltzmann distribution, thermal desorption is not an appropriate description of the desorption dynamics. The observed $\theta_{\mathrm{i}}$ distribution must arise from the dynamics of the last formation step and exit off the surface, reflecting the shape of the exit barrier in the potential energy surface. While the CD groups are accomodated to the surface, the newly formed acetylene is not, for there is no surface temperature dependence observed. The acetylene product formation and desorption can be a concerted process, resulting in a very short residence time for the acetylene on the surface. Thus the $\cos (\theta)$ distribution reflects the way the acetylene's energy is divided in the directions parallel and perpendicular to the surface as it moves through the exit channel of the potential energy surface.

The $\theta_{i}$ distribution for ethylene, Fig. 13, also shows a loss of "memory" of the beam. The distribution is more highly peaked toward the normal than a $\cos (\theta)$ distribution, as shown. In fact, it can be fit to a $\cos ^{2.6}(\theta)$ distribution, again based on loss of "memory" of the incident beam. The translational energy distribution already indicates that the ethylene evolution is not a simple thermal desorption. A microscopic picture of two accomodated $\mathrm{CD}_{2}$ groups diffusing along the surface 
until they find each other and form the product is consistent with the angular distribution, which is again the Langmuir-Hinshelwood mechanism. However, the strong peaking indicates that the exit channel barrier has a different shape than that in the acetylene case. This barrier is more significant along the normal direction. The ethylene product, whose dynamics show no surface temperature dependence, is not accomodated to the surface. In addition, its energy must be partitioned more in the direction perpendicular to the surface than parallel to the surface in order to result in the $\cos ^{2.6}(\theta)$ distribution. Peaked angular distributions have been previously interpreted as resulting from excess energy in the desorbing molecule in a study of $\mathrm{CO}_{2}$ formed by reaction of $\mathrm{CO}$ and oxygen $\left(\mathrm{O}_{2}{ }^{2-}\right.$ and $\left.\mathrm{O}\right)$ on a $\mathrm{Pt}(111)$ surface. ${ }^{26}$ There may be a particular configuration of the transition state that leads to more energy in the coordinate normal to the surface.

Fig. 14 shows the $\theta_{\mathrm{i}}$ distribution for the methane product. Methane differs from acetylene and ethylene: it appears that two mechanisms, Eley-Rideal and Langmuir-Hinshelwood, are operating simultaneously. One part of the distribution is highly peaked toward $45^{\circ}$, indicating that there is a "memory" of the incident beam. This part of the angular picture is consistent with $\mathrm{D}$-atom addition from the beam to radical species adsorbed on the surface: first to $C D_{(a d s)}$, then to $C D_{2(\text { ads) }}$, and finally to $\mathrm{CD}_{3(\mathrm{ads})}$, constituting an Eley-Rideal mechanism. Sequential additions from adsorbed D-atoms or D-atoms that have suffered a few collisions with the surface before reaction (and therefor lost information about their incident coordinates) contribute to the other part of the angular distribution, which has a 
$\cos ^{3.4}(\theta)$ distribution (note that this fit is only based on three points, so it is rough). The $\cos ^{3.4}(\theta)$ contribution is from reactions of the Langmuir-Hinshelwood type mechanism. Thus, both the Eley-Rideal and Langmuir-Hinshelwood mechanisms are occuring simultaneously. Unfortunately, it is not possible to deconvolute the translational energy distribution (Fig. 2) to reflect the individual contributions from the two mechanisms. Reports of the Eley-Rideal mechanism are quite rare, and so the observation of such a mechanism in this case is of particular interest. ${ }^{27-29}$ From kinetic studies, ${ }^{4}$ the addition to $\mathrm{CD}_{2}$ is expected to be the rate-determining step while the addition to $\mathrm{CD}_{3}$ is very fast. Thus, if there is a competition between $\mathrm{D}$ atom addition to $C D_{3}$ and two $\mathrm{CD}_{3}$ groups finding each other on the surface to form ethane, the D-atom addition would be favored. Perhaps this is why no evidence for ethane formation has been observed.

\section{Microscopic Picture}

In the present study, the observed products were methane, acetylene, and ethylene. While methane and acetylene were reported previously in a modulated molecular beam study, ${ }^{4}$ ethylene was not. Possible explanations for this discrepancy are that the flux of D-atoms is two orders of magnitude higher here, and the $\mathrm{D}$-atoms are produced in a supersonic expansion with $\mathrm{E}_{\text {incident }} \sim 7.5$ $\mathrm{kcal} / \mathrm{mole}$. Previously, acetylene formation was only seen at high surface temperatures (above $1000 \mathrm{~K}$ ), and methane was the only hydrocarbon product at lower temperatures (up to $800 \mathrm{~K}$ ). This suggests that the surface temperature had 
to be high enough to insure mobility of the $\mathrm{CD}_{(\text {ads }}$ species on the surface so that they could find each other with a reasonable frequency. The incident beam energy of $\sim 7.5 \mathrm{kcal} / \mathrm{mole}$ is well above $\mathrm{k}_{\mathrm{B}} \mathrm{T}_{\text {surf }}$ at $1000 \mathrm{~K}\left(\mathrm{k}_{\mathrm{B}} \mathrm{T}_{\text {surf }}=1.99 \mathrm{kcal} / \mathrm{mole}\right)$, so there may be ample energy available for mobility of newly formed species, depending on how much energy is consumed in the initial reaction. Thus, the incident energy from the D-atom can be partially accommodated by $\mathrm{CD}$ and $\mathrm{CD}_{2}$ mobility at the surface, which is necessary for recombination reactions to form acetylene and ethylene.

In further considering the mobility of the radical species at the surface, it becomes clear why ethane formation is not favored. The graphitic carbon atom starts as an $\mathrm{sp}^{2}$ carbon, covalently bound to three other carbon atoms. As D-atom addition occurs, one $\mathrm{C}-\mathrm{C}$ bond is broken and a D-atom is attached. However, a CD species is still anchored to the surface by two other C-C bonds if the $\mathrm{sp}^{2}$ hybridization is maintained. Likewise, a $\mathrm{CD}_{2}$ species is anchored by one $\mathrm{C}-\mathrm{C}$ bond. $\mathrm{A} \mathrm{CD}_{3}$ species loses its anchor, though, if $\mathrm{sp}^{2}$ hybridization is maintained, and thus may not remain on the surface long enough to recombine with another $\mathrm{CD}_{3}$ group. The final $\mathrm{D}$-atom addition quickly forms $\mathrm{CD}_{4}$, and thus $\mathrm{CD}_{3}$ may be a very shortlived species under conditions of high D-atom flux. This would explain the absence of ethane product. In addition, radical species evolution would probably be unfavorable. 


\section{Comparison with other Molecular Beam Etching Studies}

The dynamics of only a few etching reactions have been studied using molecular beam-surface scattering. Some are mentioned here for the sake of comparison in order to gain a better understanding of the dynamics observed in the D-atom/graphite system. Of particular interest is why some systems show barrierless thermal desorption behavior and others do not.

The dynamics of $\mathrm{Cl}_{2}$ etching of $\mathrm{GaAs}$ have been studied. In one experiment, the etching of the $\mathrm{GaAs}(110)$ surface to form $\mathrm{GaCl}_{3}$ was reported, ${ }^{30}$ with $\mathrm{GaCl}_{3}$ evolution well described by a Maxwell-Boltzmann distribution with a temperature below $\mathrm{T}_{\text {surface }}$ and a cosine angular distribution. These results were attributed to an absence of an activation barrier for desorption. In another experiment, ${ }^{31}$ time-of-flight spectra of the $\mathrm{GaCl}$ from $\mathrm{Cl}_{2}$ reacting with $\mathrm{GaAs}(100)$ were measured. A cosine distribution for $\mathrm{GaCl}$ was observed, and the authors mention that an even better fit was found for $\cos ^{1.5}(\theta)$, i.e. more peaked towards the surface normal than $\cos (\theta)$. The time-of-flight spectrum was wider than a Maxwell-Boltzmann distribution (the width was dependent on $T_{\text {suface }}$ with slower products appearing at lower surface temperatures) except at a high surface temperature of $550{ }^{\circ} \mathrm{C}$. Attempts to fit the time-of-flight distribution for $\mathrm{GaCl}$ by including a factor accounting for a surface residence time were unsuccessful. It was postulated, however, that $\mathrm{GaCl}$ comes from different types of defect sites, giving rise to different residence times and thus broadens the time-of-flight distribution. 
The velocities of $\operatorname{SiCl}_{n}(n=2,3$ and 4) reaction products from the reaction of $\mathrm{Cl}_{2}$ with $\mathrm{Si}(111)$ were measured. ${ }^{19}$ The product time-of-flight distributions were well fit with Maxwell-Boltzmann distributions with temperatures $9.0 \%$ higher than $\mathrm{T}_{\text {surface }}$. In addition, the angular distribution was a bit peaked toward the normal, fit by $\cos ^{1.26}(\theta)$. The $\mathrm{SiCl}_{2}$ results were explained in terms of a low potential barrier in the exit channel resulting in translational heating of the newly formed products upon desorption.

The results presented here show broader product distributions than expected for a Maxwell-Boltzmann distribution as in the $\mathrm{GaCl}$ work, which could arise for similar reasons, i.e. reaction and desorption from different types of surface sites. In addition, the ethylene $\theta_{\mathrm{i}}$ distribution is more highly peaked towards the normal than a $\cos (\theta)$ distribution as observed for the $\mathrm{SiCl}_{2}$ and also the $\mathrm{GaCl}$. The acetylene distribution follows $\cos (\theta)$ well, but the translational energy distribution shows that thermal desorption is not taking place. As in the case of $\mathrm{SiCl}_{2}$ formation and desorption, there is a barrier in the exit channel for each product, indicated by the translational energy distribution peaking at higher energy than a MaxwellBoltzmann distribution for the surface temperature.

\section{Conclusion}

The dynamics of three hydrocarbon reaction products in the reaction of $\mathrm{D}$ atoms with a graphite surface have been explored. Time-of-flight measurements of individual products coupled with variation of the beam-surface angle allow for 
determination of the translational energy imparted to each product and the role of the incident beam in each product's formation on the surface. Unlike some other etching reactions reported in the literature, the products do not evolve by a simple thermal desorption process. In fact, there is a barrier in the exit channel for each product, and thus the translational energy distribution is shifted to higher energies than a Maxwell-Boltzmann distribution for the surface temperature. Additionally, the distributions do not have the functional form of a Maxwell-Boltzmann distribution. By examining the influence of the incident $\mathrm{D}$-atom beam, it was determined that the methane was formed both by sequential addition of D-atoms from the beam to radical species on the surface (Eley-Rideal mechanism) and also through a Langmuir-Hinshelwood type mechanism, while acetylene and ethylene were formed by recombination of accommodated $\mathrm{CD}$ and $\mathrm{CD}_{2}$ species on the surface, respectively (Langmuir-Hinshelwood). The accommodation of the incident energy allows for surface mobility and the radical species lose "memory" of the incident D-atom velocity. The major product was ethylene under the conditions of D-atom flux and incident energy used. A study of the branching ratio of products by varying the D-atom flux would be interesting and would allow for a greater understanding of the competition between the pathways leading to the three observed products. 


\section{References and Notes}

1. J. P. Redmond and P. L. Walker, Jr., J. Phys. Chem. 54, 1093 (1960).

2. J. T. Clarke and B. R. Fox, J. Chem. Phys. 46, 827 (1967).

3. B. J. Wood and H. Wise, J. Phys. Chem. 73, 1348 (1969).

4. M. Balooch and D. R. Olander, J. Chem. Phys. 63, 4772 (1975).

5. see Chapter 2.

6. R. David, K. Kern, P. Zeppenfeld, and G. Comsa, Rev. Sci. Instrum. 57, 2771 (1986).

7. see Chapter 4.

8. L. D. Weaver and R. H. Hughes, J. Chem. Phys. 52, 2299 (1970).

9. V. Cermak, J. Chem. Phys. 44, 1318 (1966).

10. D. W. Koopman, J. Chem. Phys. 49, 5203 (1968).

11. H. Hotop, F. W. Lampe, and A. Niehaus, J. Chem. Phys. 51, 593 (1969).

12. E. Stenhagen, S. Abrahamsson, and F. W. McLafferty, Atlas of Mass

Spectral Data (John Wiley and Sons, New York, 1969).

13. A fitting scheme assuming that $\mathrm{m} / \mathrm{e}=32$ was due to ethane did not fit the data well. In particular, one consistent translational energy distribution could not be found to fit the $\mathrm{m} / \mathrm{e}=26$ data.

14. A. M. Wodtke, Ph. D. Thesis, University of California, Berkeley, 1986.

15. X. Zhao, Ph. D. Thesis, University of California, Berkeley, 1988. 
16. CMLAB2 fitting program written by J. D. Myers.

17. The uncertainty at the tail end of the translational energy distribution from the $5 \mu \mathrm{sec}$ resolution of the chopper (double sequence cross-correlation wheel) is $\sim 1 \mathrm{kcal} / \mathrm{mole}$.

18. S. T. Ceyer, W. L. Gutherie, T. H. Lin, and G. A. Somorjai, J. Chem. Phys. 78, 6982 (1983).

19. H. Yoshikawa and K. Shobatake, Chem. Phys. Lett., 223, 341 (1994).

20. C. T. Rettner, E. K. Schweizer, and C. B. Mullins, J. Chem. Phys. 90, 3800 (1989).

21. C. R. Arumainayagam, R. J. Madix, M. C. McMaster, V. M. Suzawa, and J. C. Tully, Surf. Sci. 226, 180 (1990).

22. L. A. DeLouise, J. Chem. Phys. 94, 1528 (1991).

23. G. Comsa and R. David, Surf. Sci. Rep. 5, 145 (1985).

24. While $\mathrm{T}_{<\mathrm{E}>}=<\mathrm{E}>_{\mathrm{T}} / 2 \mathrm{k}_{\mathrm{B}}$ is only strictly valid for a Maxwell-Boltzmann distribution, it is often used loosely to quantify deviations in the data from a Maxwell-Boltzmann distribution. See Ref. 23.

25. J. I. Steinfeld, J. S. Francisco, and W. L. Hase, Chemical Kinetics and Dynamics (Prentice Hall International, Inc., London, 1989) p.196.

26. T. Matsushima, Surf. Sci., 123, L663 (1982).

27. M. L. Yu and L. A. DeLouise, Surf. Sci. Rep. 19, 285 (1994).

28. E. W. Kuipers, A. Vardi, A. Danon, and A. Amirav, Phys. Rev. Lett. 66, $116(1991)$. 
29. C. T. Rettner, Phys. Rev. Lett. 69, 383 (1992).

30. L. A. DeLouise, J. Chem. Phys. 94, 1528 (1991).

31. P. Bond, P. N. Brier, J. Fletcher, P. A. Gorry, and M. E. Pemble, Chem. Phys. Lett., 208, 269 (1993). 
Table I. Assignment of Detected

Ions from Reaction

\begin{tabular}{|c|c|}
\hline $\mathrm{m} / \mathrm{e}$ & $\begin{array}{l}\text { parent ion } \\
\text { assignment }\end{array}$ \\
\hline 32 & $\mathrm{C}_{2} \mathrm{D}_{4}^{+}$ \\
\hline 30 & $\mathrm{C}_{2} \mathrm{D}_{4}^{+}$ \\
\hline 28 & $\begin{array}{l}\mathrm{C}_{2} \mathrm{D}_{4}^{+} \\
\mathrm{C}_{2} \mathrm{D}_{2}^{+}\end{array}$ \\
\hline 26 & $\begin{array}{l}\mathrm{C}_{2} \mathrm{D}_{2}^{+} \\
\mathrm{C}_{2} \mathrm{D}_{4}^{+}\end{array}$ \\
\hline 20 & $\mathrm{CD}_{4}^{+}$ \\
\hline 18 & $\mathrm{CD}_{4}^{+}$ \\
\hline 16 & $\begin{array}{l}\mathrm{CD}_{4}^{+} \\
\mathrm{C}_{2} \mathrm{D}_{4}^{+} \\
\mathrm{C}_{2} \mathrm{D}_{2}^{+}\end{array}$ \\
\hline 14 & $\begin{array}{l}\mathrm{C}_{2} \mathrm{D}_{2}^{+} \\
\mathrm{CD}_{4}^{+} \\
\mathrm{C}_{2} \mathrm{D}_{4}^{+}\end{array}$ \\
\hline
\end{tabular}


Table II. Fragmentation Patterns ${ }^{\mathrm{a}}$

\begin{tabular}{|c|c|c|c|c|}
\hline $\mathbf{m} / \mathbf{e}$ & $\begin{array}{l}\text { Ethane-d } \\
C_{2} D_{6}\end{array}$ & $\begin{array}{l}\text { Ethylene- } \mathrm{d}_{4} \\
\mathrm{C}_{2} \mathrm{D}_{4}\end{array}$ & $\begin{array}{l}\text { Acetylene- } \\
d_{2} \\
C_{2} D_{2}\end{array}$ & $\begin{array}{l}\text { Tetradeute } \\
\text { ro- } \\
\text { methane } \\
\mathrm{CD}_{4}\end{array}$ \\
\hline 36 & 185 & & & \\
\hline 34 & 146 & & & \\
\hline 32 & 1000 & 1000 & & \\
\hline 30 & 273 & 618 & & \\
\hline 28 & 209 & 640 & 406 & \\
\hline 27 & & & 807 & \\
\hline 26 & & 106 & 1000 & \\
\hline 24 & & 32 & 286 & \\
\hline 20 & & & & 1000 \\
\hline 18 & & & & 830 \\
\hline 16 & 54 & 110 & 13 & 125 \\
\hline 14 & 24 & 64 & 136 & 72 \\
\hline 12 & & & 194 & \\
\hline 2 & & & & 30 \\
\hline
\end{tabular}

aData from Ref. 12. 


\section{Figure Captions}

Fig. 5-1 Time-of-flight data (OOOO) taken at $\theta_{\mathrm{i}}=30^{\circ}$ for (a) $\mathrm{m} / \mathrm{e}=20$ and (b) $\mathrm{m} / \mathrm{e}=18$. The fit $(-)$ is a simulated time-of-flight generated from the translational energy distribution in Fig. 2 for both (a) and (b).

Fig. 5-2 Translational energy distribution for tetradeuteromethane obtained by fitting $m / e=20$ data at all angles $\theta_{i}$ (some fits are shown in Fig. 3).

Fig. 5-3 Data (OOOO) taken for $\mathrm{m} / \mathrm{e}=20$ at different angles $\theta_{\mathrm{i}}$. The left side (a, c, and e) were measured with $\mathrm{T}_{\text {surf }}=570 \mathrm{~K}$. The right side ( $\mathrm{b}, \mathrm{d}, \mathrm{f}$ ) were measured with $T_{\text {surf }}=705 \mathrm{~K}$. The collisional ionization component is shown (......) in (c)-(f) (not a fit) and is off the scale of the graph. The simulated time-of-flight $(-)$ is generated from the translational energy distribution in Fig. 2. The same translational energy distribution is used to fit all the data $(a)-(f)$, for both surface temperatures.

Fig. 5-4 Data (OOOO) taken at $\mathrm{m} / \mathrm{e}=32$ for different angles $\theta_{\mathrm{i}}$ and $\mathrm{T}_{\text {surf }}=570$ K. The fit $(-)$ is a simulated time-of-flight spectrum generated from the translational energy distribution in Fig. 5.

Fig. 5-5 Translational energy distribution for ethylene- $\mathrm{d}_{4}$ obtained by fitting 
$\mathrm{m} / \mathrm{e}=32$ data at all angles $\theta_{\mathrm{i}}$ (some fits are shown in Fig. 4).

Fig. 5-6 Data (OOOO) taken at $\mathrm{m} / \mathrm{e}=26$ for different angles $\theta_{i}$ and two surface temperatures: $T_{\text {surf }}=570 \mathrm{~K}(\mathrm{a}, \mathrm{c}$, and $\mathrm{e})$ and $\mathrm{T}_{\text {surf }}=705 \mathrm{~K}(\mathrm{~b}, \mathrm{~d}$, and $f$ ). The collisional ionization component (off the scale of the graph) is shown in (c)-(f) (.....) (not a fit). The simulated time-of-flight spectra used to fit the product time-of-flight peaks are generated from the translational energy distributions of Fig. 5 (ethylene- $d_{4},-$ ) and Fig. 7 (acetylene- $\left.\mathrm{d}_{2},-\ldots\right)$. The total fit is also $\operatorname{shown}(\ldots)$ ).

Fig. 5-7 Translational energy distribution for acetylene- $\mathrm{d}_{2}$ obtained by fitting $\mathrm{m} / \mathrm{e}=26$ data at all angles $\theta_{\mathrm{i}}$ (some fits are shown in Fig. 6).

Fig. 5-8 Data (OOOO) taken at $\mathrm{m} / \mathrm{e}=16$ for different angles $\theta_{\mathrm{i}}$. The fit is generated from the translational energy distributions for ethylene- $d_{4}(-)$ (Fig. 5) and acetylene- $\mathrm{d}_{2}$ (----) (Fig. 7). $\mathrm{T}_{\text {surf }}=570 \mathrm{~K}$ for (a), (c), and (d) and $\mathrm{T}_{\text {surf }}=705 \mathrm{~K}$ for (b). In (d) the collisional ionization component (off the scale of the graph) is shown (......) (not a fit).

Fig. 5-9 Data (OOOO) taken at $\mathrm{m} / \mathrm{e}=14$ and $\theta_{\mathrm{i}}=25^{\circ}$ for $\mathrm{T}_{\text {surf }}=705 \mathrm{~K}$. The ethylene- $\mathrm{d}_{4}$ component (-----), the acetylene- $\mathrm{d}_{2}$ component $(-)$, and the 
tetradeuteromethane component $(---\ldots)$, which is negligible, are shown. The translational energy distributions of Figs. 2, 5, and 7 were used for tetradeuteromethane, ethylene- $\mathrm{d}_{4}$, and acetylene- $\mathrm{d}_{2}$, respectively, in fitting.

Fig. 5-10 Data (0000) taken for $\mathrm{m} / \mathrm{e}=30, \theta_{\mathrm{i}}=20^{\circ}$, and $\mathrm{T}_{\text {surf }}=570 \mathrm{~K}$. The fit $(-)$ is a simulated time-of-flight spectrum generated from the translational energy distribution in Fig. 5.

Fig. 5-11 The translational energy distributions of Figs. 2 (tetradeuteromethane, .....), 5 (ethylene- $\mathrm{d}_{4}, \ldots-\ldots$ ), and 7 (acetylene- $\mathrm{d}_{2}$ - . . ) are plotted with a Maxwell-Boltzmann distribution for $\mathrm{T}_{\text {surf }}=705$ $\mathrm{K}(-)$.

Fig. 5-12 The measured $\theta_{\mathrm{i}}$ distribution for acetylene- $\mathrm{d}_{2}(-)$ is plotted in polar form with a $\cos (\theta)$ distribution (•) for comparison. $T_{\text {surf }}=705 \mathrm{~K}$.

Fig. 5-13 The measured $\theta_{\mathrm{i}}$ distribution for ethylene- $\mathrm{d}_{4}(-)$ is plotted in polar form with a $\cos (\theta)$ distribution ( $)$ for comparison. $T_{\text {surf }}=705 \mathrm{~K}$. The data is best fit with a $\cos ^{2.6}(\theta)$ distribution, also shown ( 
Fig. 5-14 The measured $\theta_{\mathrm{i}}$ distribution for tetradeuteromethane ( $\left.\boldsymbol{\nabla}\right)$ is plotted in polar form with a $\cos (\theta)$ distribution (ब) for comparison. $\mathrm{T}_{\text {surf }}=705 \mathrm{~K}$. Part of the tetradeuteromethane product shows a "memory" of the beam by peaking near $\theta_{\mathrm{i}}=45^{\circ}$. 


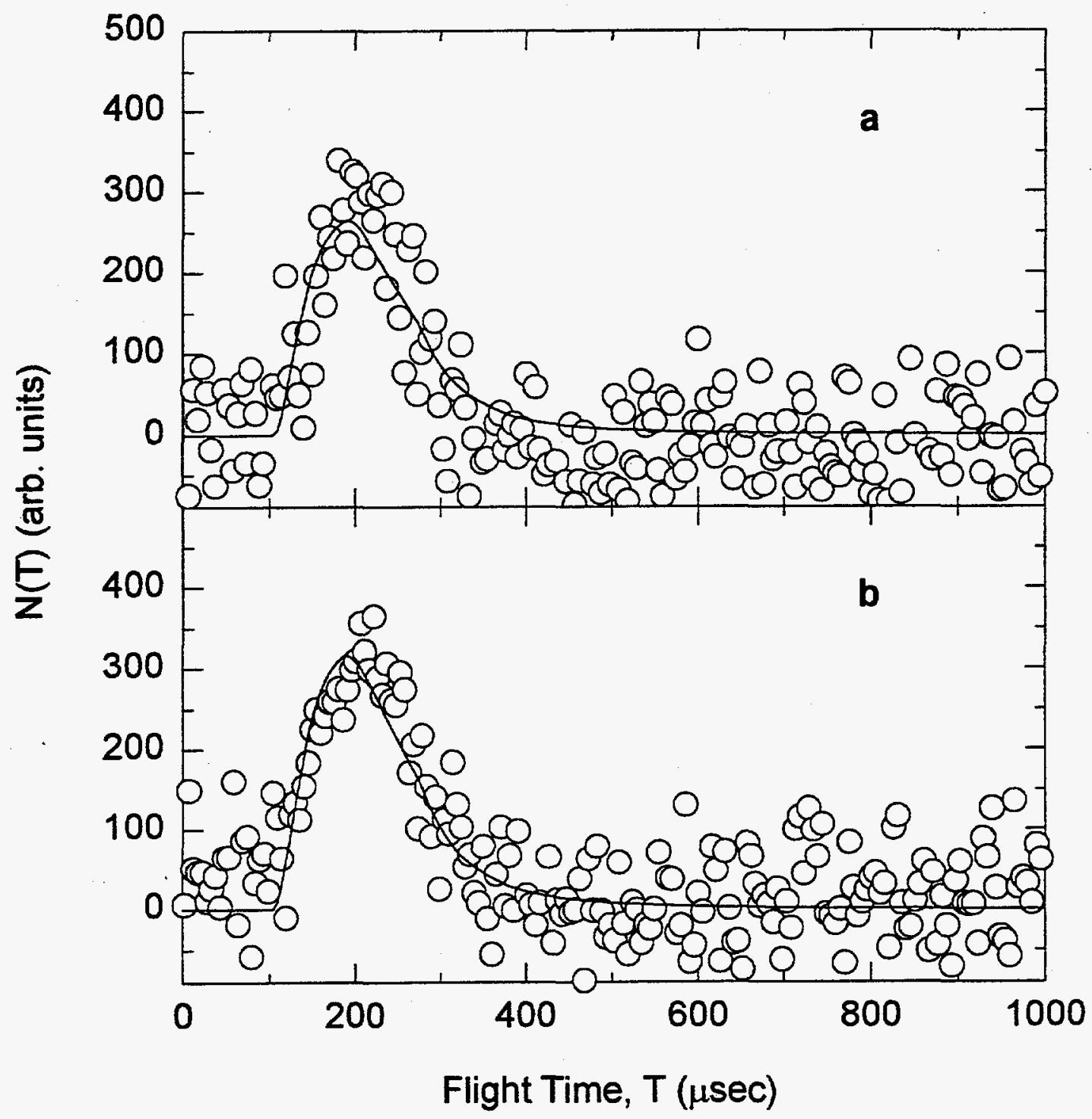

Figure 5-1 


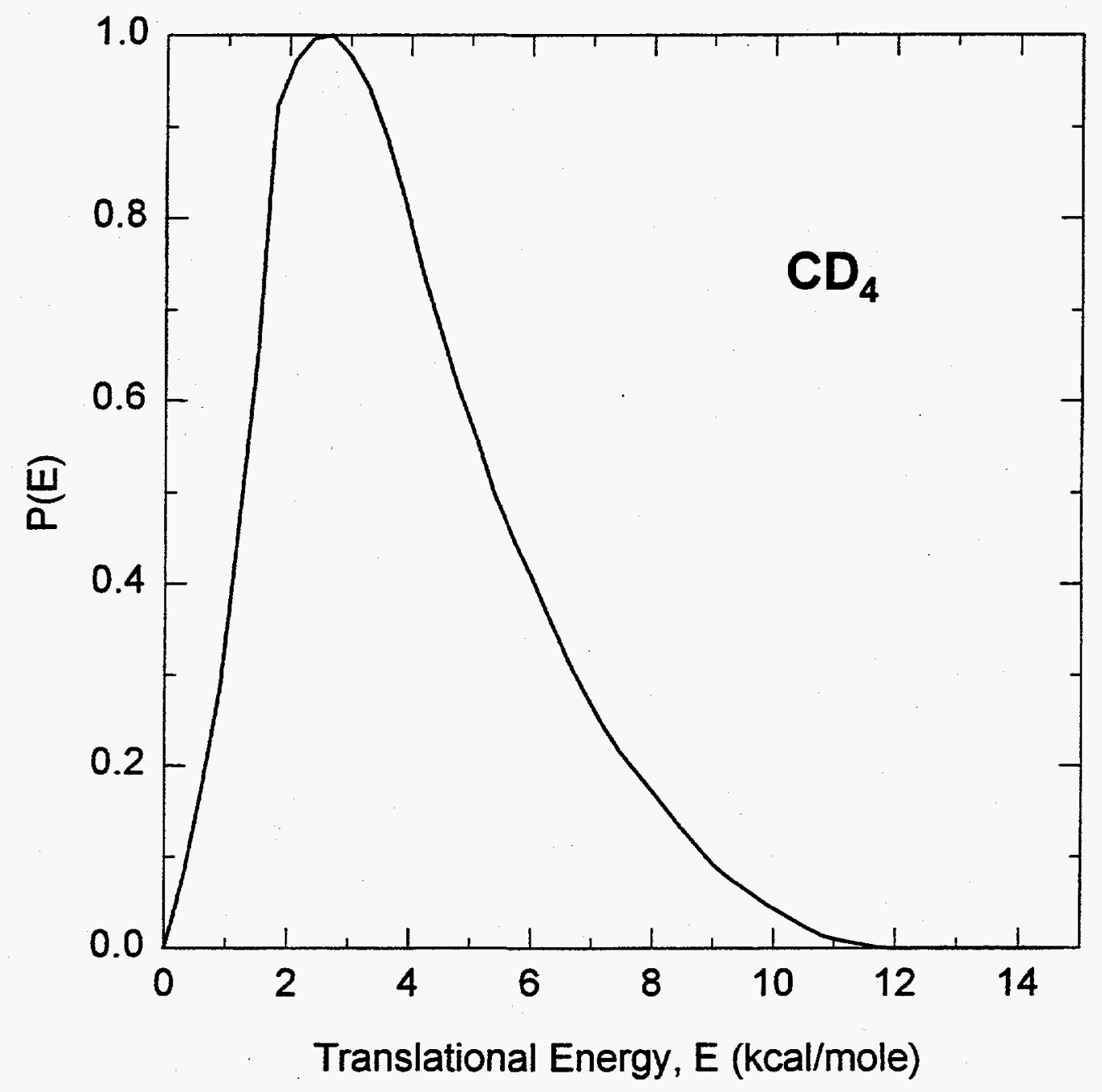

Figure 5-2 


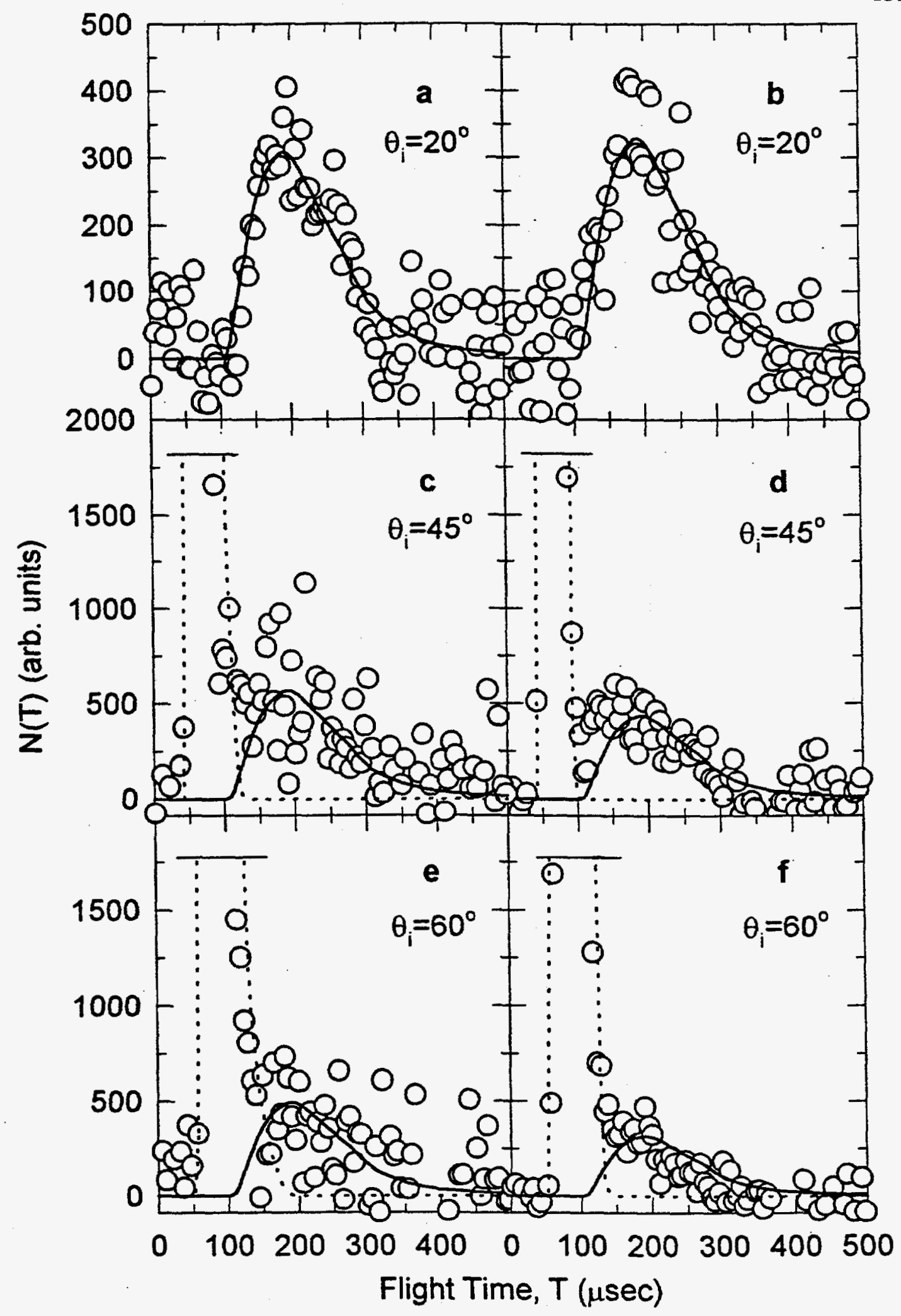

Figure 5-3 


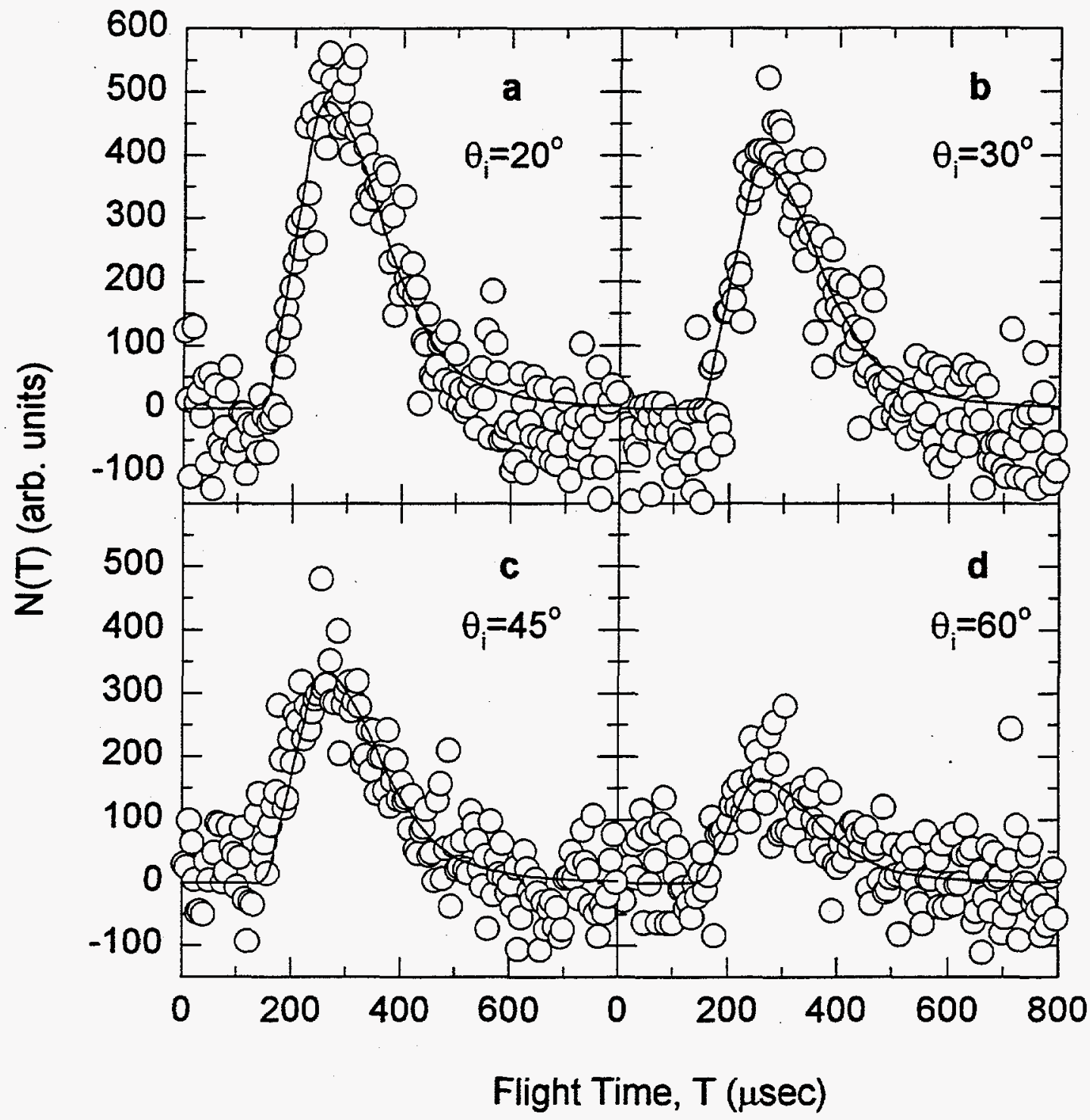

Figure 5-4 


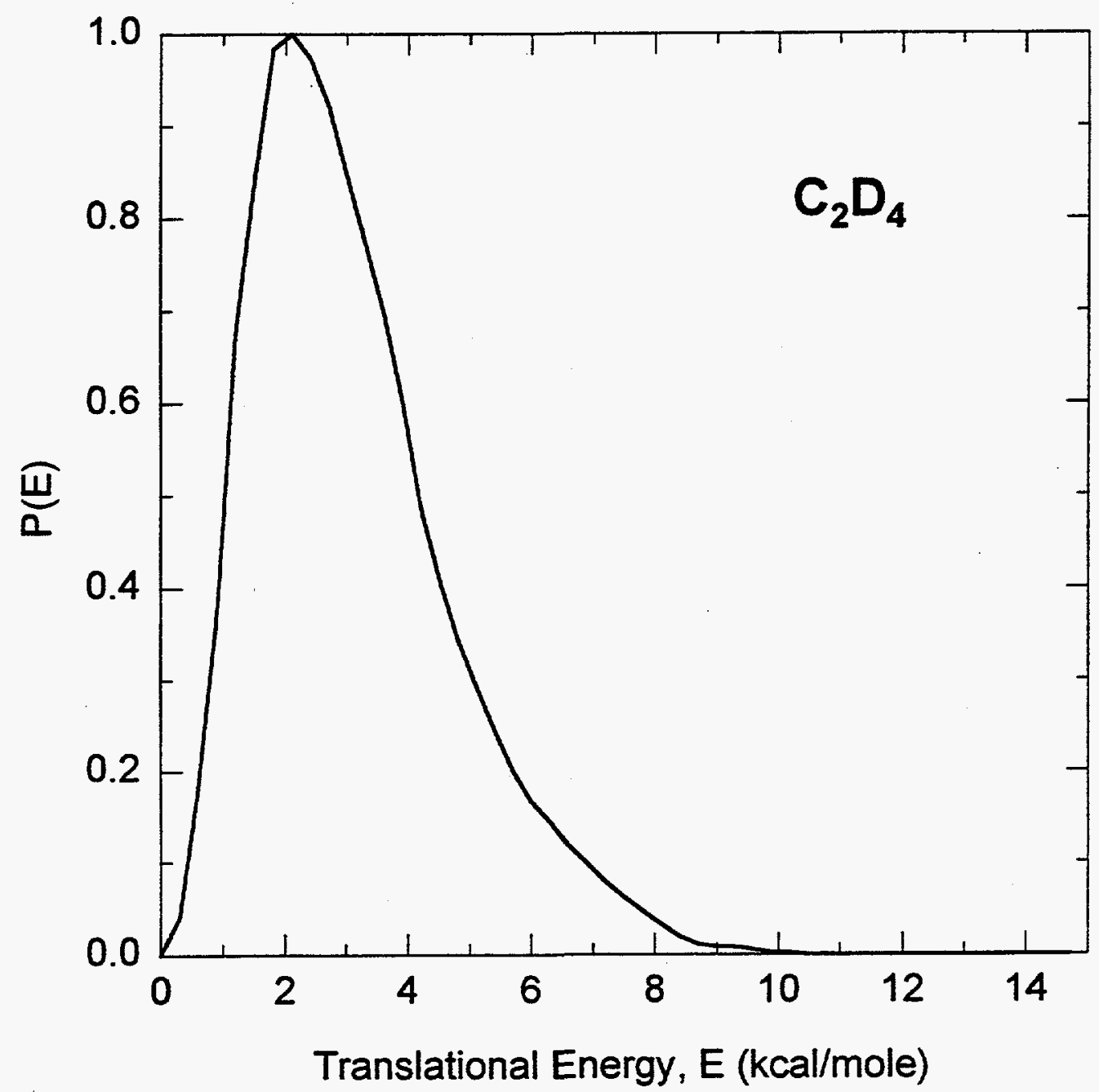

Figure 5-5 


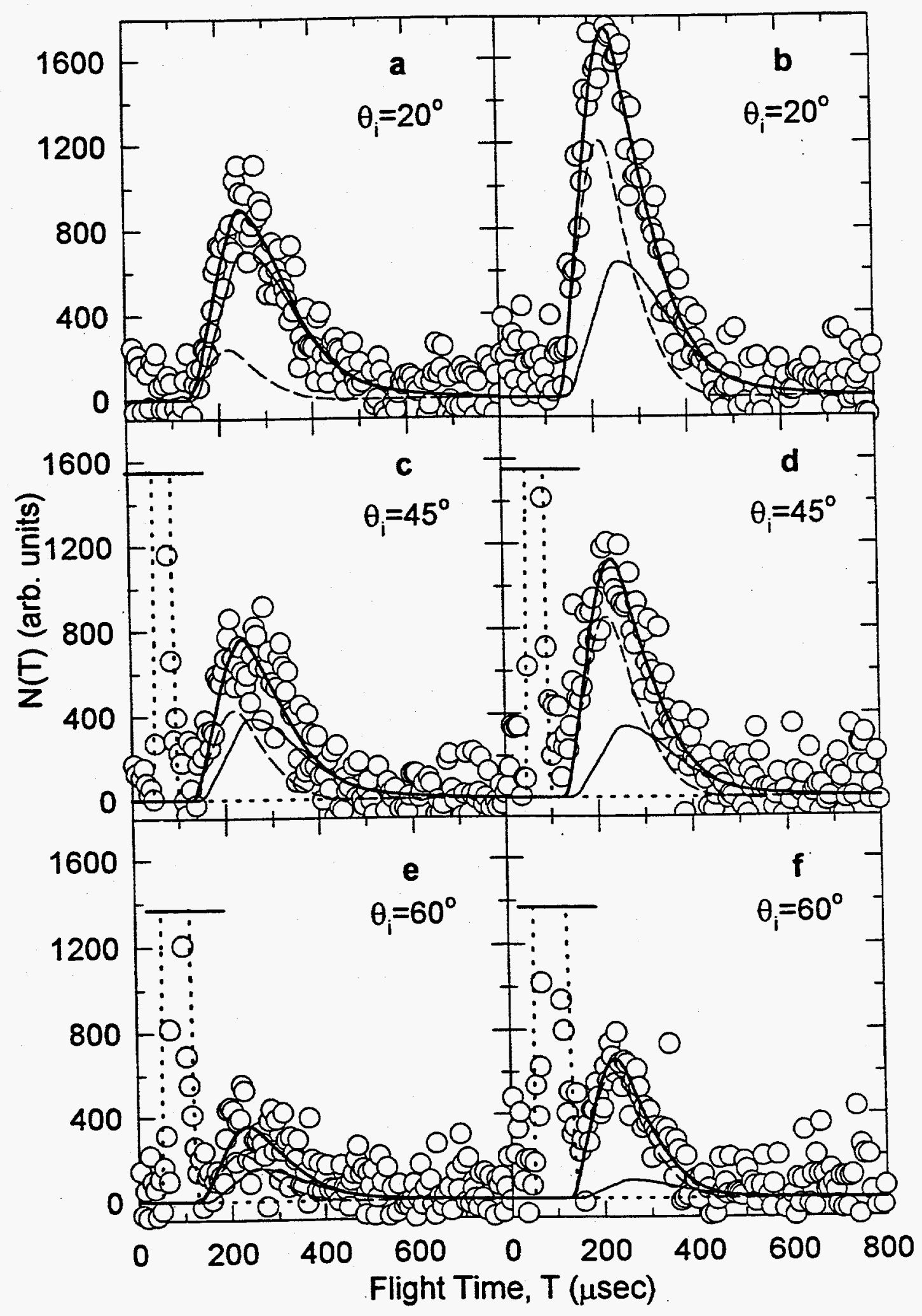

Figure 5-6 


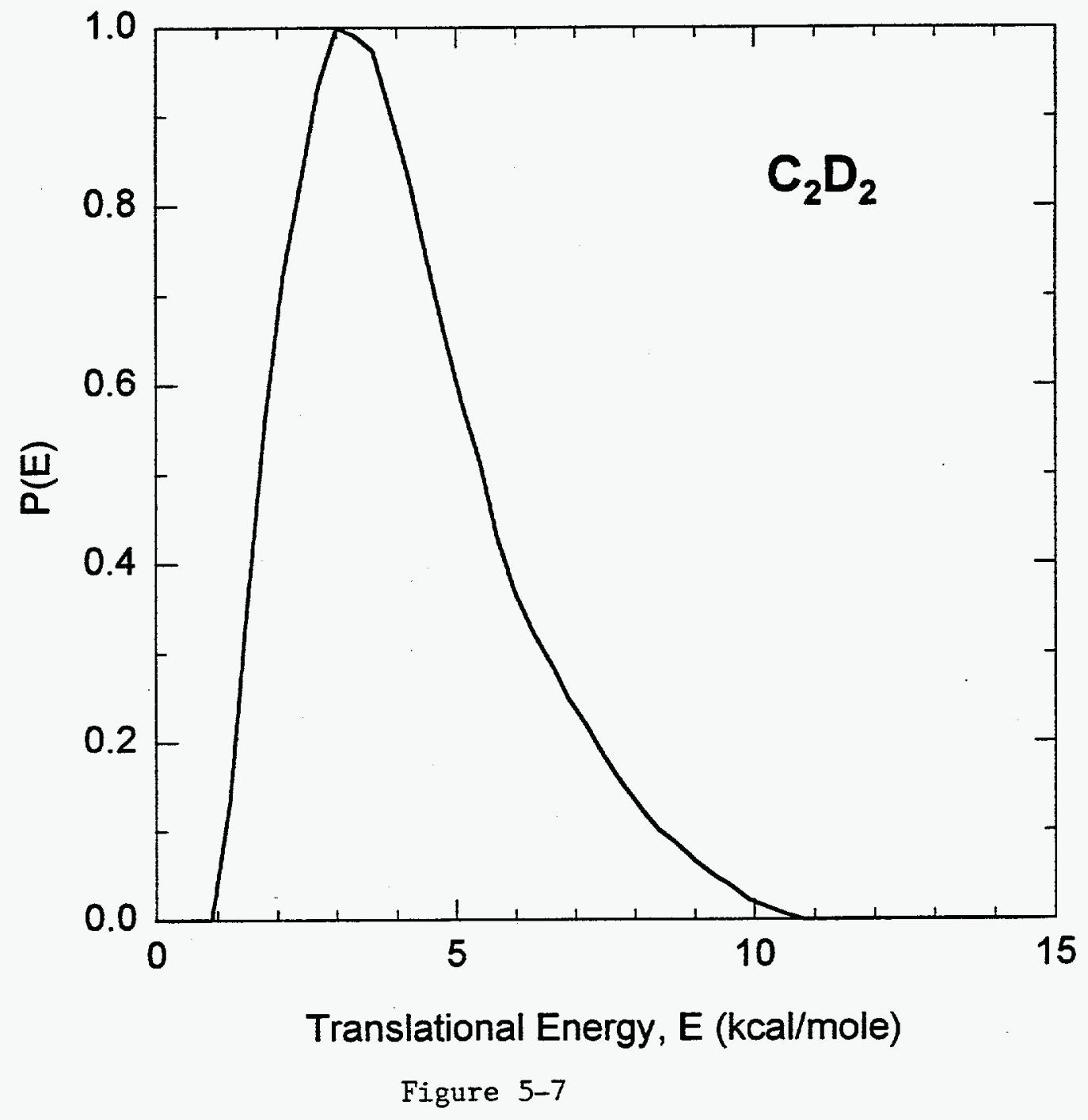




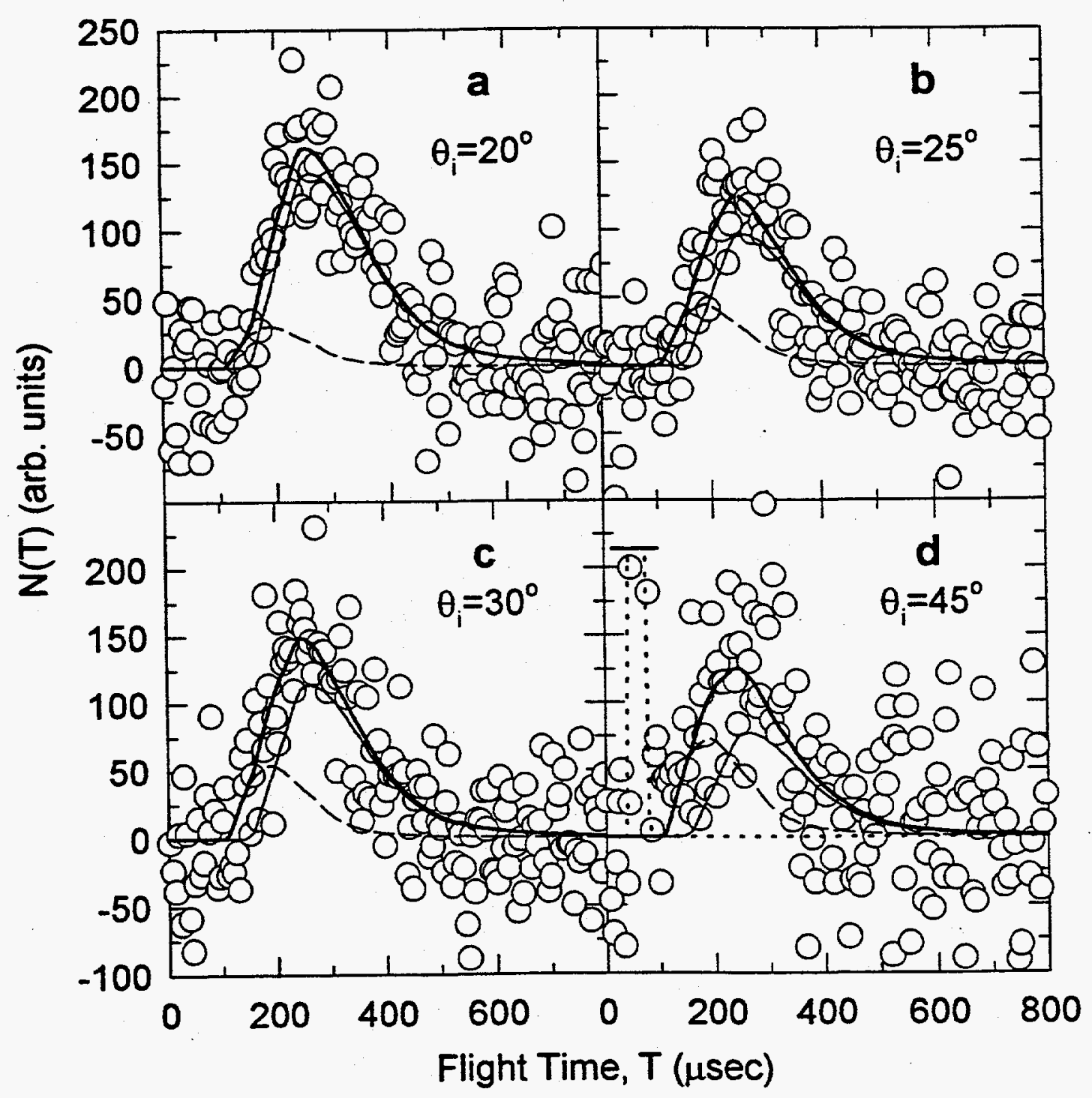

Figure 5-8 


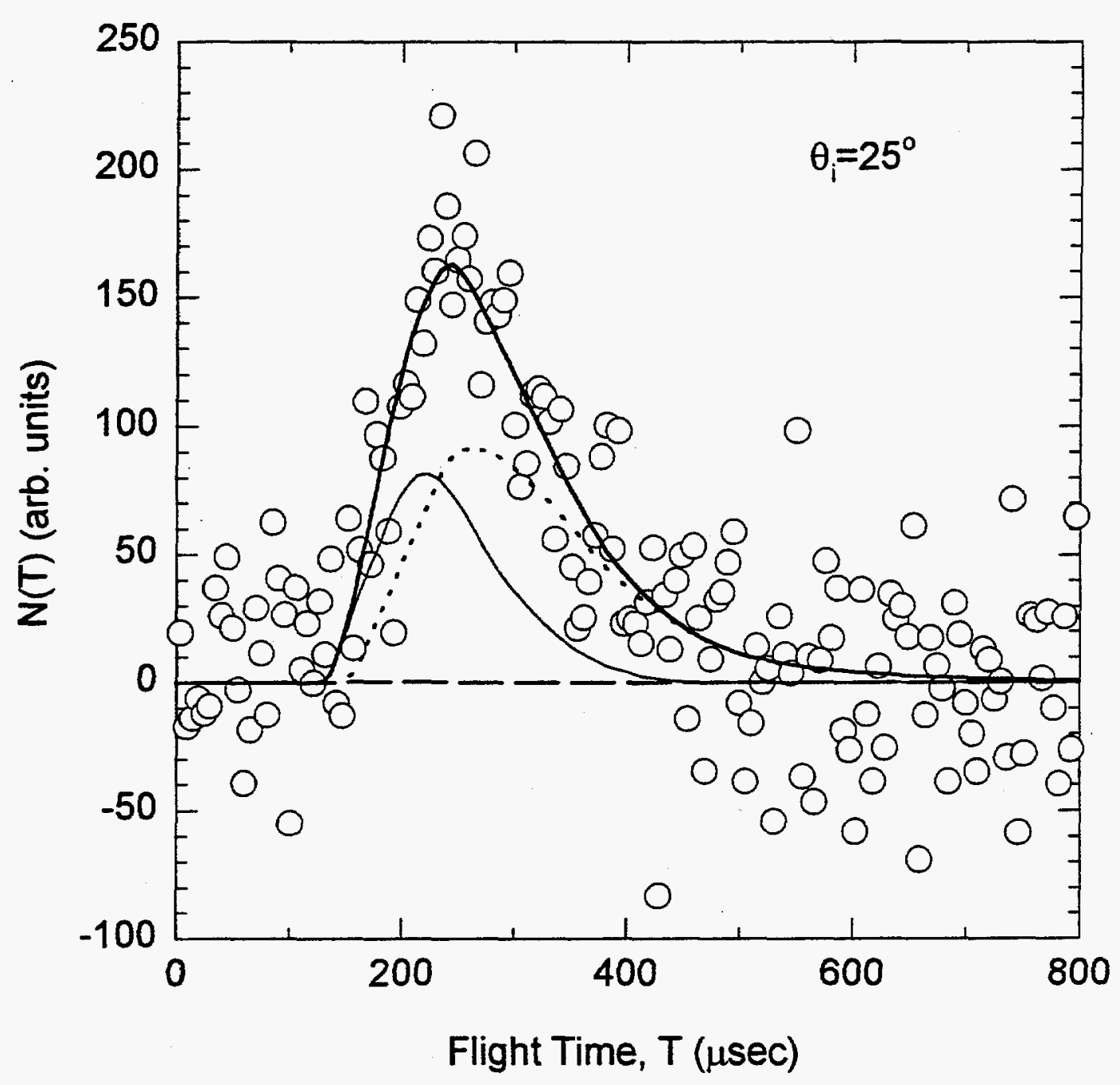

Figure 5-9 


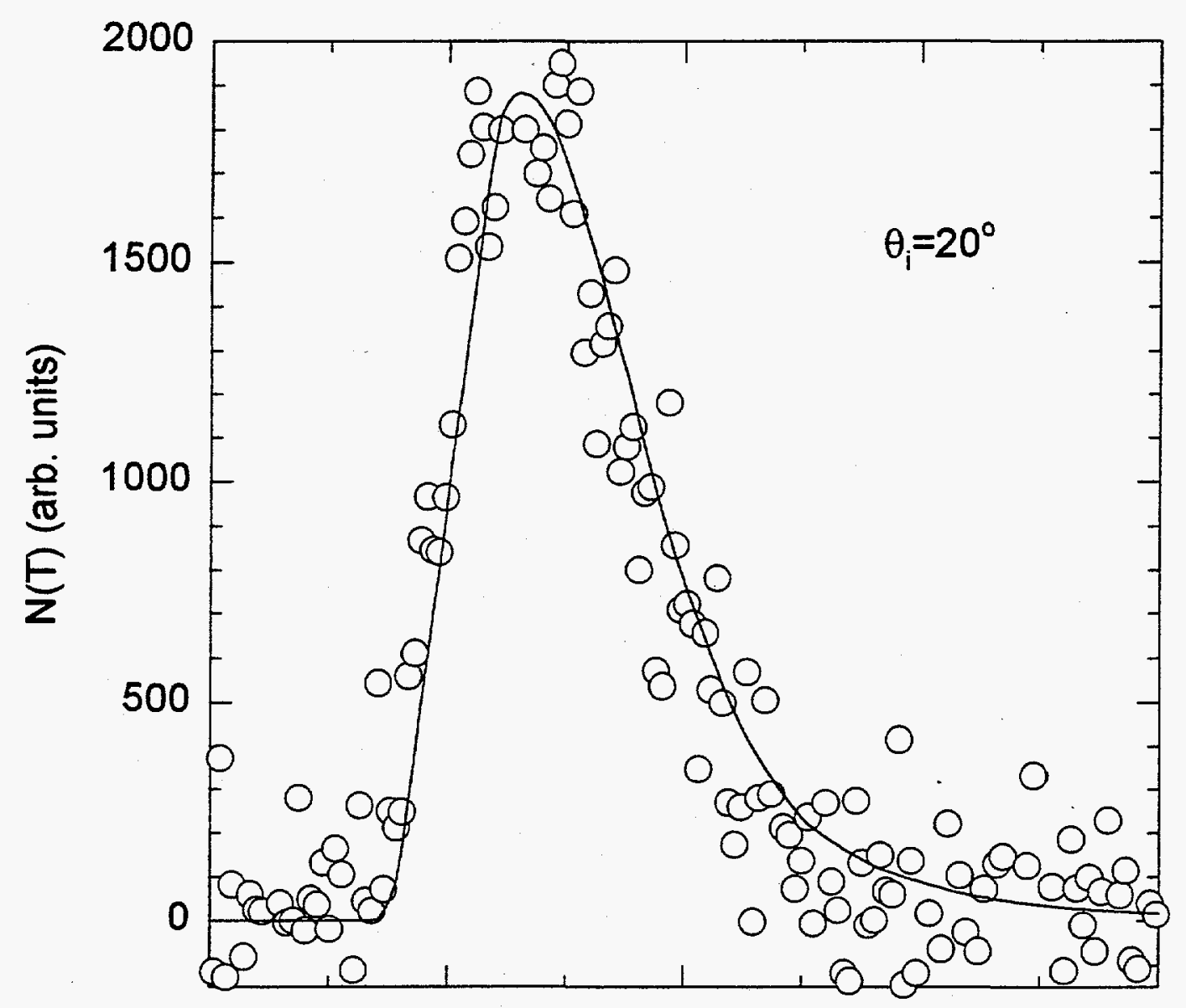

Flight Time, $\mathrm{T}$ ( $\mu \mathrm{sec}$ )

Figure 5-10 


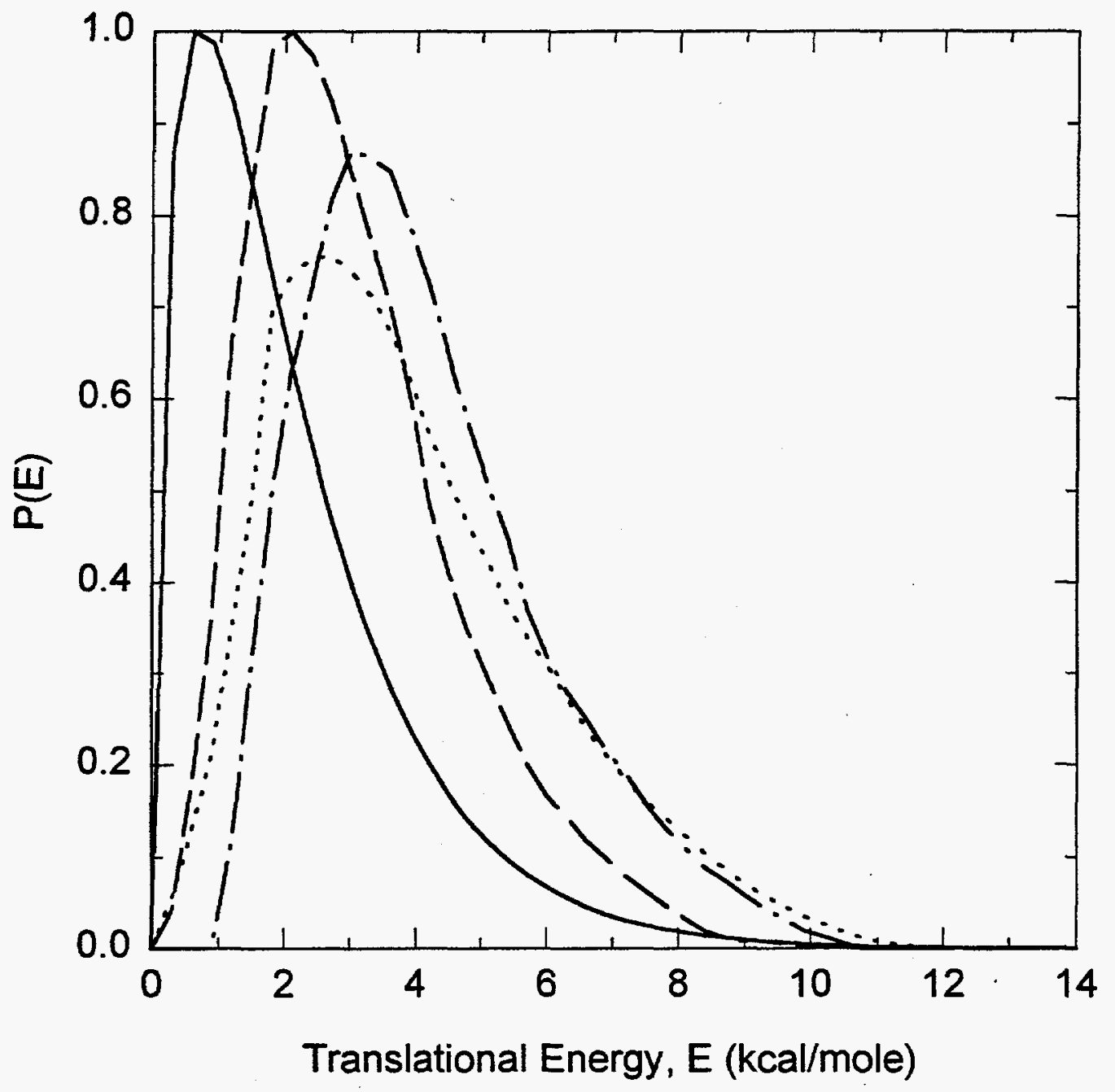

Figure 5-11 


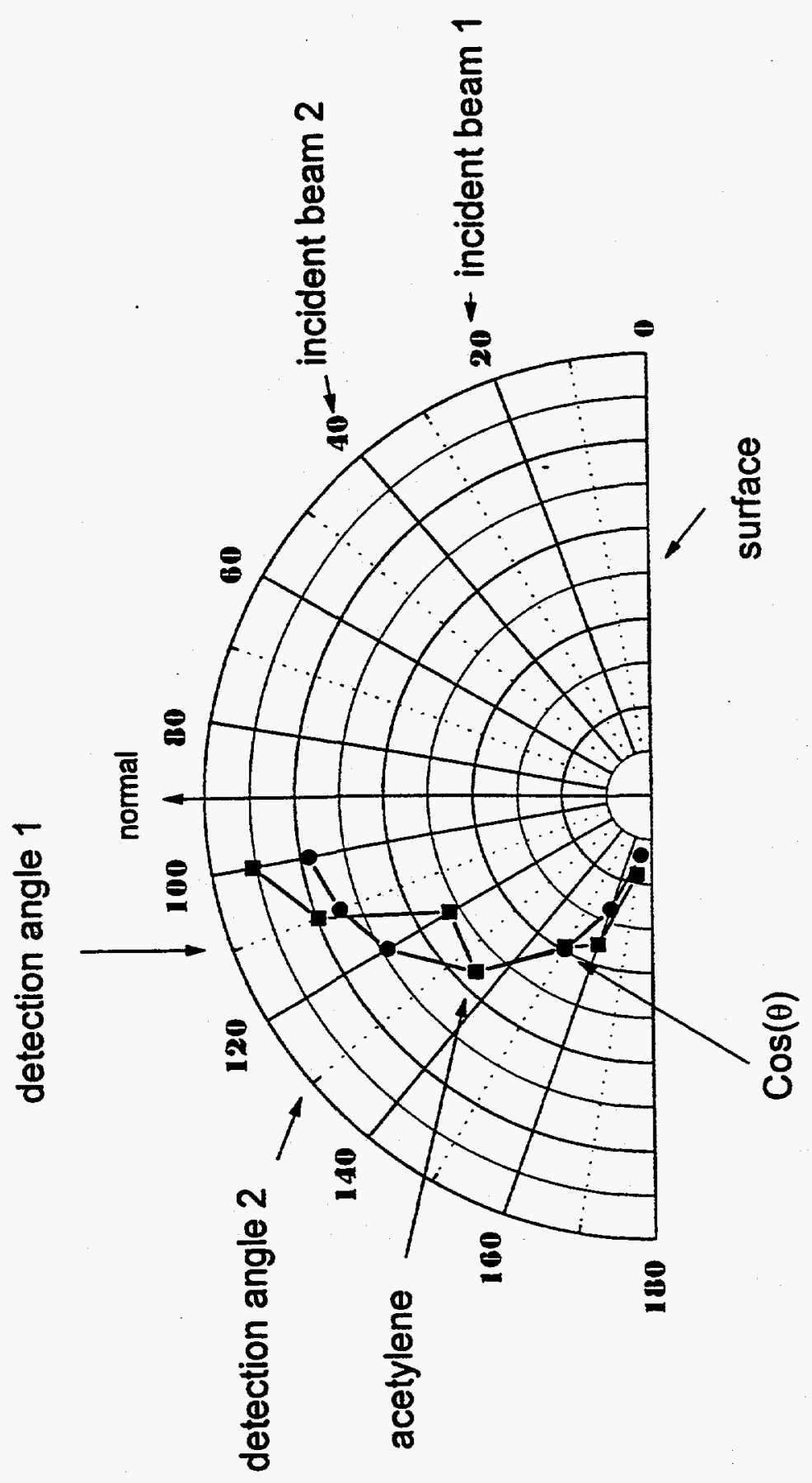

Figure 5-12 


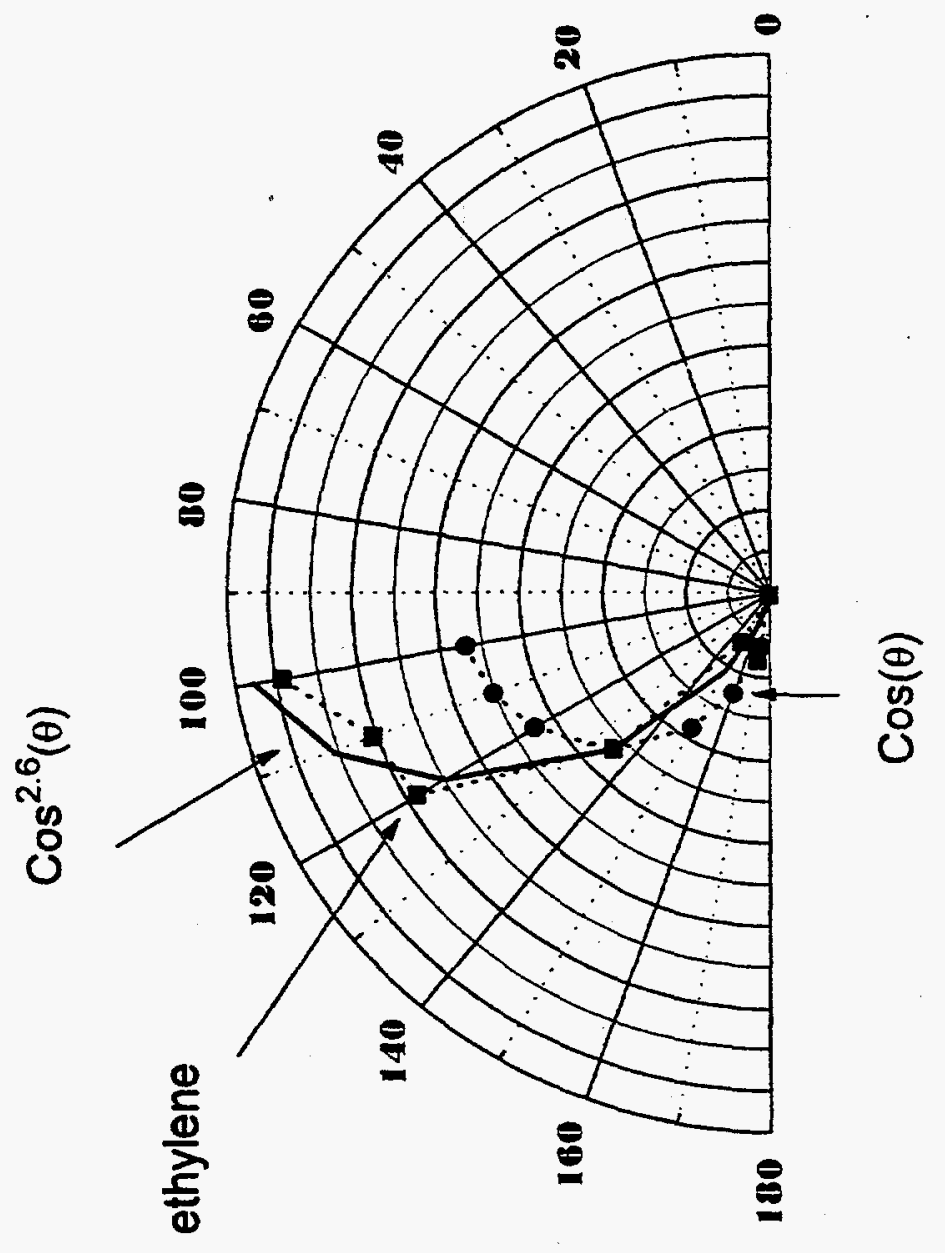

Figure 5-13 


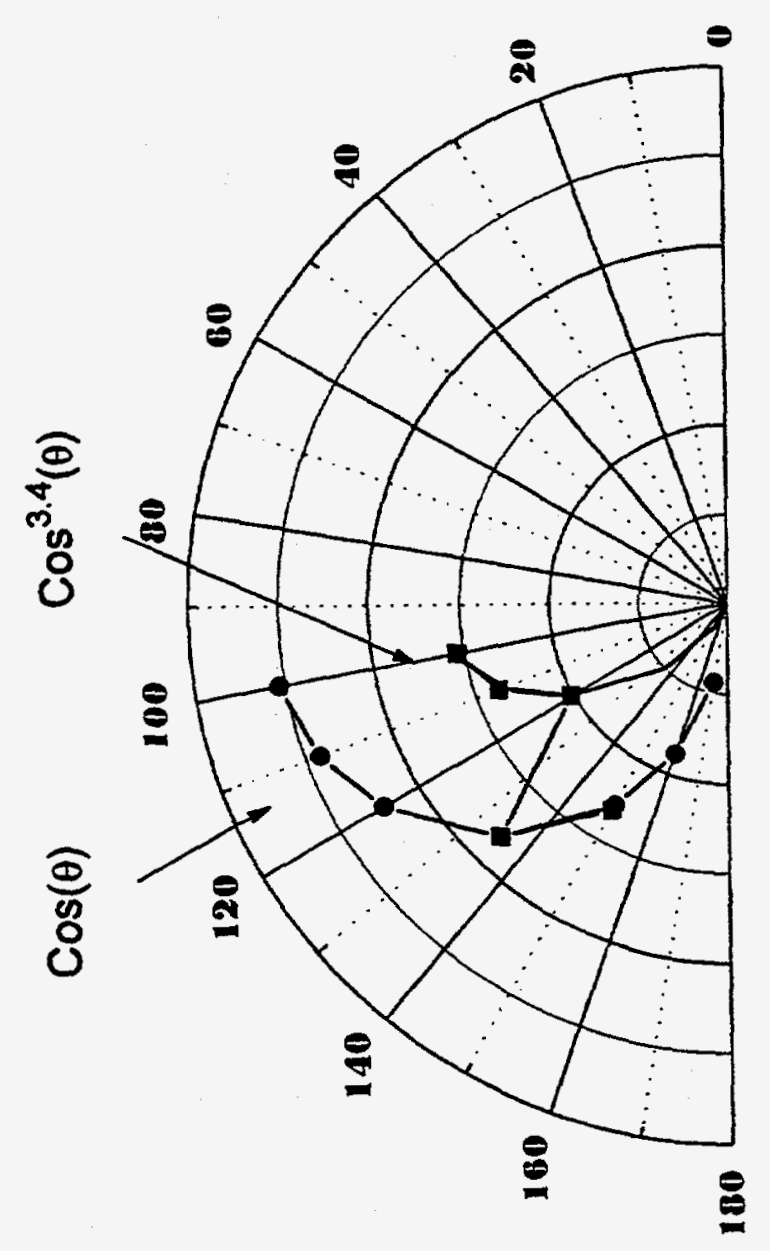

Figure 5-14 